University of Louisville

ThinkIR: The University of Louisville's Institutional Repository

Electronic Theses and Dissertations

8-2015

\title{
Synthesis, optimization, and degradation of a fish glue/PLGA polymer.
}

Adam T. Schworer 1989-

University of Louisville

Follow this and additional works at: https://ir.library.louisville.edu/etd

Part of the Biomedical Engineering and Bioengineering Commons

\section{Recommended Citation}

Schworer, Adam T. 1989-, "Synthesis, optimization, and degradation of a fish glue/PLGA polymer." (2015). Electronic Theses and Dissertations. Paper 2126.

https://doi.org/10.18297/etd/2126

This Master's Thesis is brought to you for free and open access by ThinkIR: The University of Louisville's Institutional Repository. It has been accepted for inclusion in Electronic Theses and Dissertations by an authorized administrator of ThinkIR: The University of Louisville's Institutional Repository. This title appears here courtesy of the author, who has retained all other copyrights. For more information, please contact thinkir@louisville.edu. 
Synthesis, Optimization, and Degradation of a Fish Glue/PLGA Polymer

By

Adam T. Schworer,

B.S., University of Louisville, 2011

\author{
A Thesis \\ Submitted to the Faculty of the \\ University of Louisville \\ J.B. Speed School of Engineering \\ as Partial Fulfillment of the Requirements \\ for the Professional Degree
}

\title{
MASTER OF ENGINEERING
}

Department of Bioengineering

July 2015 
Synthesis, Optimization, and Degradation of a Fish Glue/PLGA Polymer

Submitted By:

Adam Thomas Schworer

A Thesis Approved On

by the Following Reading and Examination Committee

Robert S. Keynton, PhD, Thesis Director

Martin G. O'Toole, $\mathrm{PhD}$

Robert W. Cohn, PhD 


\section{ACKNOWLEDGEMENTS}

The author would like to thank Dr. Scott Berry of the University of Wisconsin Department of Biomedical Engineering, the creator of the method for this study's application for the creation of this technology, Dr. Mark Crain, Scott Cambron, and Dr. Thomas Roussel of the University of Louisville Department of Bioengineering for their aid and wisdom, Devonnah Woodruff of the Department of Bioengineering for her input and insight, Nick Latino of the Department of Bioengineering for his hard work and dedication, Dr. Patricia Soucy of the Department of Bioengineering for her training help and instrumentation training, Dr. Martin O'Toole of the Department of Bioengineering for his help with experimental protocols and troubleshooting as well as service on this thesis committee, Dr. Robert Cohn of the Department of Electrical Engineering for serving on the Thesis Committee and his revisions to this work, Dr. Andrea Gobin for

her guidance in and outside of the classroom during all of my years at the University of Louisville, and lastly, Dr. Robert Keynton, Chairman of the Department of Bioengineering for serving as Thesis Advisor and for mentoring me throughout my undergraduate and graduate career. 


\begin{abstract}
Biomaterials used for tissue engineering, specifically polymers, come from either naturally occurring, like gelatin or collagen, or synthetic sources, such as polyesters. Often times, it is advantageous to combine biomaterials in order to maximize certain material properties, such as cellular adhesion and degradation. The purpose of this study is to provide direction for the synthesis, characterization, and a combination of a combination biomaterial between fish glue (FG), a natural source polymer, and polylactic-co-glycolic acid (PLGA), a synthetically made polyester. First, FG is characterized for molecular weight via Gel Permeation Chromatography, GPC. Two combination methods to mix FG/PLGA solutions are then compared. Finally, FG/PLGA mixtures of varying relative concentrations are subjected to degradation via treatment with phosphate buffered saline (PBS) and topographical analysis is performed. Degradation rate analysis showed that the samples tested lost approximately $70 \%$ of their mass after 12 hours of PBS treatment. Topographical analysis of FG/PLGA provided evidence indicating that in the later stages of degradation, as the FG is completely dissolved, small particles of PLGA remain. The work discussed in the proceeding document confirms the mixing of FG/PLGA, provides direction for an optimal mixing method and for characterization of FG, and documents a degradation and topographical analysis for small set of FG/PLGA mixes.
\end{abstract}




APPROVAL PAGE

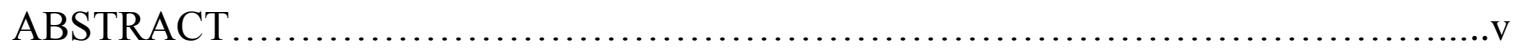

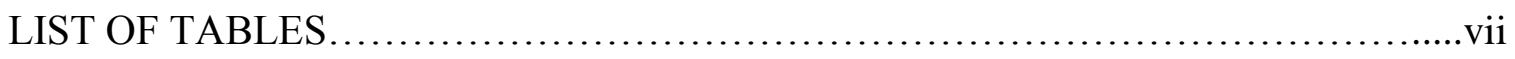

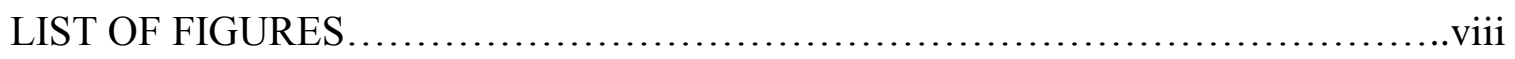

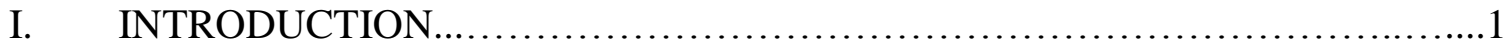

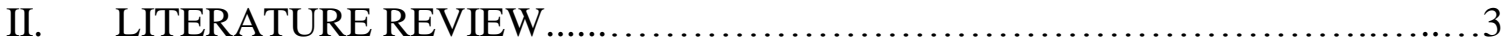

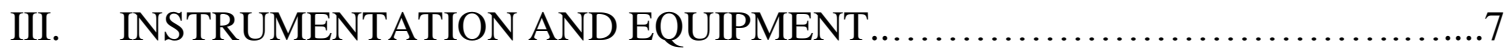

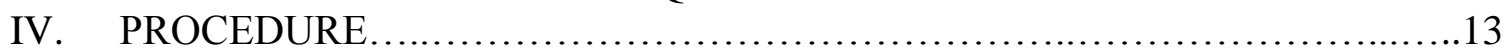

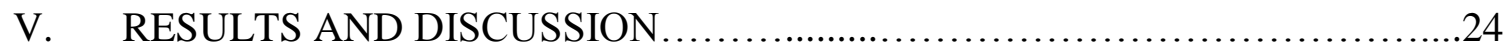

VI. CONCLUSIONS AND RECOMMENDATIONS …........................

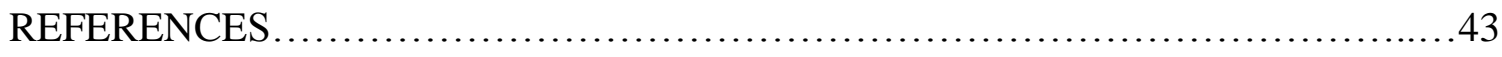

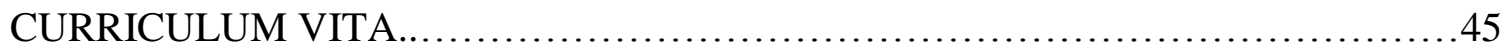

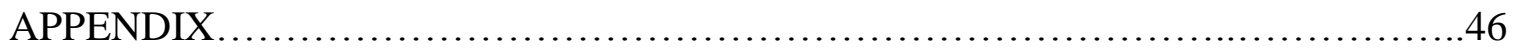




\section{LIST OF TABLES}

TABLE $1-$ Standard elution times and molecular weights........................ 30 


\section{LIST OF FIGURES}

FIGURE 1-Ultrasonic Cleaner used to indirectly mix solutions $\quad 8$

FIGURE 2-Heidolph Overhead stirrer used for mechanical mixing of solutions. $\quad 9$

FIGURE 3-Spectrophotometer used for absorbance measurements. 10

FIGURE 4-Laurell Technologies spin coater used for cover slide coating. 11

FIGURE 5-Probability plot of Heated and No Heat \% Solid Conc. 25

FIGURE 6-Mann-Whitney Test of Heated vs No Heat \% Solid Conc. 25

FIGURE 7-Fish glue refractive index spectra. 28

FIGURE 8-Fish glue 280 nm spectra.

FIGURE 9-All sample RI spectra.

FIGURE 10-All sample $280 \mathrm{~nm}$ spectra. 30

FIGURE 11-Standard Curve of RI elution time and MW. 31

FIGURE 12-Standard Curve of $280 \mathrm{~nm}$ elution time and MW. 31

FIGURE 13-Absorbance spectra of FG, PLGA, 4 hr Sonication, 4 hr Mechanical. 34

FIGURE 14-Absorbance spectra of timed mechanical mixes and $4 \mathrm{hr}$ Sonication. 34

FIGURE 15-Two Sample t-test of Absorbance at 260 of mixing types 35

FIGURE 16-- Two Sample t-test of Absorbance at 280 of mixing types 35

FIGURE 17- Two Sample t-test of Absorbance at 260:280 of mixing types 36

FIGURE 18-Mean Absorbances at $280 \mathrm{~nm}$ with standard deviation bars 36

FIGURE 19-Mean Absorbance at $260 \mathrm{~nm}$ with standard deviation bars 37

FIGURE 20-Percent Weight remaining by days of different concentrated mixtures. 38

FIGURE 21—SEM image of 65/35 Time point 5

FIGURE 22-SEM image of 65/35 Time point 6 


\section{INTRODUCTION}

\subsection{Definition of the Problem}

A major area of concern in Tissue Engineering (TE) involves material selection; specifically, it is necessary to select a material which will provide a substrate for cellular attachment and proliferation and degrade in a desirable timeframe. The focus of the work presented in this study is to develop a material that will degrade but maintain structural integrity for 3-14 days to enable deposition and proliferation of capillary cells to form functional 3D capillaries. The problem to be addressed by this thesis work is to identify the optimal mixing method to combine PLGA and Fish Glue, to characterize fish glue as a material used in TE as its main area of use is by artists, to identify the degradation rate of a PLGA/Fish Glue polymer in an environment similar to that of the human body, and to topographically visualize the physical features of a PLGA/Fish Glue polymer while it is subjected to a physiologically analogous environment.

\subsection{Brief History}

Biomaterials used for TE can come from various sources but can be grouped into two categories, synthetic or naturally occurring. In the field of TE, capillary synthesis is dependent upon using a proper biomaterial as a viable tissue scaffold. Naturally occurring materials, such as collagen, gelatin, or silk, can degrade too quickly or are too cumbersome to manipulate in a repeatable fashion. Synthetic materials like poly lactic acid (PLA), poly glycolic acid (PGA), poly co-lactic glycolic acid (PLGA) degrade too 
slowly and can cause adverse reactions from the native tissues. Therefore, a material which combines the cellular growth benefits of a naturally occurring polymer, like gelatin or FG, and the repeatable processing and manipulation ability of PLGA would be advantageous for capillary synthesis.

\subsection{Purpose of Research}

The primary purpose of this research is to characterize the degradation of differing concentrations of PLGA and Fish Glue in a physiologically analogous environment; effectively, characterizing the hydrolysis rate of different relative concentrations of the two polymers in question. The secondary purpose was to determine the best mixing method for FG and PLGA. 


\section{LITERATURE REVIEW}

\subsection{Overview}

In the field of TE, capillary synthesis, specifically the ability to create a 3D network of microvascular tissue, is necessary for organ synthesis due to the need for nutrient, gas, and waste exchange provided by a capillary bed. In order to create a complex capillary system, a cellular scaffold must be used to support the capillary cell growth and proliferation. Such scaffolds can be comprised of many various synthetic, such as PLA, PGA, PLGA, or biologically derived materials, like collagen, gelatin, chitosan, or silk. The following section contains a brief literature review of research related to Fish glue, a gelatinous biologically derived material, and PLGA, a commonly used synthetic polymer in TE. Finally, multiple approaches to mixing polymers are outlined as well as their efficacy.

\subsubsection{Fish Glue}

Fish glue (FG) is a natural polymer commonly used as an adhesive made by the heating of fish scales and bones and other various parts in water until dissolution, this heating can be performed at atmospheric pressure or at an elevated temperature to speed up the process. FG has been used as a material by artists for millennia because it is inexpensive and possesses the ability to be re-worked (Petukhova 2000). Even after a bond is made, FG maintains the property of being water soluble enabling an artist to easily make changes through wetting. In the field of Food Science, FG is a commonly used material; 
therefore, many characteristics and properties of fish gelatins have been analyzed including amino acid content and its dependence on fish species (Carbini et al. 1996). Even though FG is an established tool for artists, its use as a biomaterial has not been published to date. Fish gelatin has been shown to have a lower amount of hydroxyproline and proline imino acids than mammalian gelatins; moreover, colder water fish species proved to have the lower imino acid content than warmer water fish species (Gudmundsson 2002). Degradation of the collagen present in the fish scales and bones occurs during this heating process, the extent of degradation is generally unknown. Carbini et al. a curie-point pyrolysis-gas chromatography/mass spectroscopy study of fish glue versus other proteinaceous binders: milk casein, egg yolk, egg albumin, bone glue, skin glue, and rabbit glue (Carbini et al. 1996). Amino acid analysis, SDS-PAGE electrophoresis, and FTIR spectroscopy has been used as a means of analysis to determine the makeup of extracted fish collagen(J.H. Muyonga, Cole, and Duodu 2004)(Zhang, Xu, and Wang 2011). FTIR spectrum were compared between gelatin and collagen extracted from fish(J.H Muyonga, Cole, and Duodu 2004). Varying species of fish gelatin were compared through molecular weight determination via HPLC/GPC, isoelectric point determination, SDS-PAGE electrophoresis, viscosity, circular dichroism, and rheological measurements (Gudmundsson 2002)(Turnay and Ferna 2002). However, a comparative study of fish glue to both gelatin and collagen has not been performed. 


\subsubsection{Poly co-lactic glycolic acid}

PLGA is a polyester synthesized through the polymerization of glycolic acid and lactic acid monomers. PLGA has many uses and applications as a biomaterial: drug delivery in pharmaceuticals, bone grafts, sutures, and as cellular scaffolds. The degradation rates of PGA, PLA, and PLGA have been highly studied; furthermore, studies have shown that the degradation time of PLGA changes with the relative ratio of lactic to glycolic acid monomers. The higher the ratio of lactic acid to glycolic acid then degradation time is longer. This phenomena is due to the fact that PGA is highly crystalline and has a faster degradation rate as compared to PLA, 2 months versus 12 months. (Park 1995) (Ulery, Nair, and Laurencin 2011) (Makadia and Siegel 2011) (Grayson et al. 2004)

\subsubsection{Polymer mixing methods}

Two groups discuss success in mixing fish gelatin and PLLA dissolved in $88 \%$ formic acid or $90 \%$ acetic acid in distilled water and dichloromethane, respectively, through gently stirring gelatin (An 2010) (Gu 2009). With the use of aqueous solvents, collagen and poly acrylic acid have been shown create complexes by means of shaking followed by centrifuging (Barbani 1999). Composite films of collagen and poly( $\varepsilon$-caprolactone) have been fabricated by first treating freeze-dried collagen with PCL dissolved in dichloromethane then allowing the solvent to evaporate (Coombes 2002). Gu et al. outline the creation of a PLGA/gelatin composite through first dissolving PLGA in chloroform, then dispersing gelatin microparticles into the solution via ultrasonic wave energy, chloroform was then completely removed via evaporation followed by vacuum drying. The resulting scaffold was then treated with water dissolved gelatin; finally, the 
scaffold was twice cross-linked via treatment with 1-ethyl-3-(3-dimethyl-amino- propyl)carbodiimide (EDAC) and freeze-dried (Gu 2009). PLGA and collagen, of an unknown source, have been dissolved in hexafluoroisopropanol and successfully mixed with magnetic stir bar (Jose 2009). PLGA, of an unknown relative ratio, and gelatin, from an unknown source, have been reported to be mixed through dissolution of both materials in dichloromethane, treatment with an ultrasonic wave, followed by solvent evaporation ( $\mathrm{Li}$ 2007). Li et al. make no mention or measurement of mixing; however, they do present degradation profiles of differing relative ratios as well as SEM images to illustrate the microstructure and level of mixing. No previous research has been found that illustrates the successful mixing of FG and PLGA. 


\section{INSTRUMENTATION AND EQUIPMENT}

The following section identifies the equipment and instrumentation used for this study. In addition, a description of each piece of equipment and instrumentation is provided. A description of how each piece of equipment and the instrumentation were implemented in the study is also provided.

\subsection{Sonication Instrumentation}

The instrument used for ultrasonic mixing for this study was a FS60 Ultrasonic Cleaner (Thermo Fisher Scientific, Inc., Waltham, MA; FIGURE 1). This ultrasonic cleaner consists of a 6 quart stainless steel tank which is bonded to a piezoelectric transducer. To operate, the tank was filled with water until the water level was 1 inch from the top of the

tank. A glass vial filled with the desired amount of materials to be mixed was placed in a plastic holding tray, which floated on the top of the water. The cleaner was activated by turning the timing knob clockwise to 60 minutes. When the cleaner is activated, the piezoelectric transducer vibrates at a frequency of $40 \mathrm{kHz}$ causing air bubbles to form, grow, and collapse within the water in the tank, which indirectly mixed solutions in the glass vial. 


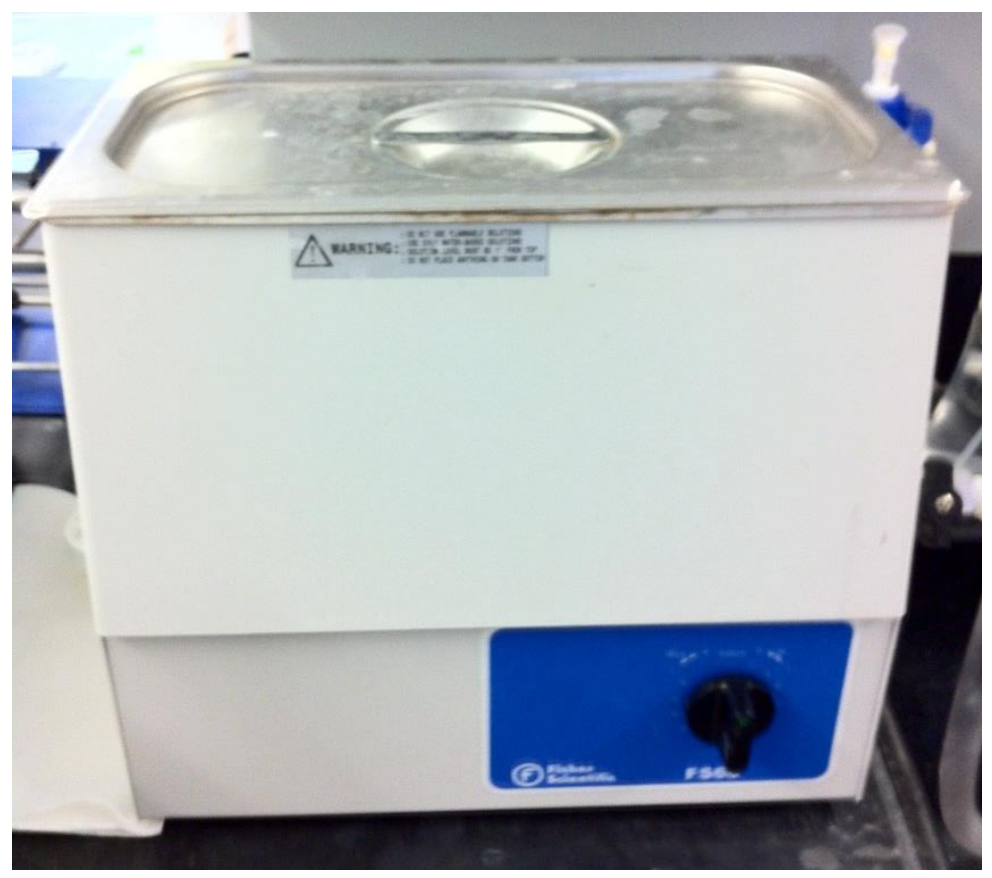

FIGURE 1-Ultrasonic Cleaner used to indirectly mix solutions

3.2 Mechanical Mixing Instrumentation 
An overhead stirrer (RZR 2021, Heidolph Instruments, Schwabach, Germany;

FIGURE 2) was used to directly mechanically mix the solutions. The overhead stirrer allowed the user to change the stirring rate, in rotations per minute, to a desired setting and operated continuously for a desired amount of time. To set up, a steel wire, approximately $2.5 \mathrm{~cm}$ in length with a bend of 15 degrees in the middle of the wire, was fastened in the Jacob's chuck of the overhead stirrer. Before mixing, the stirrer was turned on and the mixing speed knob was toggled until the desired mixing rate of 100 rpm appeared in the LCD display. A vial containing the desired solution to be mixed was positioned below the stirrer so that the bottom end of the steel wire was approximately $1 \mathrm{~mm}$ from the bottom of the vial. 


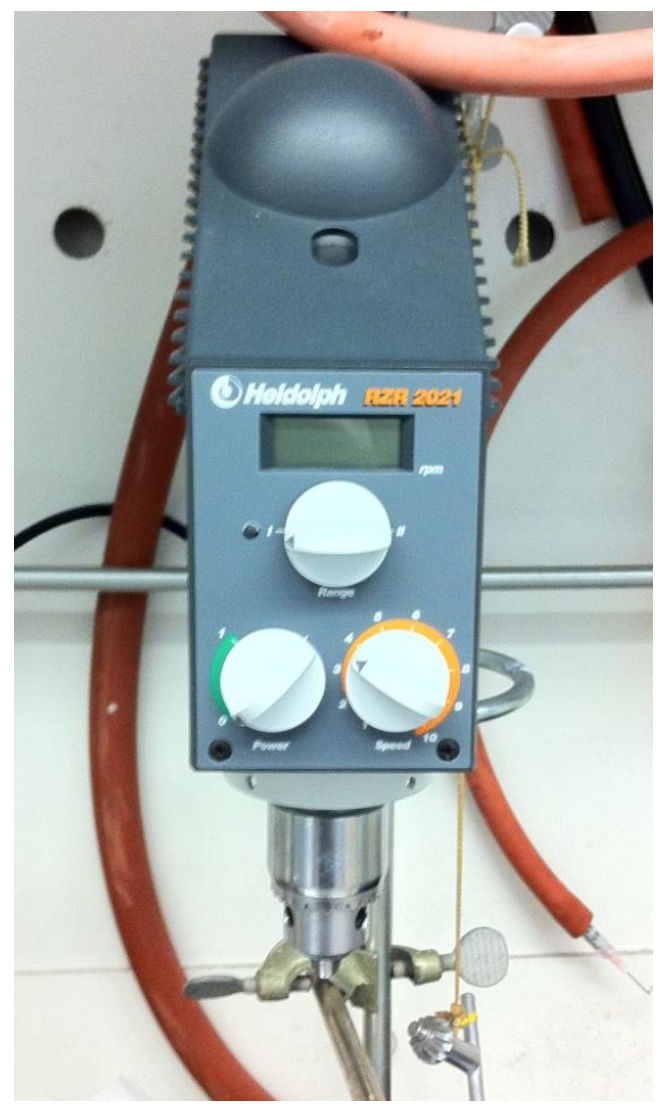

FIGURE 2-Heidolph overhead stirrer used for mechanical mixing of solutions.

\subsection{UV-VIS Spectrophotometer}

A UV/Vis Spectrophotometer (DU 530 Life Science, Beckman Coulter Corp., Brea, CA;

FIGURE 3) was used in this study to measure absorbance of light at varying wavelengths through samples. Briefly, the spectrophotometer operates by emitting a precise wavelength of light onto a sample cuvette and measures the amount of light which passes through the sample. Absorbance is then calculated by the spectrophotometer by performing a base ten logarithm of the quotient of the incident and transmitted light intensities, respectively. To operate, the system was blanked by placing a cuvette of distilled water in the specimen holder and pressing the blank command button on the system. Each sample used for absorbance analysis was diluted to a $1 \%$ concentration in distilled water. An absorbance spectra was obtained for each sample between the 
wavelengths of 190 nanometers and 350 nanometers using the absorbance sweep function of the spectrophotometer with a 1 nanometer step setting.

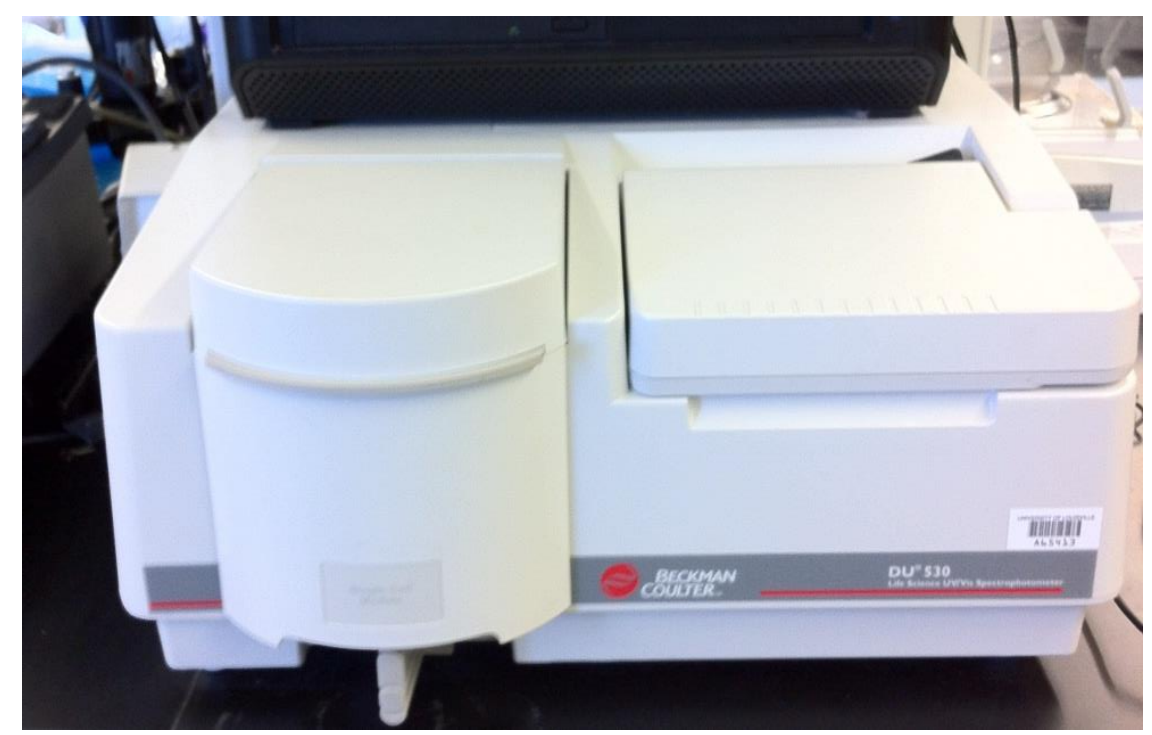

FIGURE 3-Spectrophotometer used for absorbance measurements.

\subsection{Spin Coating Instrumentation}

A spin coater (Model WS-400B-6NPP/LITE, Laurell Technologies Corporation, North Wales, PA; FIGURE 4) was utilized for depositing a thin layer of polymer solution onto a glass cover slide. Briefly, a spin coater works by rotating a substrate, that has been deposited with an excess amount of liquid material, at a high speed causing the material to spread evenly across the substrate due to centrifugal force. For this study, spin coated samples were prepared by depositing a $25 \mu \mathrm{L}$ volume of the desired polymer mixture onto the center of a glass microscope cover slide (12-5545-80 12 CIR.-1 Glass Microscope slide, Thermo Fisher Scientific, Waltham, MA). Immediately after the polymer has been deposited, the glass microscope cover slide was rotated at a speed of $3000 \mathrm{rpm}$ for 15 seconds. 


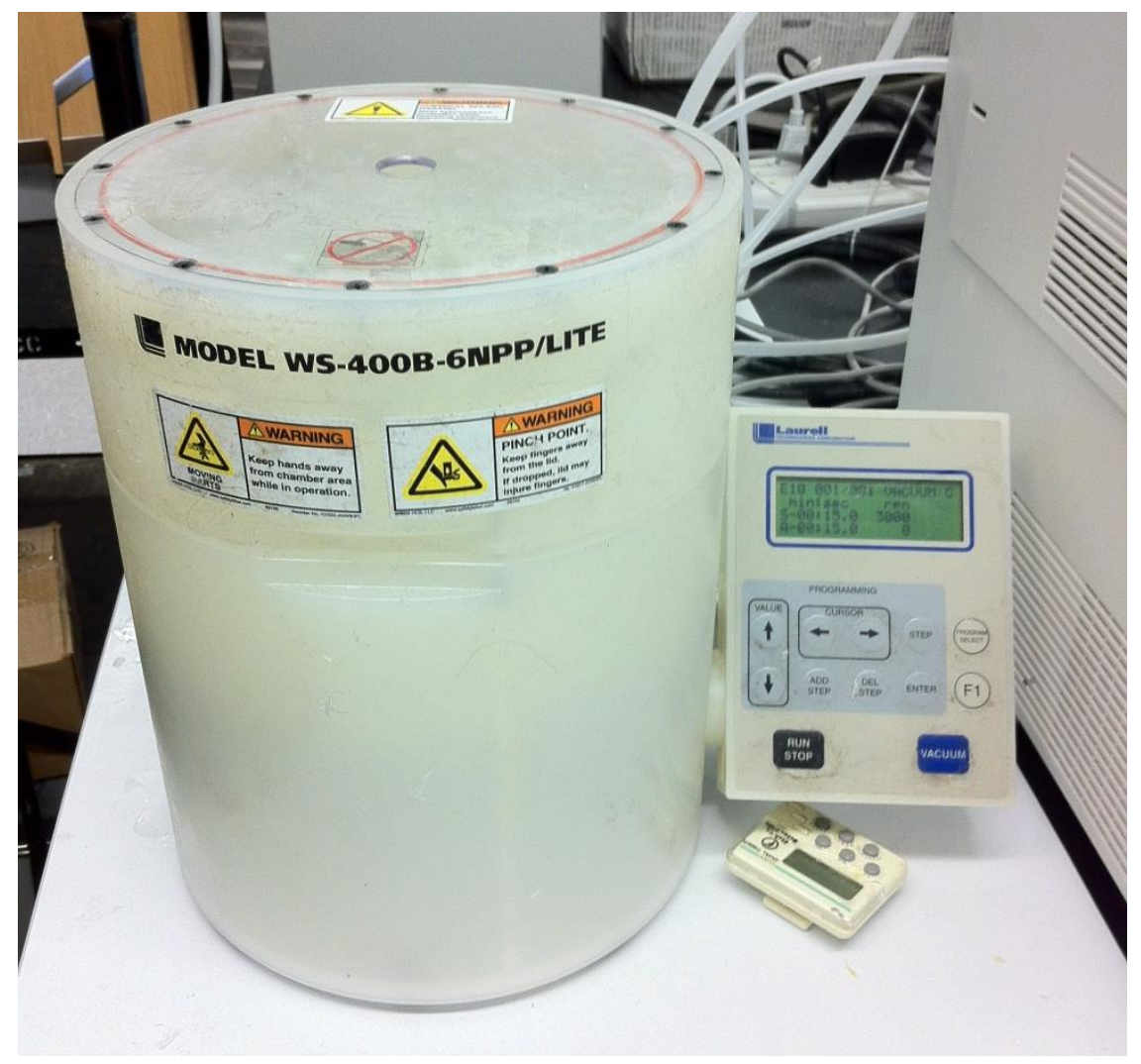

FIGURE 4-Laurell Technologies spin coater used for cover slide coating.

\subsection{Scanning Electron Microscope}

In order to topographically analyze the spin coated samples, a scanning electron microscope (SEM), LEO Supra 35 (Carl Zeiss; Oberkochen, Germany) was employed in order to visualize the surfaces at the resolution needed for analysis. Imaging samples using an SEM involves sending electrons directly to the entire surface of a specimen. The electrons subsequently bounce off of the surface of the specimen and back to a detector which enables the SEM to construct an image of the specimen. 


\subsection{Sputter Coating Instrumentation}

Since the specimen imaged for this study are polymeric in nature and would thus absorb the electrons projected by the SEM, the specimen must be first sputter coated with a thin film of gold to enable SEM imaging. The sputter coating was performed using a PS2 Coating Unit (International Instruments; Santa Clara, CA). Briefly, a small layer of gold is deposited on the samples surface through delivering gold particles to the surface. The gold particles are delivered through release from a gold target by plasma which is created through applying an electric field to inert gas.

\subsection{Data Processing}

UV-VIS data was first obtained by means of the COM port connection from the spectrometer to the computer. All of the .txt files from all of the samples were compiled into one single Excel File (Microsoft Corp., city, state). The degradation rates were computed from the data attained in the Excel spreadsheets. Further data analysis will be described in detail in the proceeding section.

\section{PROCEDURE}


The methods used to conduct this study are described in the following section. First, the initial processing steps of the raw materials prior to mixing are described including their development. Next, the procedures for ultrasonic and mechanical mixing are outlined followed by the procedure to determine the optimal mixing method. Finally, the in-vitro degradation rate quantification and method of FG/PLGA polymer mixtures with differing relative concentrations is described.

\subsection{Material Preparation}

Prior to the combination of the polymers used in this study, both raw materials were subjected to pre-mixing protocols. The material preparation described in this section was performed before all mixing, testing, or use occurred of either polymer. First, PLGA dissolution will be discussed followed by FG heating.

\subsubsection{Poly-lactic-co-glycolic acid dissolution}

Poly-co-lactic-glycolic [75:25] acid (PLGA) procured from Lactel Absorbable Polymers Company (Pelham, AL) in solid form was dissolved in chlorobenzene. Berry et al. identified chlorobenzene as the optimal solvent for the direct-write fiber drawing method with PLGA due to its ability to dissolve PLGA at room temperature [10]. Berry et al. also determined that the ideal concentration of PLGA in chlorobenzene was between $29-30 \%$ (\%w PLGA/total \%w) for the successful fabrication of fibers, with the greatest amount of success occurring when the concentration is on the higher end of the range. Therefore, a 30\% concentration of PLGA to chlorobenzene was used in this study. 
To create PLGA solutions, a glass vial, with screw cap removed, was first placed onto a scale and tared. The desired amount of PLGA was placed in the vial and the weight of chlorobenzene was calculated based upon the measured PLGA weight. The chlorobenzene was then added directly into the vial in drop-wise fashion by use of a glass transfer pipette. Once the desired amount of chlorobenzene was added, the cap was then replaced and wrapped with parafilm to prevent solvent evaporation. Complete PLGA dissolution at room temperature generally took 24-48 hours before use.

\subsubsection{Fish Glue Heating}

Throughout this study, Norland Products Inc. (Cranbury, NJ) High Tack Fish Glue was heated prior to mixing. The heating process was developed to optimize the fiber drawing ability of FG by increasing the viscosity through the removal of excess water present. First, a direct heating method, via a glass beaker with a specified amount of FG was placed on a hot plate. This method was quickly abandoned due to the viscosity of FG and non-uniformity in the heating of the FG was observed, even with stirring. For more uniform heating, a double-boiler method was developed, which involved placing a beaker of FG inside a beaker of water on a hot plate. However, extreme variations in FG/PLGA polymer drawing ability and degradation rates were observed. During initial work, it was observed that the source of variation in the drawing ability and degradation rate of the FG/PLGA polymer could be due to the heating process. During the heating process, the water boils in the outside double-boiler beaker expelling water vapor. It was hypothesized that this vapor could then subsequently 
condense in the beaker of FG, thereby, retarding the efforts of water removal. To remove this potential source of variability, the water bath was replaced with a sand bath.

The sand bath procedure consisted of heating the external $250 \mathrm{~mL}$ beaker filled with sand to $100^{\circ} \mathrm{C}$.. Once the sand bath reached the desired temperature, a $50 \mathrm{~mL}$ beaker filled with $15 \mathrm{~mL}$ of FG and a magnetic stir bar was placed in the sand bath. The sand bath was maintained at a temperature of $100^{\circ} \mathrm{C}$ and the $\mathrm{FG}$ beaker was heated to $100^{\circ} \mathrm{C}$ for 10 minutes with constant stirring. After ten minutes, the beaker of FG was removed from the sand bath and the FG was transferred to a plastic centrifuge tube. Before the tube was capped for storage or use, the FG was allowed to return to room temperature.

\subsection{Fish Glue Solvent Concentration Determination}

Norland High Tack Fish Glue is comprised of Gelatin, Water, and Phenol, as stated on the product's labeling. The exact amount of each component is not specified in the packaging and we were unable to obtain these specifications from Norland Products. To quantify the percentage of solute (gelatin and phenol) versus solvent (water) concentration of stock FG and heated FG the following procedure was used. First, a 15 $\mathrm{mL}$ sample of heated FG was prepared using the sand bath heating method described in the previous section. The following steps were performed for five replicates of both a stock FG sample group and a heated FG sample group. A glass microscope cover slide was weighed and recorded. A $25 \mu \mathrm{L}$ sample of FG was deposited via pipette onto the center of the cover glass. The cover glass with fish glue was immediately returned to the scale and the weight was recorded (Note: this step was performed as quickly as possible 
to measure full weight before evaporation commenced). All samples were placed in a laminar flow hood for 24 hours and allowed to dry at room temperature and humidity. After drying, each glass cover slide was weighed to determine the final dry weight.

\subsection{Gel Permeation Chromatography Measurements}

Samples of Type I collagen from calf skin, Type II collagen from chicken, Type IV collagen from human, cold water fish gelatin, and bovine gelatin were obtained from Sigma-Aldrich Canada. Norland High Take Fish Glue was obtained from Norland Products Inc, Cranbury, NJ. Gel Permeation Chromatography (GPC) was performed to determine the molecular weight of FG through regression analysis of by using collagen and gelatin samples with known molecular weights using a Waters Ultrahydrogel 250 column with a mobile phase of $20 \%$ acetonitrile in $0.1 \mathrm{M}$ sodium nitrate with a 0.3 $\mathrm{ml} / \mathrm{min}$ flow rate using a Waters Binary Pump. Column temperature and pressure were maintained at $30^{\circ} \mathrm{C}$ and $285 \mathrm{~mm} \mathrm{Hg}$, respectively. Each sample was dissolved in the mobile phase at a concentration of $0.7 \mathrm{mg} / \mathrm{mL}$. Samples were detected using a Waters Refractive Index RI detector and a Waters UV/Vis detector at a detection wavelength of $280 \mathrm{~nm}$ for a 60 minute elution time.

\subsection{Mixing Method Determination}

The ability to mix PLGA with FG in a reliable and repeatable manner is necessary for its potential use as a biomaterial. In this section, the procedures for each of the two mixing methods tested are first described. Then, the test method to determine level of mixing is described. 


\subsubsection{Ultrasonic Mixing}

In preliminary investigations, the initial mixing method explored consisted of placing PLGA and FG into a glass vial at their desired relative concentration (\%w FG/total \%w). The vial was then capped, wrapped with parafilm, and placed in the ultrasonic cleaner. After placement, the ultrasonic cleaner was activated for a full cycle time of 60 minutes. Each vial mixed was be subjected to four cycles, total mixing time of 4 hours. This 4 hour mixing time was qualitatively determined to be ideal prior to this thesis. After each cycle, the water in the cleaner was replaced to ensure excess water heating did not occur. Following the final cycle, the glass vial was removed from the ultrasonic cleaner.

\subsubsection{Mechanical Mixing}

A second mixing method was developed during the course of this thesis. Briefly, FG and PLGA were combined in a glass vial at their desired relative concentrations. The glass vial was then positioned under the Heidolph overhead stirrer to the point that the bottom tip of the steel mixing wire was approximately $1 \mathrm{~mm}$ from the bottom of the vial. After placement, the mixer was turned on, placed at a rotation setting of $100 \mathrm{rpm}$ and mixed for different amounts of time (30 minutes, 1 hour, 2 hours and 4 hours). Once the desired amount of time was reached, the vial was removed from the mechanical mixer, capped, and was ready for use.

\subsubsection{Mixing Method Testing}


When mixing is initiated, the polymers are in a distinct suspension due to the insolubility of gelatin, the polymer component of FG, with chlorobenzene. However after mixing is induced, chlorobenzene soluble PLGA begins to mix into the water phase, containing FG. Visually this occurs with a color change from caramel to off-white of the water soluble phase containing FG. Due to this optical change, the level of mixing was determined through UV-VIS absorbance spectra measurements. For testing, three samples of each of the following solutions were created: FG, PLGA, 4 hour ultrasonic mixing, 4 hour mechanical mixing, 2 hour mechanical mixing, 1 hour mechanical mixing, and 30 minute mechanical mixing. FG and PLGA samples were created as standards; each sample was created as described in the Material Preparation section. The absorbance spectra for all samples were measured with a distilled water blank. To prevent signal saturation, all samples were diluted serially from their vial to a $1 \%$ concentration in distilled water in the specimen cuvette. Absorbance measurements were taken at wavelengths varying from $190-350 \mathrm{~nm}$ at $1 \mathrm{~nm}$ increments. Analysis was performed at wavelengths of 260 and 280 due to their importance with DNA and protein spectrophotometry.

\subsection{Degradation Characterization}

This section outlines the determination of the degradation rate for differing FG/PLGA solutions with respect to their relative weight concentrations. A spin coating method of sample fabrication was chosen over the direct-write method due to its ease of use, speed of fabrication, and ability to produce samples of similar properties to directwrite fibers. First, the surface area to volume calculation is explained. Then, the test 
procedure is outlined. Finally, the method for image analysis of a representative group is stated.

\subsubsection{Surface Area to Volume Calculation}

Due to the fact that hydrolysis, the degradation mechanism for PLGA, is increased with an increase in the surface area to volume ratio $(\mathrm{SA} / \mathrm{V})$ of the scaffold, the spincoated samples surface area to volume ratios must maintain consistency with direct-write fibers. In order to maintain a surface area to volume ratio consistent with the application of fibers, the following equations and calculations were used. A characteristic PLGA direct-write fiber was modeled as a perfect solid cylinder. To calculate the SA/V ratio of the fiber, the top and bottom faces of the cylinder were neglected because they are connected to a supportive frame. Equation 1 shows the Surface Area calculation and Equation 2 depicts the Volume calculation of a fiber. Dividing Equation 1 by Equation 2 yields the SA/V ratio shown in Equation 3. For the spin coating application:

$$
\begin{aligned}
& \text { Surface Area }=2 \pi r h \\
& \text { Volume }=\pi r^{2} h
\end{aligned}
$$




$$
\frac{\mathrm{SA}}{\mathrm{V}}=\frac{2}{\mathrm{r}}
$$

The substrate used for spin coating was a circular glass covers slide. Equation 4 shows the simple surface is calculation of the circle. To calculate the SA/V ratio for the cover slide shown as Equation 5, Equation 4 was divided by an unknown Volume (Vu).

$$
\begin{gathered}
\text { Surface Area }=\pi R^{2} \\
\frac{S A}{V}=\frac{\pi R^{2}}{V u}
\end{gathered}
$$

Equilibrating the Equation 3 with Equation 5 and solving for $\mathrm{Vu}$ yields Equation 6.

$$
V u=\frac{\pi R^{2} r}{2}
$$

Assuming that the presence of FG in PLGA will not drastically change fiber diameter, the radius of the representative fiber, $r=8.435$, was gleaned from a diameter measurement of a 75:25 PLGA fiber at 30\% in chlorobenzene by Berry et al. of 16.87 $\mu \mathrm{m}$. The glass cover slides used as a substrate for spin coating had a radius, $\mathrm{R}$, of 12.5 $\mathrm{mm}$. Solving for Equation 6 with the previously stated values of $\mathrm{R}$ and $\mathrm{r}$ and converting from meters to liters, yields the unknown $\mathrm{Vu}$ of $2.1 \mu \mathrm{L}$. The actual injected volume added for spin coating was $20 \mu \mathrm{L}$ which was the calculated Vu plus a safety factor to 
account for polymer loss during the spin process as well as volume loss during the drying process.

\subsubsection{Degradation Rate Measurements}

Degradation measurements were taken from samples of mixed FG/PLGA polymers at percent relative concentrations of $65 / 35,70 / 30,75 / 25$, and $80 / 20(\% \mathrm{w}$ FG/\%w PLGA). Samples were prepared using the methods discussed above. Briefly, for each sample, PLGA was dissolved in chlorobenzene at a $30 \%$ concentration in a glass vial. Then, an excess of heated FG was heated to be used for all mixtures. Finally, FG was added to the PLGA at one of the desired concentrations mentioned previously and mixed mechanically at $100 \mathrm{rpm}$ for 1 hour. The amount of time used for mechanical mixing was determined through data analysis of the mixing test previously described.

After mixing, 24 spin-coated specimens were created from each of the four different mixtures. Six time points of 12 hours, 1 day, 2 days, 6 days, 10 days, and 14 days were used and for each time point three replicates were fabricated. To create a specimen, a clean glass cover slide was secured on the spin coater. Then, a $25 \mu \mathrm{L}$ volume of the desired polymer mixture was deposited in the center of the cover slide. The sample was then spun within the spin coater for 15 seconds at a speed of $3000 \mathrm{rpm}$. After spinning, the cover slide was removed from the spin coater and allowed to dry for 24 hours.

Day 0 of the study began with each dry specimen being weighed to obtain the initial weight, $\mathrm{W}_{\mathrm{o}}$. Replicates of the same time point and FG/PLGA concentration were 
placed in a petri dish and $1.5 \mathrm{~mL}$ of $1 \mathrm{X}$ Phosphate Buffered Saline (PBS), was added. Then, all specimens were placed in an incubator at $37^{\circ} \mathrm{C}$. Every 2 days, old PBS was removed and replaced with fresh PBS. At each time point, specimens labeled with the corresponding time point were removed from the incubator, aspirated with PBS, and allowed to dry for 24 hours. Once a specimen was dried, the final weight was measured, $\mathrm{W}_{\mathrm{i}}$.

\subsubsection{Degradation Topographical Analysis}

Scanning Electron Microscopy imaging was used to qualitatively determine topographical changes in the surface of the FG/PLGA polymer mixture as the material degraded. A single replicate from each time point (including $\mathrm{t}=0$ ) of the $65 / 35 \mathrm{FG} / \mathrm{PLGA}$ mixture group was reserved after the Degradation Rate Analysis procedure described in the previous section for SEM analysis. Each cover slide was placed on the SEM sample plate using doubled sided carbon conductive tape. Extreme care was taken during this process to mount each specimen flatly as well as to maintain time point numerical order for specimen labeling during SEM imaging. The sample plate was then sputter coated with a thin layer of conductive gold using the PS2 Coating Unit. To sputter coat, the sample plate was placed inside the sputter chamber and the vacuum pump was activated to establish a pressure of 0.1 torr. A 1 kilovolt electric field was used to create plasma which bombards a gold target freeing gold atoms which then attach in layer form to the sample. The sputter coating process was carried out for 90 seconds. After sputter coating, the sample plate was placed in the SEM sample carousel. The SEM chamber 
was closed, sealed, and a vacuum was established. An electron beam voltage of $5 \mathrm{kV}$ was established and the secondary electron detector was turned on. Images were captured of each time point specimen at similar zoom settings, with scale bars of approximately $2 \mu \mathrm{m}, 20 \mu \mathrm{m}$, and $100 \mu \mathrm{m}$. Clear images were obtained through manipulation of wobble, stigmation, brightness, and contrast settings.

\section{RESULTS AND DISCUSSION}




\subsection{Fish Glue Concentration Results}

The percentage of water versus solid components in FG was determined for stock FG and heat treated fish glue. Five specimen of each heat treatment weighing approximately 40 mg were allowed to sit over night to dry. Full results of the recorded measurements can be found in Appendix 1. The final weights proved that the heating process was indeed evacuating liquid from the stock fish glue yielding a more concentrated final product. This is shown by a Mann Whitney Median test between the two samples. A two sample student's t-test would have been preferred; however, as FIGURE 5 shows below, the Noheat sample's FG concentration percentage was not normally distributed ( $\mathrm{p}-$ value $=$ 0.007 in test for normality). However, the heated group of FG was shown to have a larger span in percent solid concentration which could be due to variability in the heating process. Figure 5 also shows that the stock fish glue has a solid concentration of $56.87 \%$ while the heated samples have a concentration of 52.70\%. FIGURE 6 illustrates the details of the Mann-Whitney test, which suggests that the Heated FG group's concentration is 4 percent higher this implies that the heating process causes a sample of stock fish glue to lose approximately 4 percent of its mass. 


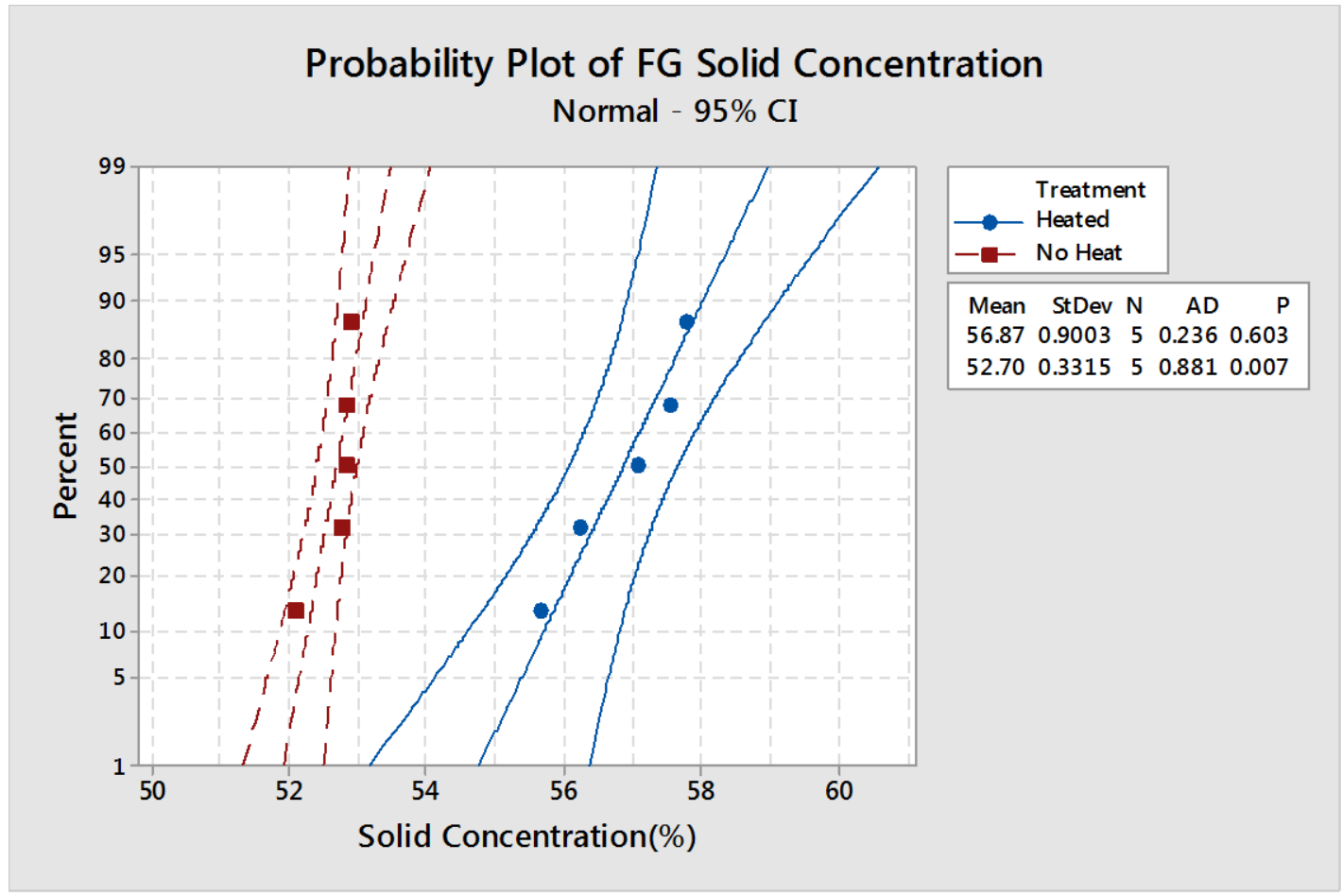

FIGURE 5-Probability plot of Heated and No Heat \% Solid Conc.

Mann-Whitney Test and CI: Heated FG Solid Conc., Not Heated FG Solid Conc.

N Median

Heated FG Solid Conc. $\quad 5 \quad 57.070$

Not Heated FG Solid Conc. $5 \quad 52.850$

Point estimate for $\mathrm{\eta} 1-\mathrm{\eta} 2$ is 4.220

96.3 Percent CI for $\eta 1-\eta 2$ is $(2.810,5.020)$

$\mathrm{W}=40.0$

Test of $\eta 1=\eta 2$ vs $\eta 1 \neq \eta 2$ is significant at 0.0122

The test is significant at 0.0119 (adjusted for ties)

FIGURE 6-Mann-Whitney Test of Heated vs No Heat \% Solid Conc. 


\subsection{Gel Permeation Chromatography Results}

Refractive Index (RI), a measurement made from visible light, and $280 \mathrm{~nm}$ spectra were successfully measured for each sample of FG and the standard animal gelatins and collagens. FIGURE 7 and FIGURE 8 illustrate the RI and $280 \mathrm{~nm}$ spectra of FG, respectively. FIGURE 9 and FIGURE 10 illustrate the RI and $280 \mathrm{~nm}$ spectra of FG and all animal gelatins and collagens, respectively. All standards molecular weight and elution time until first peak for RI and 280 are shown in TABLE 1. Standard curves of molecular weight versus elution were generated for each spectra (RI and 280) along with a linear fit shown in FIGURE 11 and FIGURE 12. Higher molecular weight samples were seen to have a shorter elution time for a peak with the RI sensor as compared to lower molecular weight samples; whereas, the elution time for a higher molecular weight sample was longer than a lower molecular weight sample with the $280 \mathrm{~nm}$ sensor. This discrepancy could be due to a couple of factors: the sensors are in series of each other, potentially causing a time lag and a constituent of a sample could cause a signal with one sensor and not the other, i.e. the $280 \mathrm{~nm}$ sample could cause excitation of a constituent producing a signal that would not be seen with the RI sensor. Molecular weight values for the standards were gleaned from the Extracellular Matrix Factsbook (Ayad et al. 1998). The linear fit from each detection method was used to determine the molecular weight of FG based on elution time. The curve for the RI detector and $280 \mathrm{~nm}$ detector yielded a molecular weight prediction for FG of 176,500 Da and 165,200 Da, respectively. The variable prediction of molecular weight could be due to technical difficulties with the Waters GPC pump, a gradient chromatography was not able to be 
run. Having the ability to run a gradient chromatography would allow the elution times of the molecular weights to gain more resolution, thus increasing the accuracy of the standard curve. 


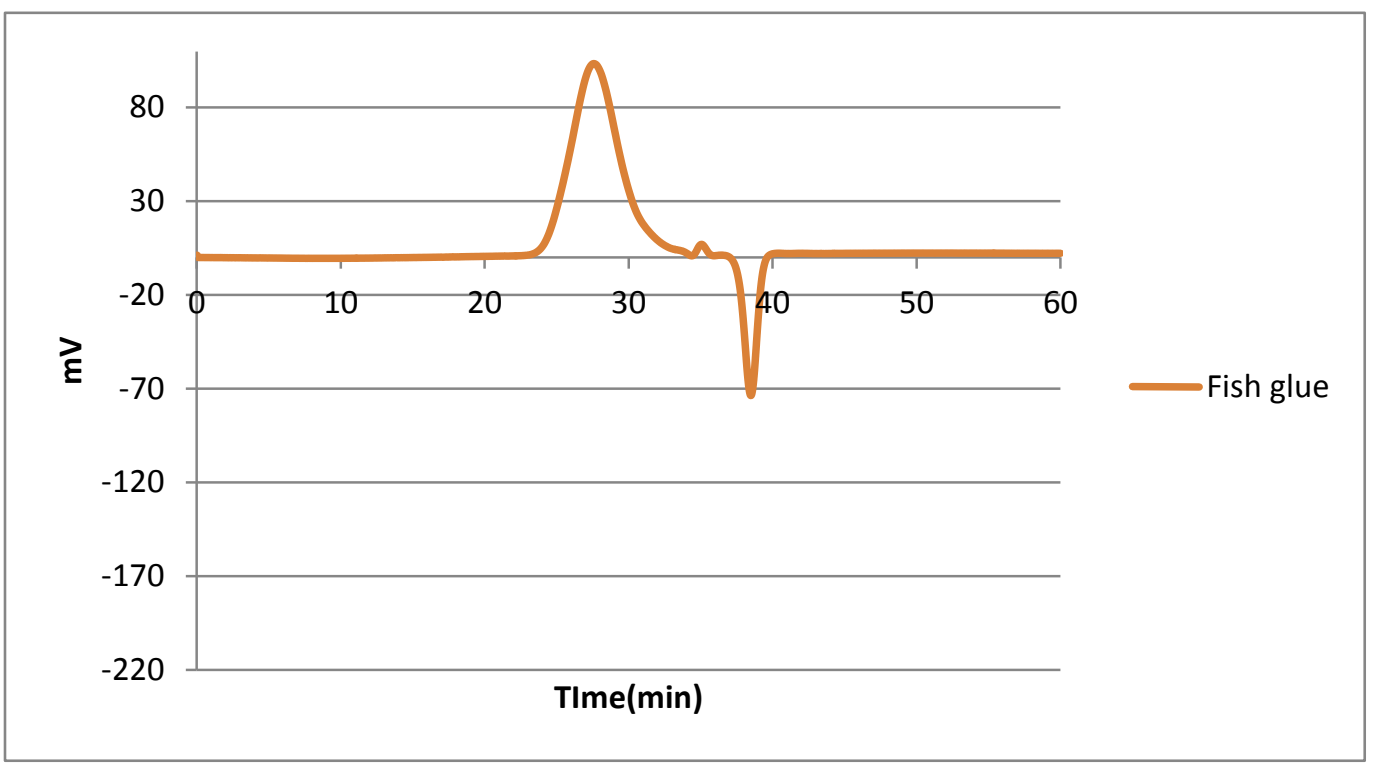

FIGURE 7-Fish glue refractive index spectra.

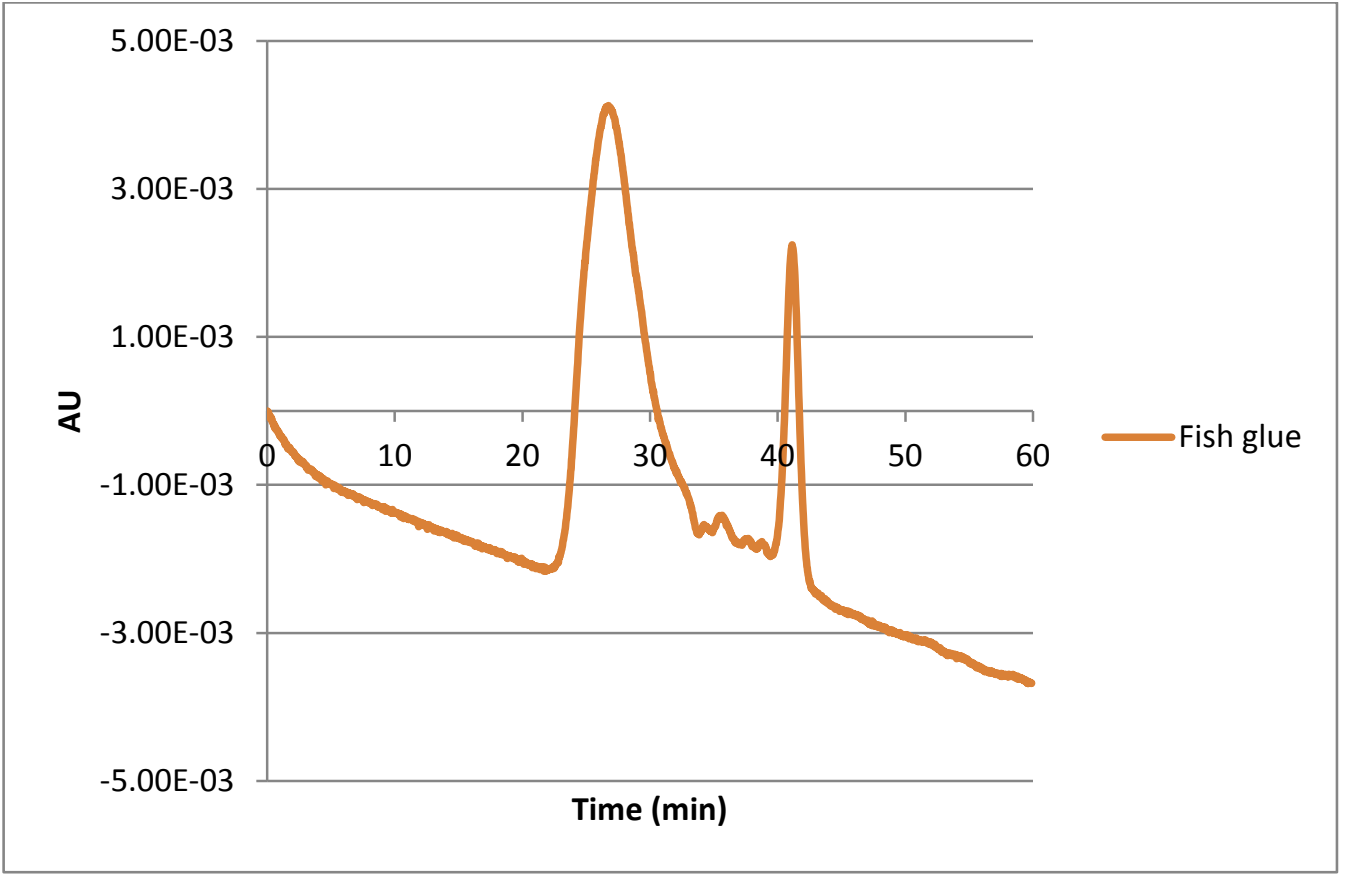


FIGURE 8-Fish glue $280 \mathrm{~nm}$ spectra.

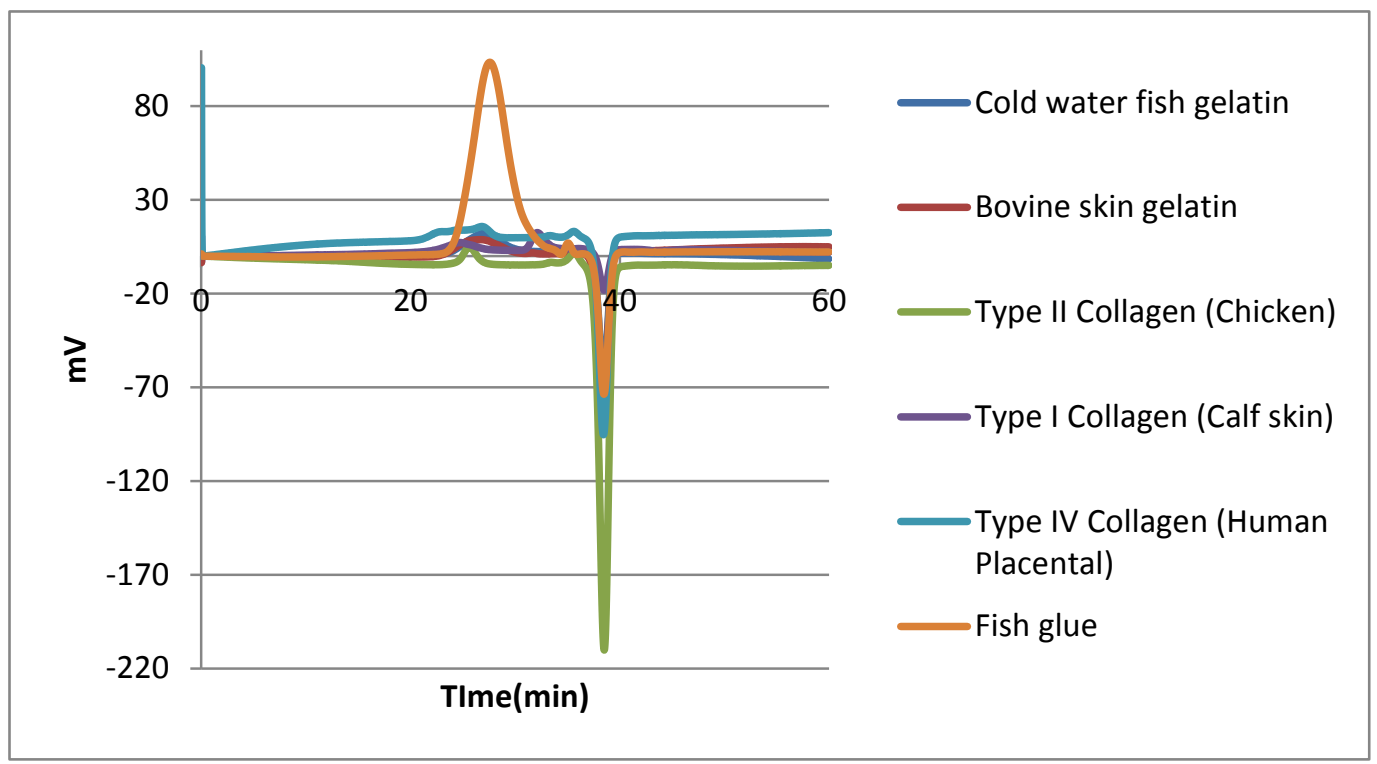

FIGURE 9-All sample RI spectra. 


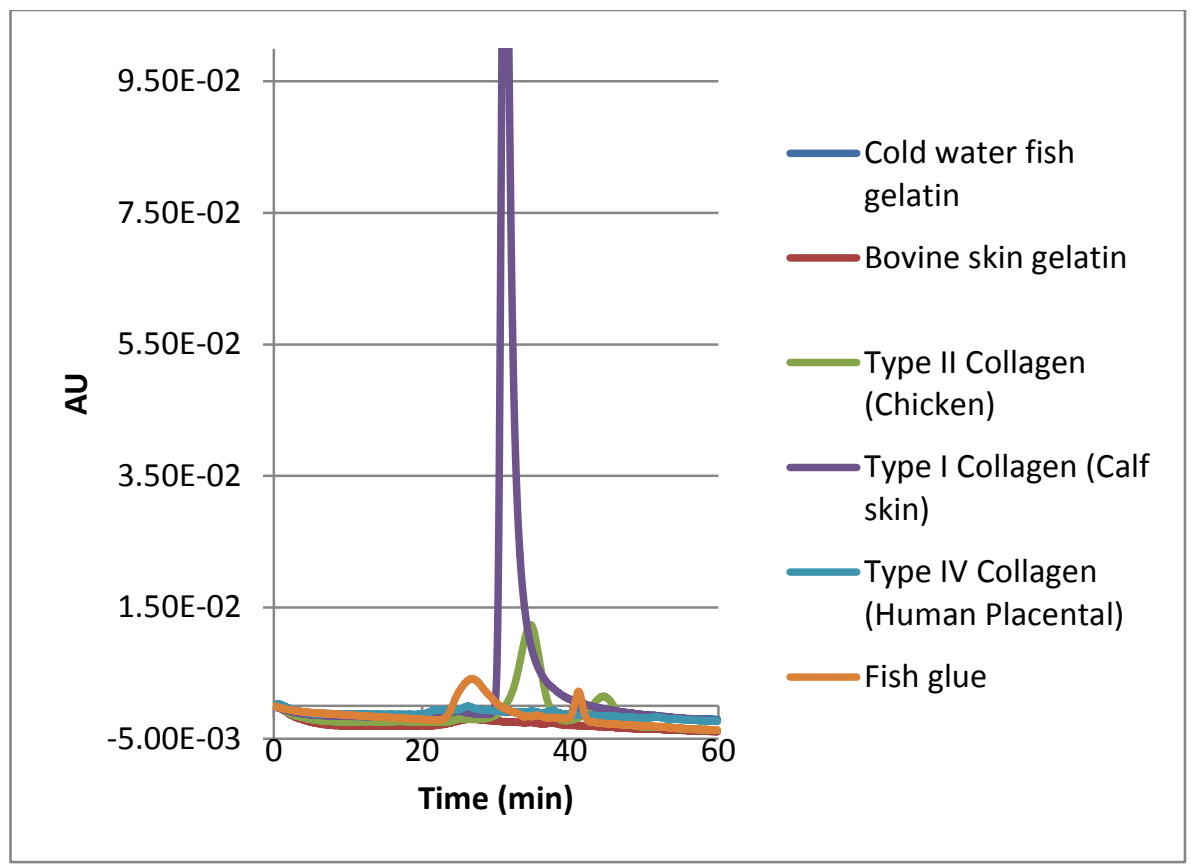

FIGURE 10_All sample $280 \mathrm{~nm}$ spectra.

TABLE $1-$ Standard Elution times and Molecular weights.

\begin{tabular}{|c|c|c|c|}
\hline Standard & $\begin{array}{c}\text { Elution Time } \\
(\mathbf{m i n}) \mathbf{R I}\end{array}$ & $\begin{array}{c}\text { Elution Time } \\
(\mathbf{m i n}) \mathbf{2 8 0}\end{array}$ & $\begin{array}{c}\text { Molecular } \\
\text { Weight(Da) }\end{array}$ \\
\hline Cold water fish gelatin & 27.2 & 26.8 & 169500 \\
\hline Bovine skin gelatin & 26.9 & 26.5 & 171000 \\
\hline $\begin{array}{c}\text { Type II Collagen } \\
\text { (Chicken) }\end{array}$ & 25.6 & 34.7 & 141800 \\
\hline $\begin{array}{c}\text { Type I Collagen (Calf } \\
\text { skin) }\end{array}$ & 24.7 & 31.2 & 138700 \\
\hline $\begin{array}{c}\text { Type IV Collagen } \\
\text { (Human Placental) }\end{array}$ & 26.8 & 26.2 & 161700 \\
\hline
\end{tabular}




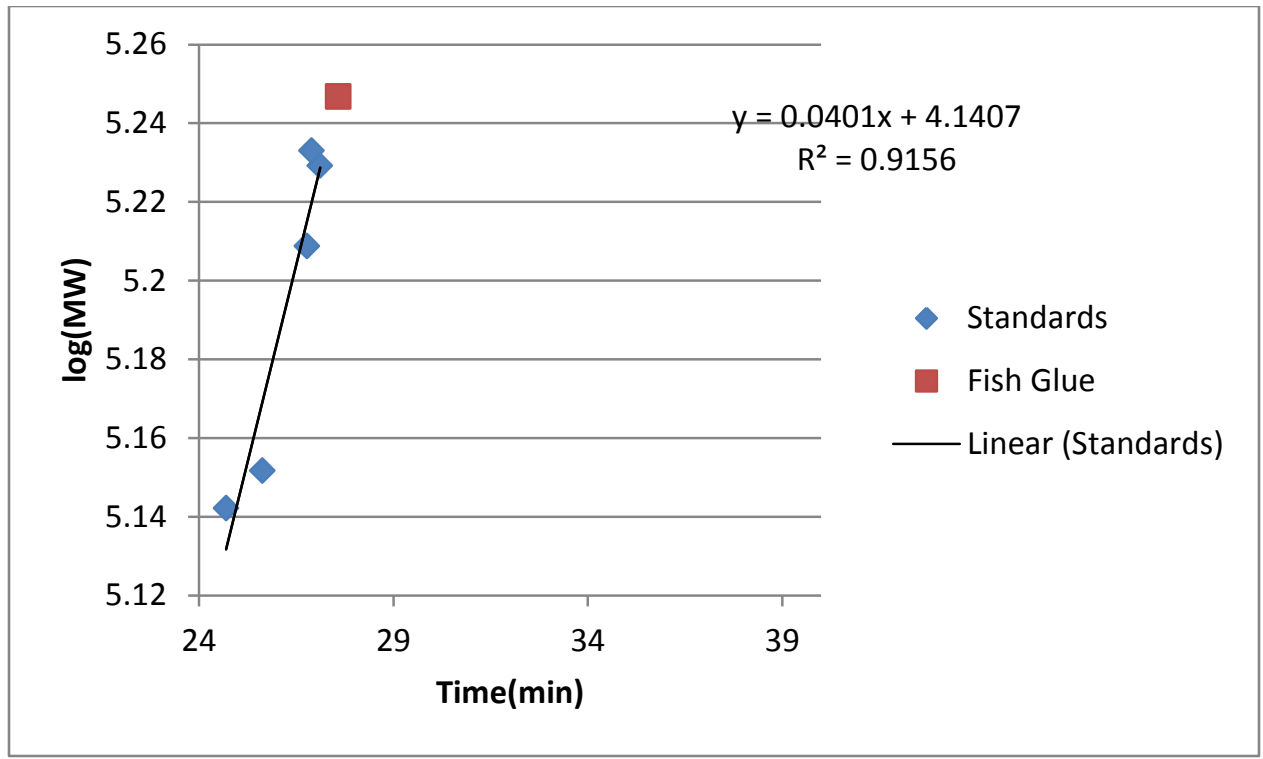

FIGURE $11-$ Standard Curve of RI elution time and MW.

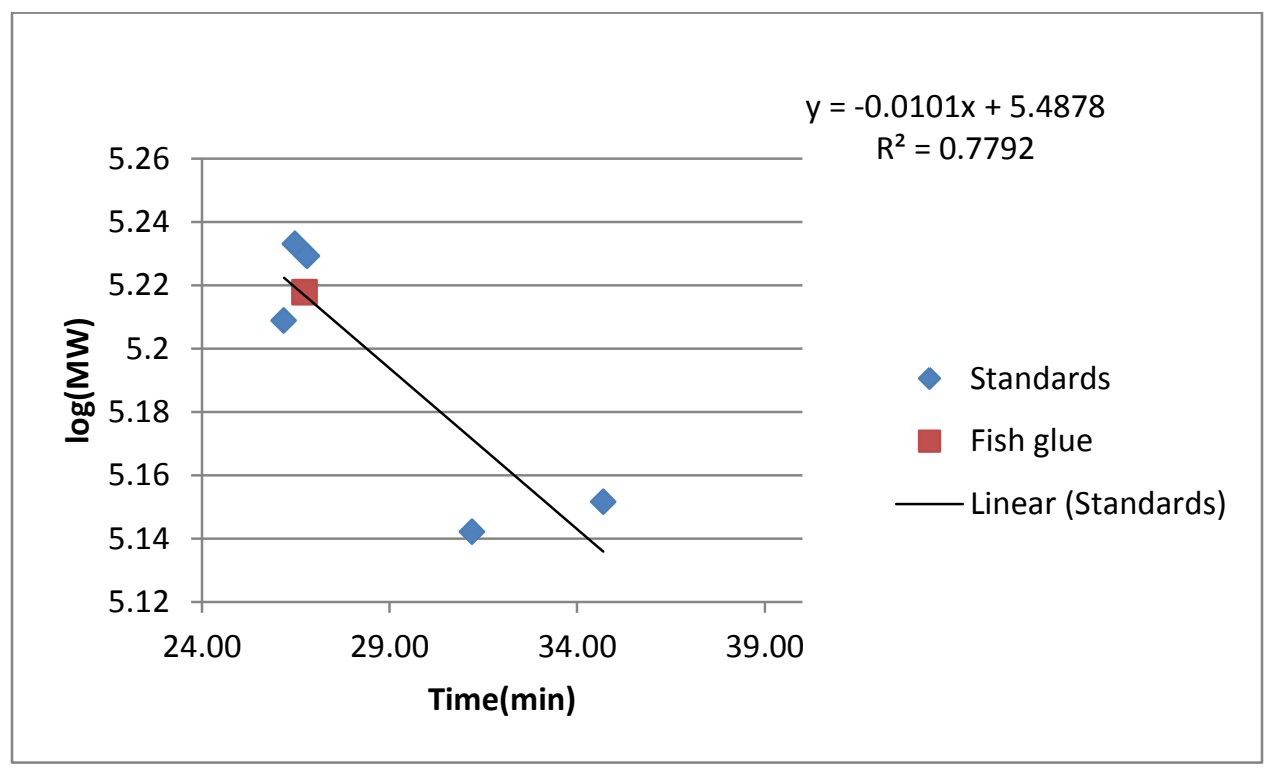

FIGURE 12-Standard Curve of $280 \mathrm{~nm}$ elution time and MW. 


\subsection{Mixing Method Results}

Absorbance spectra were successfully obtained for all samples of different mixing methods and time. The full results of UV-Vis spectra and graphs can be found in Appendix 3. Spectral analysis showed that as the amount of mechanical mixing time increased, the amount of light absorbance for a mixture also increased, potentially indicating that as more PLGA mixes into the FG, it increases its ability to absorb light, as was shown at $260 \mathrm{~nm}$ and $280 \mathrm{~nm}$. Full results can be found in Appendix 2. FIGURE 13 and FIGURE 14 provide an illustration of the average spectra of FG and PLGA at varying mechanical mixing times and a 4 hour sonication. The mechanical mix times of 30, 1 hour, 2 hours, and 4 hours provides a time sequence of mixing levels. The two sample student's t-test between mechanical and sonication at $260 \mathrm{~nm}$ proved significant $(\mathrm{p}=0.001)$ meaning that the absorbance measurements for mechanical mixing were greater than that of sonication as shown in FIGURE 15. However, FIGURE 16 and FIGURE 17 show that the two sample t-tests between sonication and mechanical mixed groups at $280 \mathrm{~nm}$ as well as the ratio $260: 280$, which approximately gives nucleic acid to protein ratio, both prove no statistical differences between mixing groups. Statistically nothing can be proven as to the amount of time necessary to properly mix FG and PLGA mechanically, no tests showed a statistical difference between any mixing times in terms of absorbance at 280 and $260 \mathrm{~nm}$. However, a difference in mixture variation can be shown graphically as seen in FIGURE 18 and FIGURE 19, which show the mean absorbance at $280 \mathrm{~nm}$ and $260 \mathrm{~nm}$, respectively; furthermore, it can be seen that a 1 hour mixing time could potentially be optimal because it is a relatively short amount of time 
among the choices and provides a lower amount of variation against the other groups. Limitations of this testing include: variability of serial dilution causing variability in measured absorbance, not performing a blank of chlorobenzene, and only having three replicates per sample. 


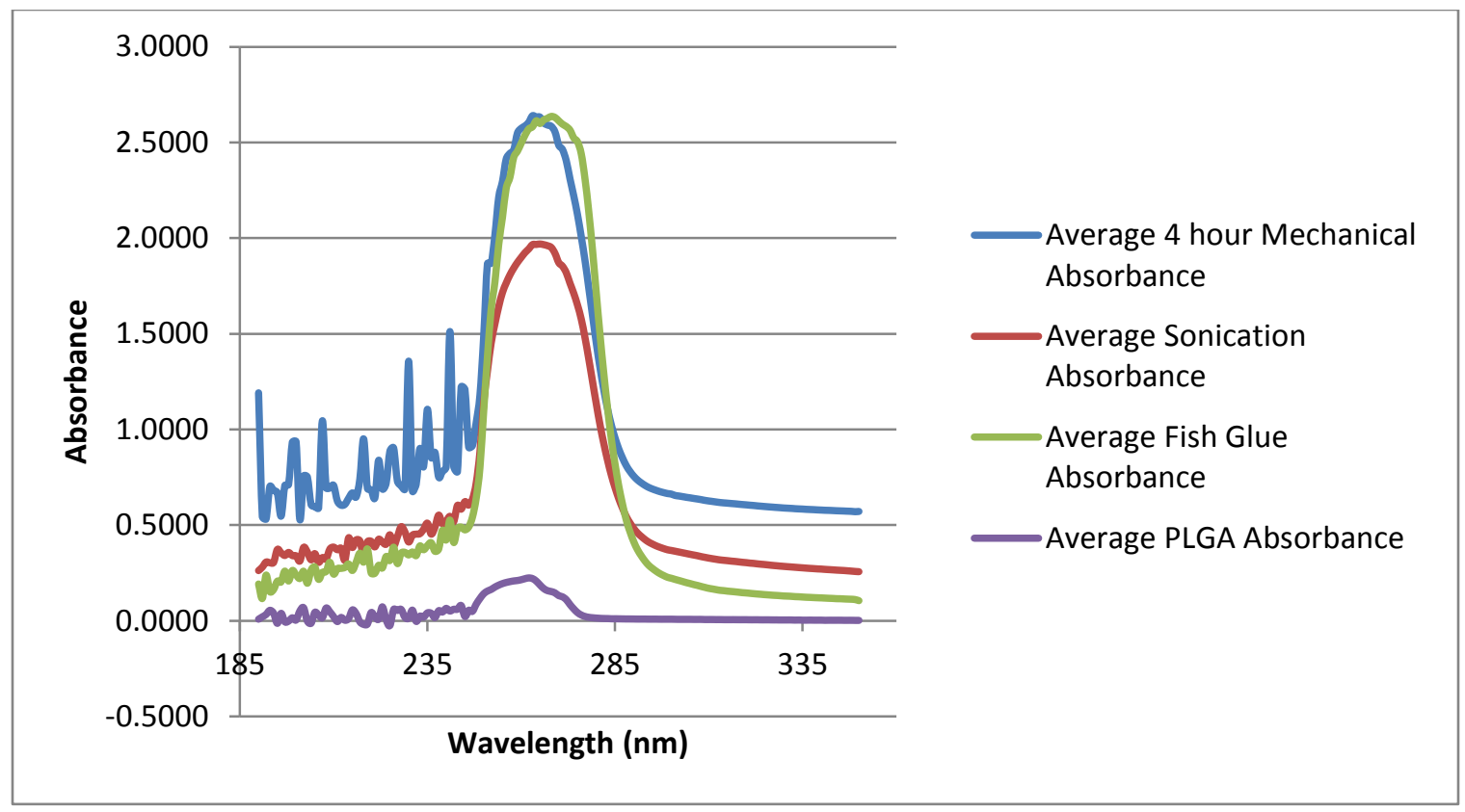

FIGURE 13-Absorbance spectra of FG, PLGA, 4 hr Sonication, 4 hr Mechanical mix.

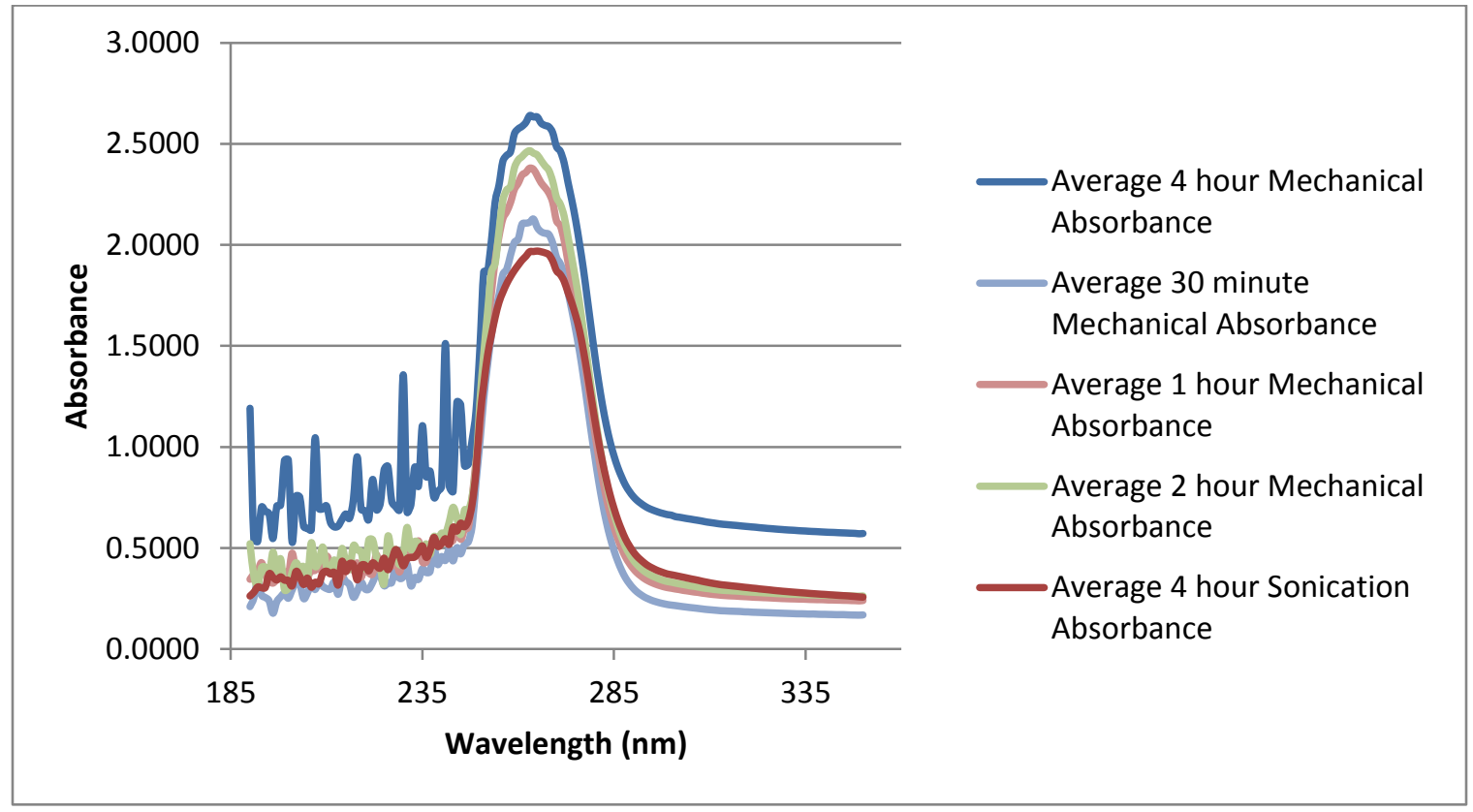

FIGURE 14-Absorbance spectra of timed mechanical mixes and $4 \mathrm{hr}$ Sonication. 


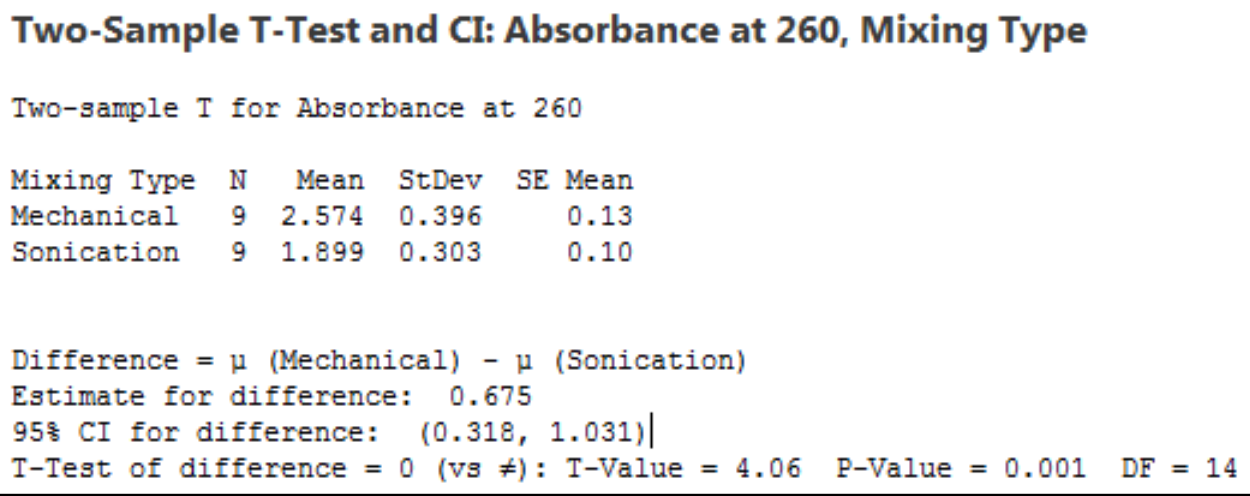

FIGURE 15-Two Sample T-test of Absorbance at 260 of mixing types

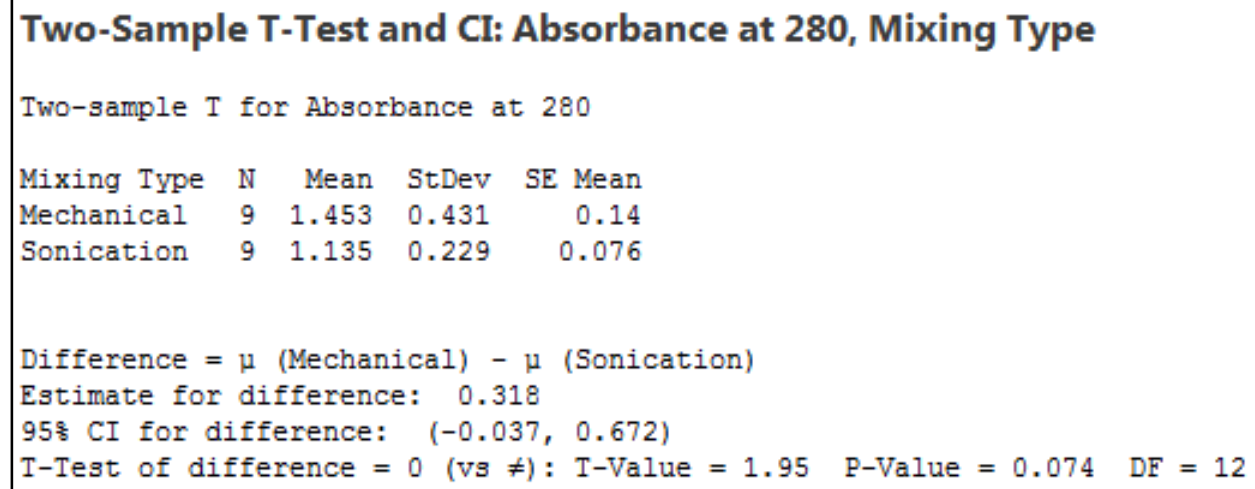

FIGURE 16-- Two Sample T-test of Absorbance at 280 of mixing types 


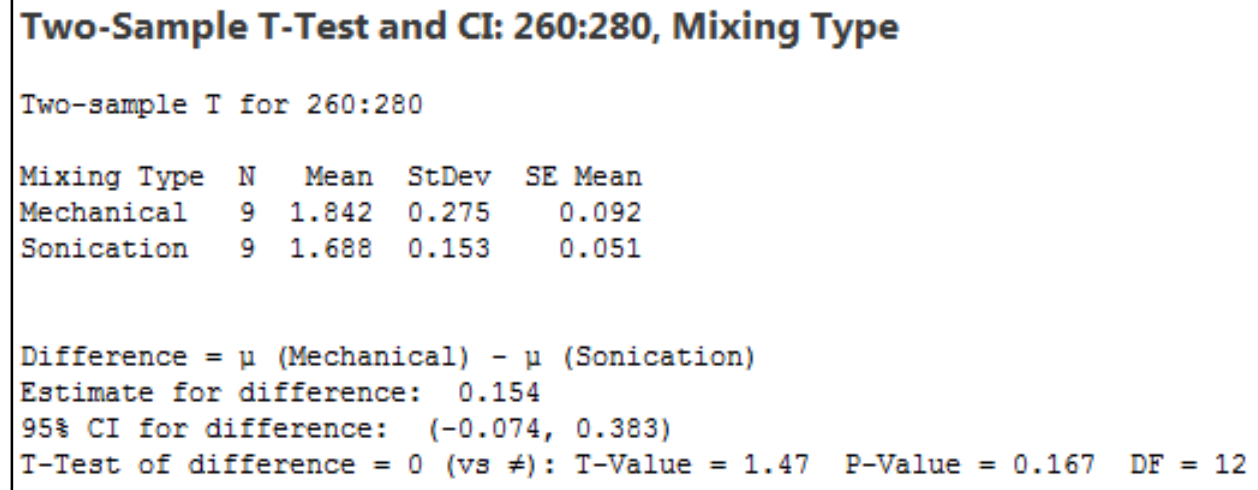

FIGURE 17- Two Sample T-test of Absorbance at 260:280 of mixing types

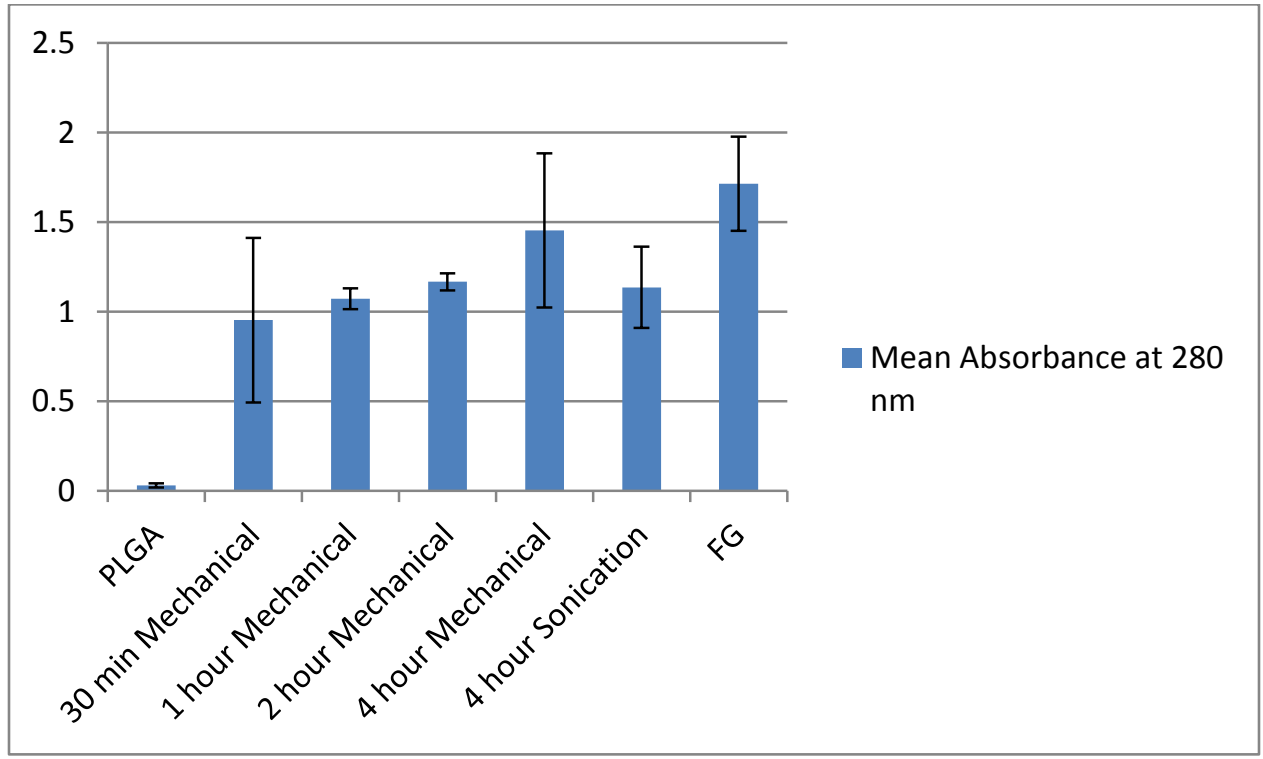

FIGURE 18-Mean absorbance measurements at $280 \mathrm{~nm}$ with standard deviation bars 


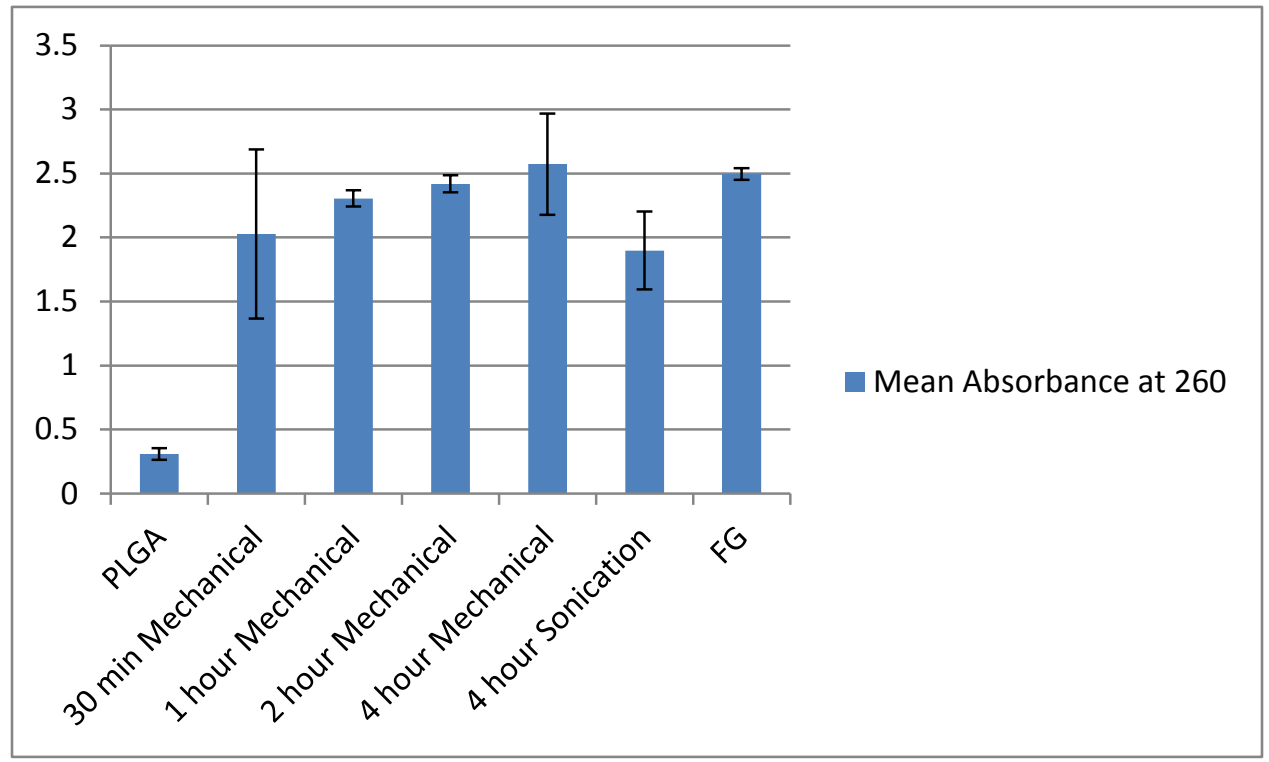

FIGURE 19-Mean absorbance measurements at $260 \mathrm{~nm}$ with standard deviation bars

\subsection{Degradation Study Results and Analysis}

\subsubsection{Degradation Rate Results}

Three replicates of each varying concentration were successfully weighed at 12 hours, 1 day, 2 days, 6 days, 10 days, and 14 days as well as at day zero. The percent weight remaining was calculated for each measured specimen based off of its initial weight. Full data can be found in Appendix 3. The measured results show that all samples lose at least $70 \%$ of their weight after twelve hours and all samples losing at least 90 percent of their weight by day 2. All samples showed a leveling off around 5\% weight remaining at day 14. It is hypothesized that the initial loss of mass is mainly comprised of FG as FG is more readily soluble in water than PLGA; whereas, PLGA dissolves via hydrolysis. No 
findings statistically could be reported to show differences in degradation amounts between the varying mixture concentrations; however, FIGURE 20 provides an illustration of the degradation of the different concentration mixtures of FG/PLGA. Limitations that could have influenced the results obtained include: only having the replicates per time point per concentration combination, not weighing the aspirate liquid to further measure percent loss per time point per specimen, and not measuring standard PLGA and FG only specimen degradation rates.

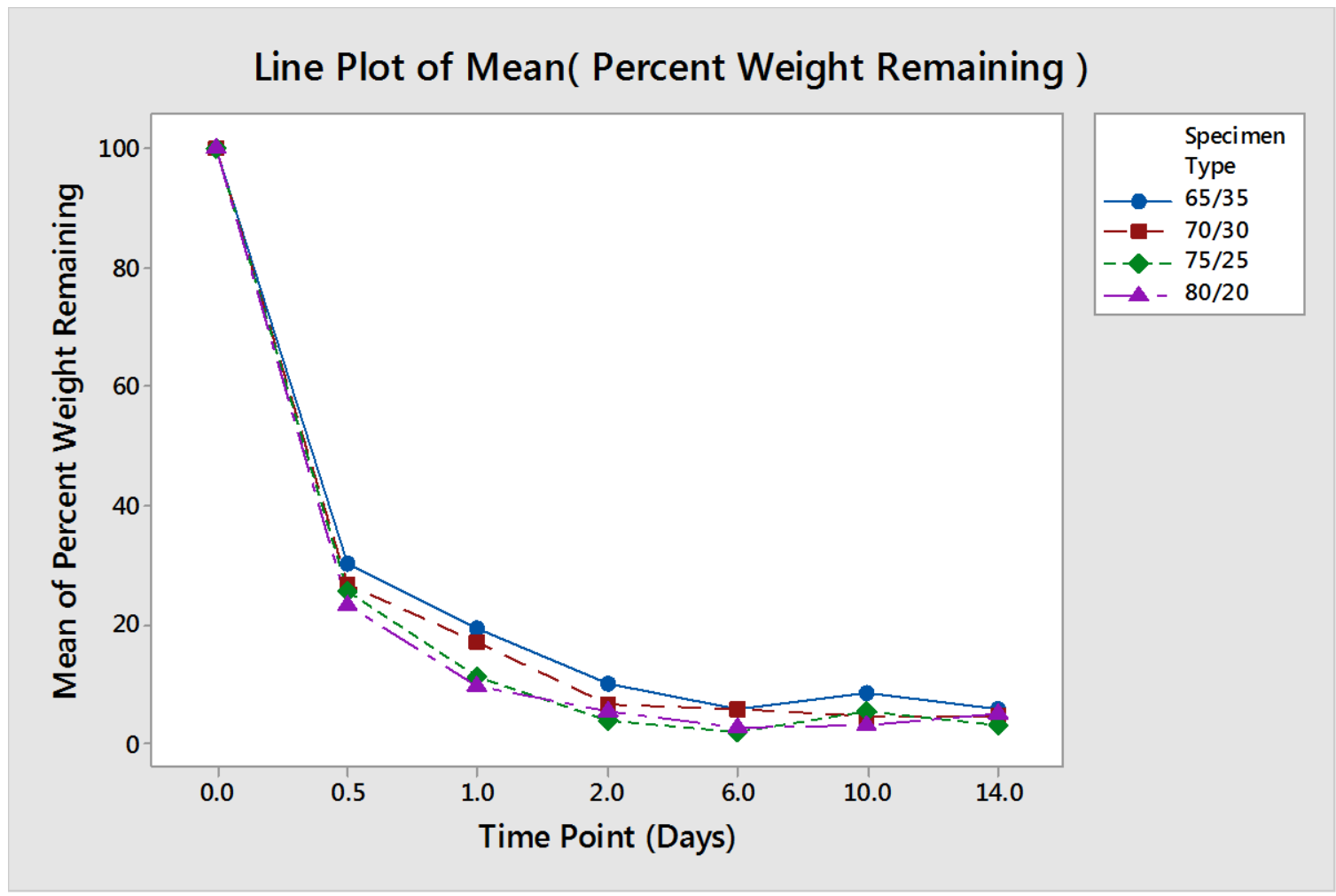

FIGURE 20—Percent Weight remaining by days of different concentrated mixtures. 


\subsubsection{Topographical Analysis}

Due to the large number of specimen and the amount of time necessary to properly SEM image each specimen, the $65 / 35$ concentration was chosen as the representative group to be imaged for this topographical analysis. Full SEM images from each time point can be found in Appendix 4. FIGURE 21 and FIGURE 22 show selected SEM images at time points 5 and 6, respectively. From these images and comparison with the earlier time points, it is potentially shown that a colloid-like mixture has likely been made of FG and PLGA where the PLGA appears as circular artifacts in the later time points as seen in time points 5 and 6.

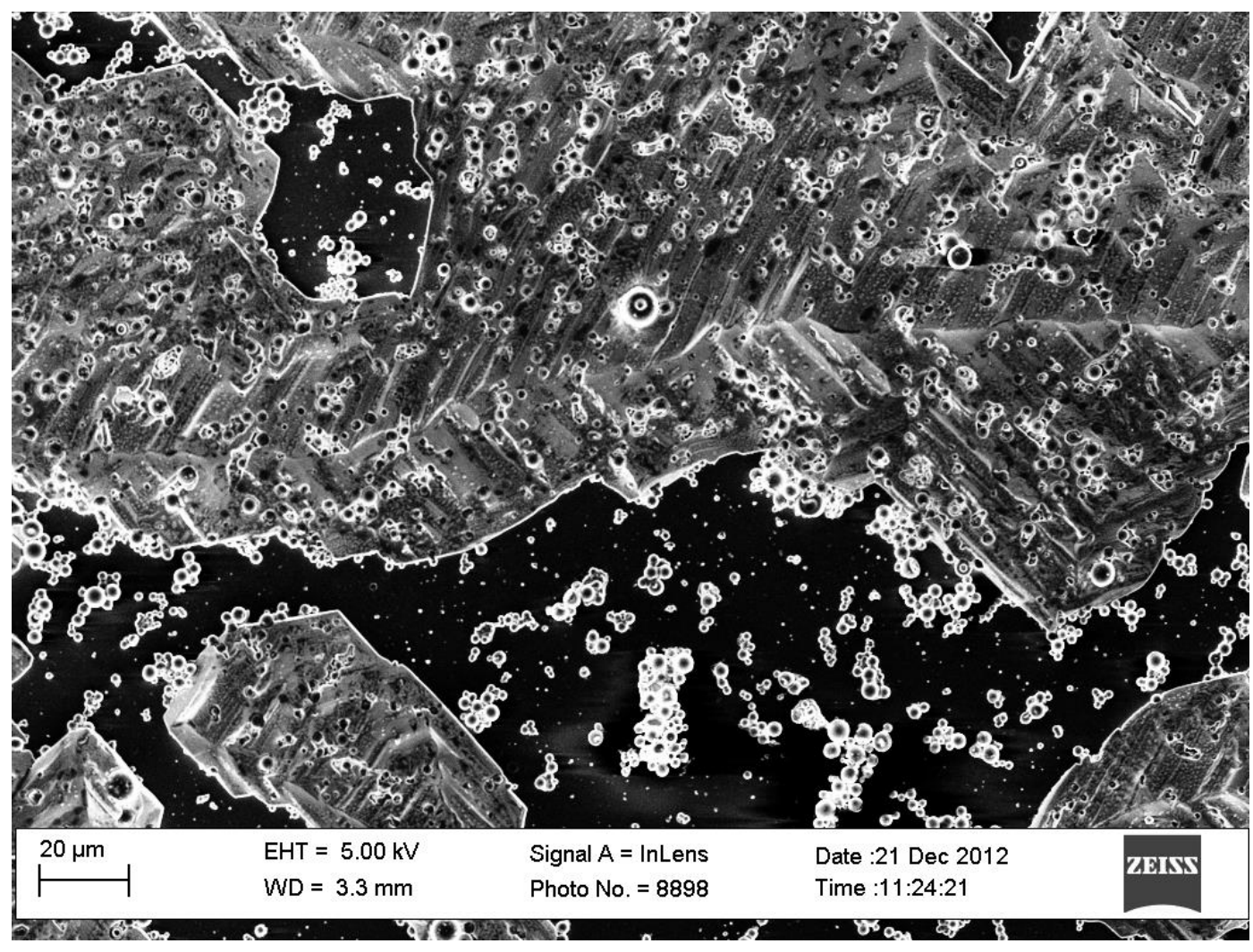

FIGURE 21-SEM image of 65/35 Time point 5 


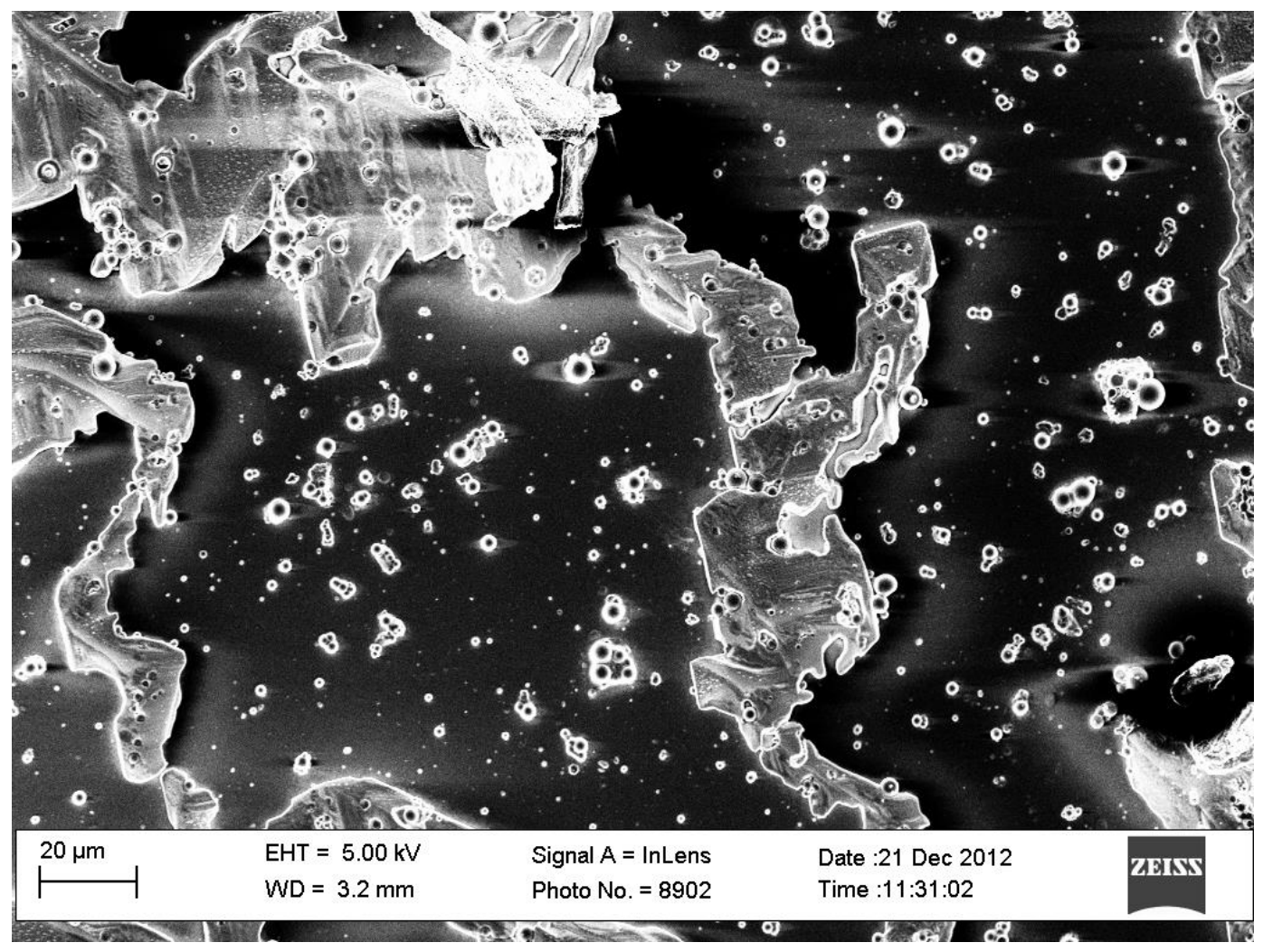

FIGURE 22-SEM image of 65/35 Time point 6 


\section{CONCLUSIONS}

FG/PLGA polymer mixtures were shown to be mixed more thoroughly through mechanical mixing via an overhead stirrer versus sonication. Due to issues of heating, water ingress, and variability of mixing outcomes, as seen through absorbance measurements, with sonication, mechanical mixing was determined to be a superior choice for FG/PGLA mixing. Further, degradation rates of varying relative concentrations of FG/PLGA were determined in a physiologic environment through treatment with PBS. Through this work, it has been proven that FG loses approximately $4 \%$ of its mass in the form of water when submitted to a heating process as mentioned previously in this document. Through GPC testing, it has also been shown that the molecular weight of FG is around $165,000 \mathrm{Da}$ which is very similar to cold water fish gelatin. The mixing of fish glue appears to be more thorough and with less variation at 1 hour of mechanical mixing time as compared to 30 minutes, 2 hours and 4 hours. The degradation rate of FG/PLGA mixtures at varying concentrations was successfully

measured showing a $70 \%$ drop within the first 12 hours of the exposure in a physiologically equivalent environment to the human body. 


\section{RECOMMENDATIONS}

If further research is to be pursued with a FG/PLGA mixture as a biomaterial for fiber fabrication, more studies need to be performed in fiber creation, cell growth and proliferation, and fiber degradation. First, an optimal relative concentration of FG/PLGA needs to be established with regards to fiber creation. The output of fiber diameter and ease of creation needs to be measured for varying relative concentrations of FG and PLGA. Once this is established, the ability for cells to grow and proliferate needs to be measured, imaged, and documented. Finally, the degradation rate of the fiber needs to be

measured as cells proliferate and grow around it, this test can also be done with or without attempting to provide fluid flow within the fabricated capillary's lumen. 


\section{REFERENCES}

Ayad, Shirley, Ray Boot-Handford, Martin Humphries, Karl Kadler, and Adrian Shuttleworth. 1998. The Extracellular Matrix Factsbook. 2nd ed.

Carbini, M, R Stevanato, M Rovea, P Traldi, and D Favretto. 1996. "Curie-Point Pyrolysis-Gas Chromatography/mass Spectrometry in the Art Field. 2--The Characterization of Proteinaceous Binders." Rapid Communications in Mass Spectrometry : RCM 10 (10): 1240-42. doi:10.1002/(SICI)10970231(19960731)10:10<1240::AID-RCM637>3.0.CO;2-X.

Grayson, Amy C R, Gabriela Voskerician, Aaron Lynn, James M Anderson, Michael J Cima, and Robert Langer. 2004. "Differential Degradation Rates in Vivo and in Vitro of Biocompatible Poly(lactic Acid) and Poly(glycolic Acid) Homo- and CoPolymers for a Polymeric Drug-Delivery Microchip." Journal of Biomaterials Science. Polymer Edition 15 (10). Netherlands: 1281-1304.

Gudmundsson, M. 2002. "Rheological Properties of Fish Gelatins." Journal of Food Science 67 (6): 2172-76. doi:10.1111/j.1365-2621.2002.tb09522.x.

Makadia, Hirenkumar K, and Steven J Siegel. 2011. "Poly Lactic-Co-Glycolic Acid (PLGA) as Biodegradable Controlled Drug Delivery Carrier.” Polymers 3 (3): 1377-97. doi:10.3390/polym3031377.

Muyonga, J.H, C.G.B Cole, and K.G Duodu. 2004. "Fourier Transform Infrared (FTIR) Spectroscopic Study of Acid Soluble Collagen and Gelatin from Skins and Bones of Young and Adult Nile Perch (Lates Niloticus)." Food Chemistry 86 (3): 325-32. doi:10.1016/j.foodchem.2003.09.038.

Muyonga, J.H., C.G.B. Cole, and K.G. Duodu. 2004. "Characterisation of Acid Soluble Collagen from Skins of Young and Adult Nile Perch (Lates Niloticus)." Food Chemistry 85 (1): 81-89. doi:10.1016/j.foodchem.2003.06.006.

Park, T G. 1995. "Degradation of Poly(lactic-Co-Glycolic Acid) Microspheres: Effect of Copolymer Composition.” Biomaterials 16 (15). ENGLAND: 1123-30.

Petukhova, Tatyana. 2000. “A History of Fish Glue as an Artist's Material: Applications in Paper and Parchment Artifacts." The Book and Paper Group Annual 19.

Turnay, J, and M D Ferna. 2002. "Structural and Physical Properties of Gelatin Extracted from Different Marine Species : A Comparative Study” 16: 25-34. 
Ulery, Bret D, Lakshmi S Nair, and Cato T Laurencin. 2011. "Biomedical Applications of Biodegradable Polymers." Journal of Polymer Science. Part B, Polymer Physics 49 (12): 832-64. doi:10.1002/polb.22259.

Zhang, Fengxiang, Shiying Xu, and Zhang Wang. 2011. "Pre-Treatment Optimization and Properties of Gelatin from Freshwater Fish Scales." Food and Bioproducts Processing 89 (3): 185-93. doi:10.1016/j.fbp.2010.05.003.

Gómez-Guillén, M.C., et al., Structural and physical properties of gelatin extracted from different marine species: a comparative study. Food Hydrocolloids, 2002. 16(1): p. 25-34.

An, K., et al., Preparation of fish gelatin and fish gelatin/poly(L-lactide) nanofibers by electrospinning. Int J Biol Macromol, 2010. 47(3): p. 380-8.

$\mathrm{Gu}, \mathrm{S} .-\mathrm{Y} .$, et al., Electrospinning of gelatin and gelatin/poly(l-lactide) blend and its characteristics for wound dressing. Materials Science and Engineering: C, 2009. 29(6): p. 1822-1828.

Barbani, N., et al., Bioartificial materials based on blends of collagen and poly(acrylic acid). Journal of Applied Polymer Science, 1999. 72(7): p. 971-976.

Coombes, A.G., et al., Biocomposites of non-crosslinked natural and synthetic polymers. Biomaterials, 2002. 23(10): p. 2113-8.

$\mathrm{Gu}, \mathrm{Z}$., et al., Synthesis and characterization of PLGA-gelatin complex with growth factor incorporation as potential matrix. Journal of Alloys and Compounds, 2009. 474(1-2): p. 450-454.

Jose, M.V., et al., Fabrication and characterization of aligned nanofibrous PLGA/Collagen blends as bone tissue scaffolds. Polymer, 2009. 50(15): p. 37783785 .

Li, X.K., et al., Characteristics of PLGA-gelatin complex as potential artificial nerve scaffold. Colloids Surf B Biointerfaces, 2007. 57(2): p. 198-203.

Berry, S.M., et al., Endothelial cell scaffolds generated by $3 D$ direct writing of biodegradable polymer microfibers. Biomaterials, 2011. 32(7): p. 1872-9. 


\section{CURRRICULUM VITA}

Born on January $2^{\text {nd }}, 1989$, in Edgewood, Kentucky to Philip Joseph and Mary Lynn Schworer, Adam Thomas Schworer has two older siblings: Andrew and Alex. During his undergraduate years, Adam spent multiple semesters on the Dean's list while serving as a Student Government Senator, performing Undergraduate Research under Dr. Robert Keynton, highlighted with part authorship credit on a manuscript published in the Journal of Biomaterials, and being active in his fraternity, Sigma Chi, most memorably as External Social Chair. Adam worked for three Co-op terms with Ethicon Endo Surgery Inc. a Johnson and Johnson Company in their Research and Development division. There, Adam gained experience on all stages of the New Product Development process for semi-disposable surgical instruments. Excelling in his first two co-op terms, Adam was appointed the Co-op Coordinator, by a committee of full-time employees, for his third term where he oversaw all Co-op related committees and activities. During his Graduate career, Adam studied the physiological processes of the body, cardiovascular dynamics, artificial organs, and the synthesis and characterization of biodegradable polymers. After completion of his Master's of Engineering in Bioengineering, Adam worked as an Associate Research and Development Engineer with Ethicon Inc a Johnson and Johnson Company in Blue Ash, Ohio for 18 months before returning to his Master's work. Adam looks forward to the prospect of a fruitful and fulfilling career in the development of technologies as he hopes to continues his education in law school. 


\section{APPENDICES}

\section{Appendix 1: Fish Glue Weight Analysis Raw Data}

\begin{tabular}{|c|c|c|c|c|c|c|c|}
\hline Specimen \# & Treatment & Slide Weight(mg) & $\begin{array}{l}\text { Wet Fish Glue } \\
\text { on Slide } \\
\text { Weight (mg) }\end{array}$ & $\begin{array}{l}\text { Wet FG } \\
\text { weight (mg) }\end{array}$ & $\begin{array}{l}\text { Dry Fish Glue on } \\
\text { Slide Weight (mg) }\end{array}$ & $\begin{array}{l}\text { Dry FG weight } \\
\text { (mg) }\end{array}$ & $\%$ Dry FG \\
\hline & 1 Heated & 41.08 & 70.15 & 29.07 & 57.67 & 16.59 & $57.07 \%$ \\
\hline & 2 Heated & 40.43 & 69.25 & 28.82 & 56.47 & 16.04 & $55.66 \%$ \\
\hline & 3 Heated & 40.96 & 68.47 & 27.51 & 56.86 & 15.9 & $57.80 \%$ \\
\hline & 4 Heated & 40.73 & 68.93 & 28.2 & 56.59 & 15.86 & $56.24 \%$ \\
\hline & 5 Heated & 40.73 & 71.17 & 30.44 & 58.25 & 17.52 & $57.56 \%$ \\
\hline & 6 No Heat & 41.01 & 66.27 & 25.26 & 54.36 & 13.35 & $52.85 \%$ \\
\hline & 7 No Heat & 40.62 & 68.08 & 27.46 & 54.93 & 14.31 & $52.11 \%$ \\
\hline & 8 No Heat & 41.83 & 69.73 & 27.9 & 56.59 & 14.76 & $52.90 \%$ \\
\hline & 9 No Heat & 40.84 & 63.86 & 23.02 & 52.99 & 12.15 & $52.78 \%$ \\
\hline & 10 No Heat & 41.18 & 68.75 & 27.57 & 55.75 & 14.57 & $52.85 \%$ \\
\hline
\end{tabular}


Appendix 2: Mixing Tests

\begin{tabular}{|c|c|c|c|c|c|c|}
\hline Mixing Type & Vial Number & Replicate & & 280 & Absorbance at 260 & $0 / 260$ \\
\hline Mechanical & & 1 & 1 & 1.702 & 2.849 & 0.597402597 \\
\hline Mechanical & & 1 & 2 & 1.292 & 2.413 & 0.535433071 \\
\hline Mechanical & & 1 & 3 & 1.122 & 2.225 & 0.504269663 \\
\hline Mechanical & & 2 & 1 & 0.931 & 2.083 & 0.446951512 \\
\hline Mechanical & & 2 & 2 & 1.123 & 2.33 & 0.481974249 \\
\hline Mechanical & & 2 & 3 & 1.056 & 2.225 & 0.474606742 \\
\hline Mechanical & & 3 & 1 & 1.984 & 3.144 & 0.631043257 \\
\hline Mechanical & & 3 & 2 & 1.987 & 2.893 & 0.68683028 \\
\hline Mechanical & & 3 & 3 & 1.879 & 3 & 0.626333333 \\
\hline Sonication & & 1 & 1 & 0.941 & 1.59 & 0.591823899 \\
\hline Sonication & & 1 & 2 & 0.915 & 1.54 & 0.594155844 \\
\hline Sonication & & 1 & 3 & 0.94 & 1.567 & 0.599872368 \\
\hline Sonication & & 2 & 1 & 1.211 & 1.998 & 0.606106106 \\
\hline Sonication & & 2 & 2 & 1.058 & 1.839 & 0.57531267 \\
\hline Sonication & & 2 & 3 & 1.11 & 2.243 & 0.494872938 \\
\hline Sonication & & 3 & 1 & 1.531 & 2.279 & 0.671785871 \\
\hline Sonication & & 3 & 2 & 1.476 & 2.236 & 0.660107335 \\
\hline Sonication & & 3 & 3 & 1.033 & 1.798 & 0.574527253 \\
\hline FG & & 1 & 1 & 1.57 & 2.478 & 0.633575464 \\
\hline FG & & 1 & 2 & 2.017 & 2.548 & 0.791601256 \\
\hline FG & & 1 & 3 & 1.554 & 2.462 & 0.631194151 \\
\hline PLGA & & 1 & 1 & 0.017 & 0.331 & 0.051359517 \\
\hline PLGA & & 1 & 2 & 0.035 & 0.256 & 0.13671875 \\
\hline PLGA & & 1 & 3 & 0.038 & 0.341 & 0.11143695 \\
\hline $30 \mathrm{~min}$ & & 1 & 1 & 1.321 & 2.494 & 0.529671211 \\
\hline $30 \mathrm{~min}$ & & 1 & 2 & 1.098 & 2.318 & 0.473684211 \\
\hline $30 \mathrm{~min}$ & & 1 & 3 & 0.439 & 1.271 & 0.345397325 \\
\hline $1 \mathrm{hr}$ & & 1 & 1 & 1.131 & 2.373 & 0.476611884 \\
\hline $1 \mathrm{hr}$ & & 1 & 2 & 1.016 & 2.248 & 0.451957295 \\
\hline $1 \mathrm{hr}$ & & 1 & 3 & 1.067 & 2.293 & 0.465329263 \\
\hline $2 \mathrm{hr}$ & & 1 & 1 & 1.22 & 2.497 & 0.488586304 \\
\hline $2 \mathrm{hr}$ & & 1 & 2 & 1.139 & 2.381 & 0.478370433 \\
\hline $2 \mathrm{hr}$ & & 1 & 3 & 1.139 & 2.381 & 0.478370433 \\
\hline
\end{tabular}


Appendix 3: Degradation Weight Analysis

\begin{tabular}{|c|c|c|c|c|c|c|c|c|}
\hline jecimen Specimen & $\begin{array}{l}\text { Time } \\
\text { Point }\end{array}$ & & $\begin{array}{l}\text { Cover } \\
\text { Glass }\end{array}$ & $\begin{array}{l}\text { Initial Fish } \\
\text { Glue }+ \\
\text { Coverglass }\end{array}$ & $\begin{array}{l}\text { Final Fish } \\
\text { Glue }+ \\
\text { Coverglass }\end{array}$ & $\begin{array}{l}\text { Initial } \\
\text { Fish } \\
\text { Glue }\end{array}$ & $\begin{array}{l}\text { Final } \\
\text { Fish } \\
\text { Glue }\end{array}$ & $\begin{array}{l}\text { Percent } \\
\text { Weight }\end{array}$ \\
\hline Number Type & (Days) & Replicate & Weight & Weight & Weight & Weight & Weight & Remaining \\
\hline $4965 / 35$ & 0.5 & 1 & 40.98 & 46.54 & 43.31 & 5.56 & 2.33 & 41.91 \\
\hline $5065 / 35$ & 0.5 & 2 & 40.91 & 47.32 & 42.34 & 6.41 & 1.43 & 22.31 \\
\hline $5165 / 35$ & 0.5 & 3 & 41.02 & 45.18 & 42.12 & 4.16 & 1.1 & 26.44 \\
\hline $5570 / 30$ & 0.5 & 1 & 41.12 & 48.87 & 42.58 & 7.75 & 1.46 & 18.84 \\
\hline $5670 / 30$ & 0.5 & 2 & 40.87 & 47.79 & 43.36 & 6.92 & 2.49 & 35.98 \\
\hline $5770 / 30$ & 0.5 & 3 & 40.85 & 46.92 & 42.4 & 6.07 & 1.55 & 25.54 \\
\hline $6175 / 25$ & 0.5 & 1 & 40.78 & 52.21 & 44.51 & 11.43 & 3.73 & 32.63 \\
\hline $6275 / 25$ & 0.5 & 2 & 40.87 & 47.34 & 42.34 & 6.47 & 1.47 & 22.72 \\
\hline $6375 / 25$ & 0.5 & 3 & 40.68 & 46.98 & 41.98 & 6.3 & 1.3 & 20.63 \\
\hline $6780 / 20$ & 0.5 & 1 & 41.31 & 51.38 & 43.05 & 10.07 & 1.74 & 17.28 \\
\hline $6880 / 20$ & 0.5 & 2 & 40.77 & 50.13 & 43.86 & 9.36 & 3.09 & 33.01 \\
\hline $6980 / 20$ & 0.5 & 3 & 40.67 & 48.23 & 42.12 & 7.56 & 1.45 & 19.18 \\
\hline $5265 / 35$ & 1 & 1 & 41.28 & 47.18 & 42.1 & 5.9 & 0.82 & 13.90 \\
\hline $5365 / 35$ & 1 & 2 & 40.47 & 44.31 & 41.21 & 3.84 & 0.74 & 19.27 \\
\hline $5465 / 35$ & 1 & 3 & 40.91 & 45.73 & 42.12 & 4.82 & 1.21 & 25.10 \\
\hline $5870 / 30$ & 1 & 1 & 41.17 & 48.34 & 42.9 & 7.17 & 1.73 & 24.13 \\
\hline $5970 / 30$ & 1 & 2 & 41.53 & 52.1 & 43.18 & 10.57 & 1.65 & 15.61 \\
\hline $6070 / 30$ & 1 & 3 & 41.33 & 49.32 & 42.2 & 7.99 & 0.87 & 10.89 \\
\hline $6475 / 25$ & 1 & 1 & 40.9 & 47.92 & 41.86 & 7.02 & 0.96 & 13.68 \\
\hline $6575 / 25$ & 1 & 2 & 41.02 & 48.28 & 41.98 & 7.26 & 0.96 & 13.22 \\
\hline $6675 / 25$ & 1 & 3 & 41.04 & 51.76 & 41.72 & 10.72 & 0.68 & 6.34 \\
\hline $7080 / 20$ & 1 & 1 & 41.12 & 47.87 & 42.19 & 6.75 & 1.07 & 15.85 \\
\hline $7180 / 20$ & 1 & 2 & 40.99 & 50.87 & 41.72 & 9.88 & 0.73 & 7.39 \\
\hline $7280 / 20$ & 1 & 3 & 41.43 & 51.03 & 41.99 & 9.6 & 0.56 & 5.83 \\
\hline $165 / 35$ & 2 & 1 & 40.82 & 46.64 & 41.49 & 5.82 & 0.67 & 11.51 \\
\hline $265 / 35$ & 2 & 2 & 40.91 & 46.12 & 41.22 & 5.21 & 0.31 & 5.95 \\
\hline $365 / 35$ & 2 & 3 & 41.14 & 45.95 & 41.72 & 4.81 & 0.58 & 12.06 \\
\hline $1370 / 30$ & 2 & 1 & 40.64 & 49.38 & 41.2 & 8.74 & 0.56 & 6.41 \\
\hline $1470 / 30$ & 2 & 2 & 41.2 & 49.4 & 41.89 & 8.2 & 0.69 & 8.41 \\
\hline $1570 / 30$ & 2 & 3 & 40.45 & 49.04 & 40.84 & 8.59 & 0.39 & 4.54 \\
\hline $2575 / 25$ & 2 & 1 & 41.11 & 51.69 & 41.39 & 10.58 & 0.28 & 2.65 \\
\hline
\end{tabular}




\begin{tabular}{|c|c|c|c|c|c|c|c|c|}
\hline $2675 / 25$ & 2 & 2 & 40.5 & 50.89 & 41.18 & 10.39 & 0.68 & 6.54 \\
\hline $2775 / 25$ & 2 & 3 & 41.18 & 51.3 & 41.37 & 10.12 & 0.19 & 1.88 \\
\hline $3780 / 20$ & 2 & 1 & 40.91 & 45.47 & 41.23 & 4.56 & 0.32 & 7.02 \\
\hline $3880 / 20$ & 2 & 2 & 40.81 & 44.88 & 41.08 & 4.07 & 0.27 & 6.63 \\
\hline $3980 / 20$ & 2 & 3 & 41.39 & 46.24 & 41.52 & 4.85 & 0.13 & 2.68 \\
\hline $465 / 35$ & 6 & 1 & 41.48 & 49.69 & 41.93 & 8.21 & 0.45 & 5.48 \\
\hline $565 / 35$ & 6 & 2 & 40.65 & 49.29 & 41.24 & 8.64 & 0.59 & 6.83 \\
\hline $665 / 35$ & 6 & 3 & 41.12 & 46.92 & 41.42 & 5.8 & 0.3 & 5.17 \\
\hline $1670 / 30$ & 6 & 1 & 40.92 & 49.37 & 41.57 & 8.45 & 0.65 & 7.69 \\
\hline $1770 / 30$ & 6 & 2 & 40.79 & 49.39 & 41.3 & 8.6 & 0.51 & 5.93 \\
\hline $1870 / 30$ & 6 & 3 & 41.44 & 49.77 & 41.7 & 8.33 & 0.26 & 3.12 \\
\hline $2875 / 25$ & 6 & 1 & 40.62 & 50.23 & 40.82 & 9.61 & 0.2 & 2.08 \\
\hline $2975 / 25$ & 6 & 2 & 41.38 & 49.44 & 41.53 & 8.06 & 0.15 & 1.86 \\
\hline $3075 / 25$ & 6 & 3 & 40.98 & 51.37 & 41.16 & 10.39 & 0.18 & 1.73 \\
\hline $4080 / 20$ & 6 & 1 & 40.98 & 45.11 & 41.13 & 4.13 & 0.15 & 3.63 \\
\hline $4180 / 20$ & 6 & 2 & 40.95 & 45.33 & 41.08 & 4.38 & 0.13 & 2.97 \\
\hline $4280 / 20$ & 6 & 3 & 41 & 46.91 & 41.07 & 5.91 & 0.07 & 1.18 \\
\hline $765 / 35$ & 10 & 1 & 40.76 & 47.05 & 41.49 & 6.29 & 0.73 & 11.61 \\
\hline $865 / 35$ & 10 & 2 & 40.98 & 46.56 & 41.32 & 5.58 & 0.34 & 6.09 \\
\hline $965 / 35$ & 10 & 3 & 40.63 & 45.09 & 40.99 & 4.46 & 0.36 & 8.07 \\
\hline $1970 / 30$ & 10 & 1 & 40.97 & 49.18 & 41.23 & 8.21 & 0.26 & 3.17 \\
\hline $2070 / 30$ & 10 & 2 & 40.58 & 48.68 & 40.95 & 8.1 & 0.37 & 4.57 \\
\hline $2170 / 30$ & 10 & 3 & 40.67 & 46.67 & 41.04 & 6 & 0.37 & 6.17 \\
\hline $3175 / 25$ & 10 & 1 & 41.1 & 49.21 & 41.46 & 8.11 & 0.36 & 4.44 \\
\hline $3275 / 25$ & 10 & 2 & 40.82 & 50.77 & 41.42 & 9.95 & 0.6 & 6.03 \\
\hline $3375 / 25$ & 10 & 3 & 40.96 & 50.55 & 41.45 & 9.59 & 0.49 & 5.11 \\
\hline $4380 / 20$ & 10 & 1 & 40.75 & 46.2 & 40.91 & 5.45 & 0.16 & 2.94 \\
\hline $4480 / 20$ & 10 & 2 & 40.62 & 45.81 & 40.77 & 5.19 & 0.15 & 2.89 \\
\hline $4580 / 20$ & 10 & 3 & 40.61 & 44.8 & 40.75 & 4.19 & 0.14 & 3.34 \\
\hline $1065 / 35$ & 14 & 1 & 40.98 & 46.77 & 41.13 & 5.79 & 0.15 & 2.59 \\
\hline $1165 / 35$ & 14 & 2 & 41.03 & 46.82 & 41.38 & 5.79 & 0.35 & 6.04 \\
\hline $1265 / 35$ & 14 & 3 & 40.77 & 47.43 & 41.35 & 6.66 & 0.58 & 8.71 \\
\hline $2270 / 30$ & 14 & 1 & 40.73 & 49.43 & 40.96 & 8.7 & 0.23 & 2.64 \\
\hline $2370 / 30$ & 14 & 2 & 40.74 & 50.18 & 41.12 & 9.44 & 0.38 & 4.03 \\
\hline $2470 / 30$ & 14 & 3 & 40.69 & 48.49 & 41.23 & 7.8 & 0.54 & 6.92 \\
\hline $3475 / 25$ & 14 & 1 & 40.46 & 49.12 & 40.83 & 8.66 & 0.37 & 4.27 \\
\hline $3575 / 25$ & 14 & 2 & 40.91 & 51.01 & 41.13 & 10.1 & 0.22 & 2.18 \\
\hline $3675 / 25$ & 14 & 3 & 41.54 & 53.52 & 41.91 & 11.98 & 0.37 & 3.09 \\
\hline
\end{tabular}




$\begin{array}{rrrrrrrrr}4680 / 20 & 14 & 1 & 40.51 & 44.69 & 40.78 & 4.18 & 0.27 & 6.46 \\ 4780 / 20 & 14 & 2 & 40.58 & 44.47 & 40.68 & 3.89 & 0.1 & 2.57 \\ 4880 / 20 & 14 & 3 & 40.84 & 46.16 & 41.13 & 5.32 & 0.29 & 5.45\end{array}$


Appendix 4: SEM images of mixtures

6535_TP0_1

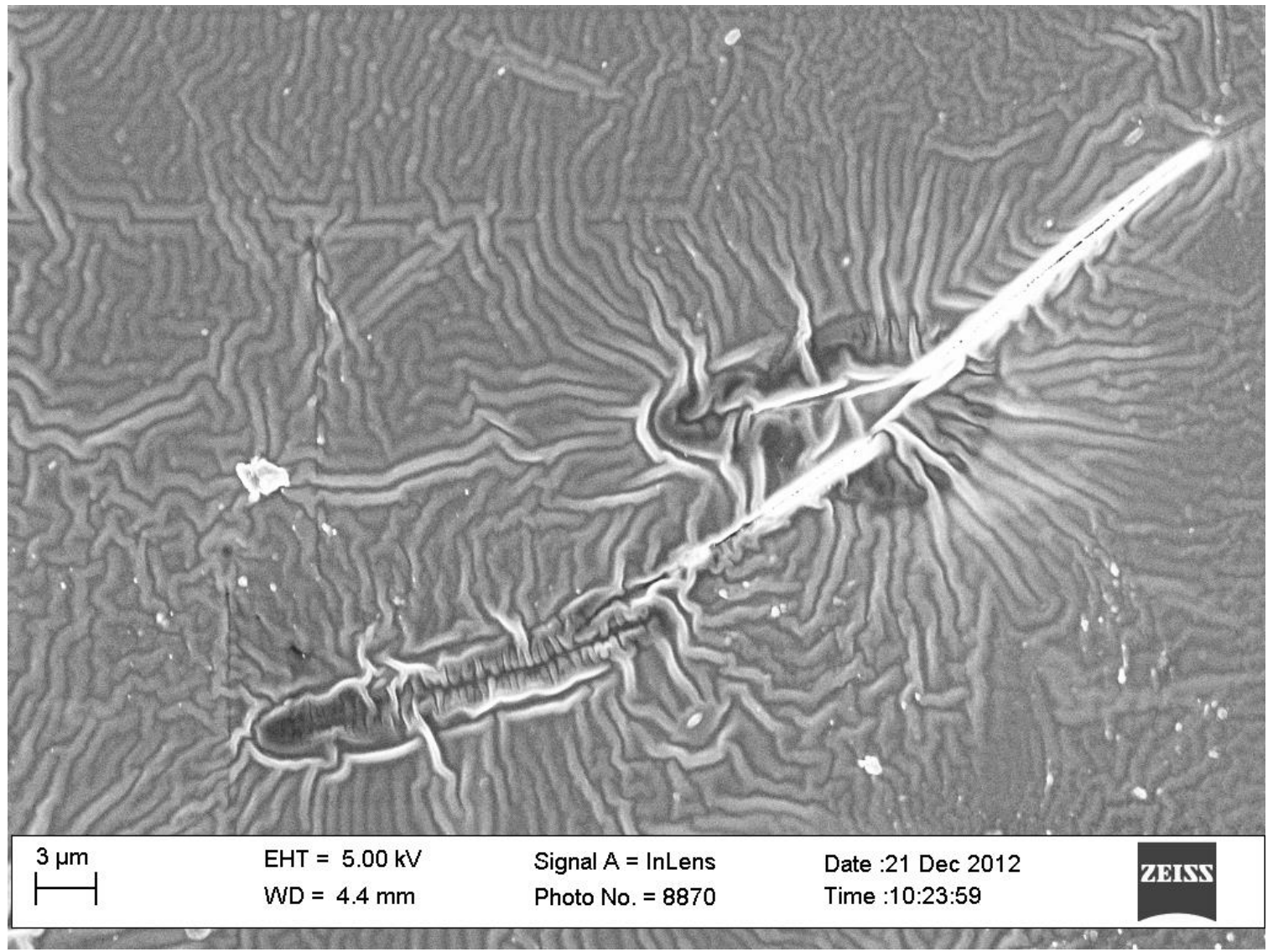

6535_TP0_2 


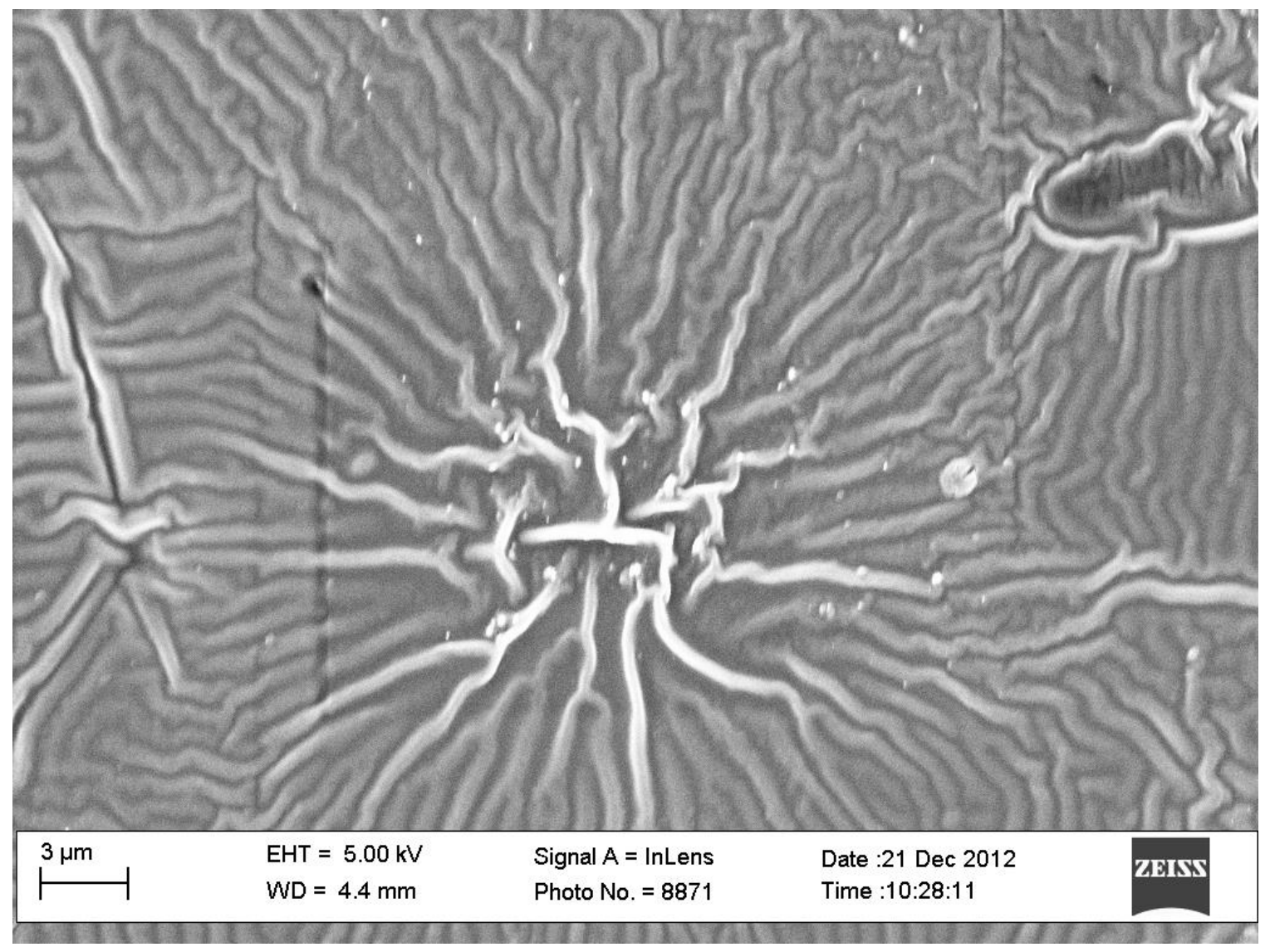

6535_TP0_3 


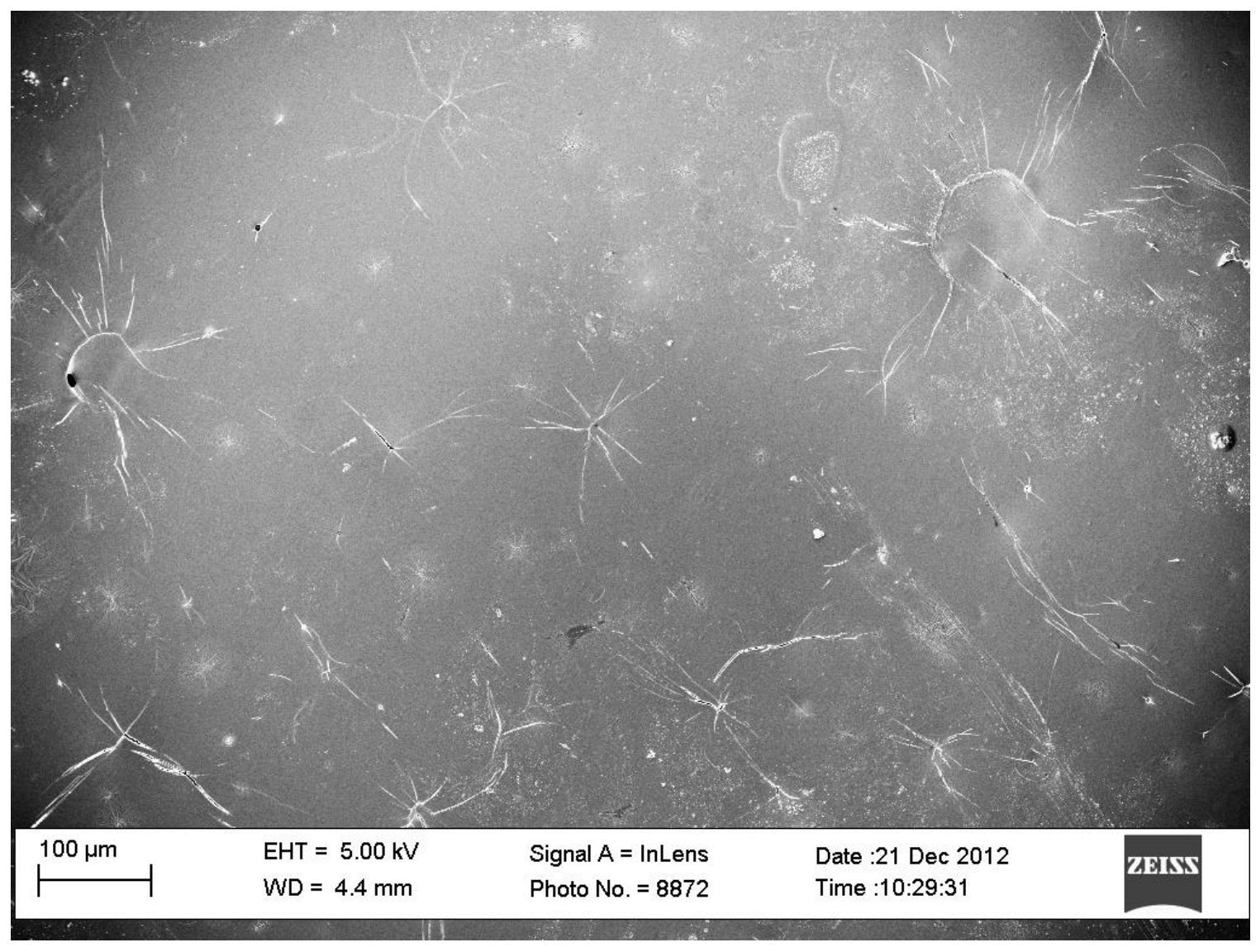




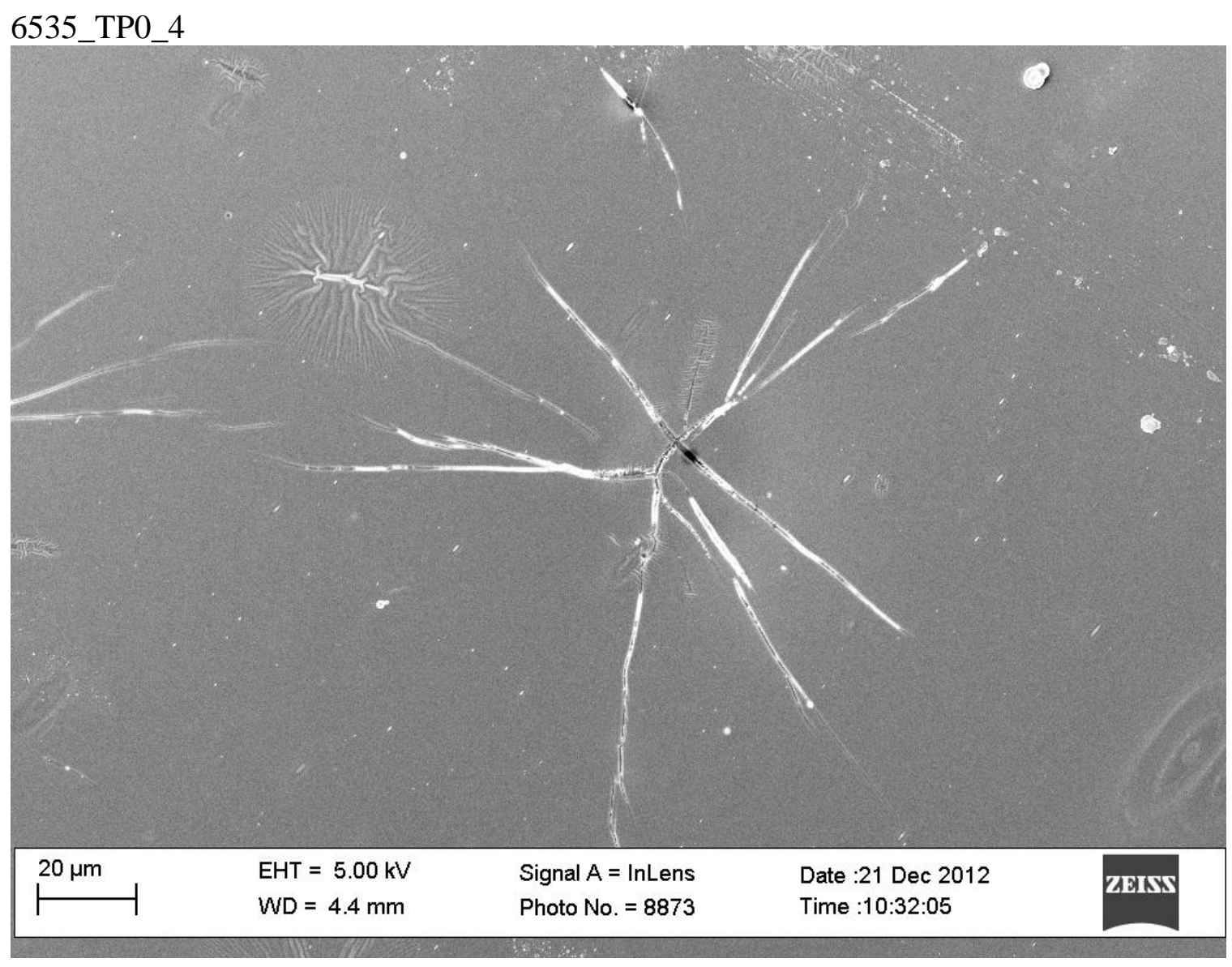




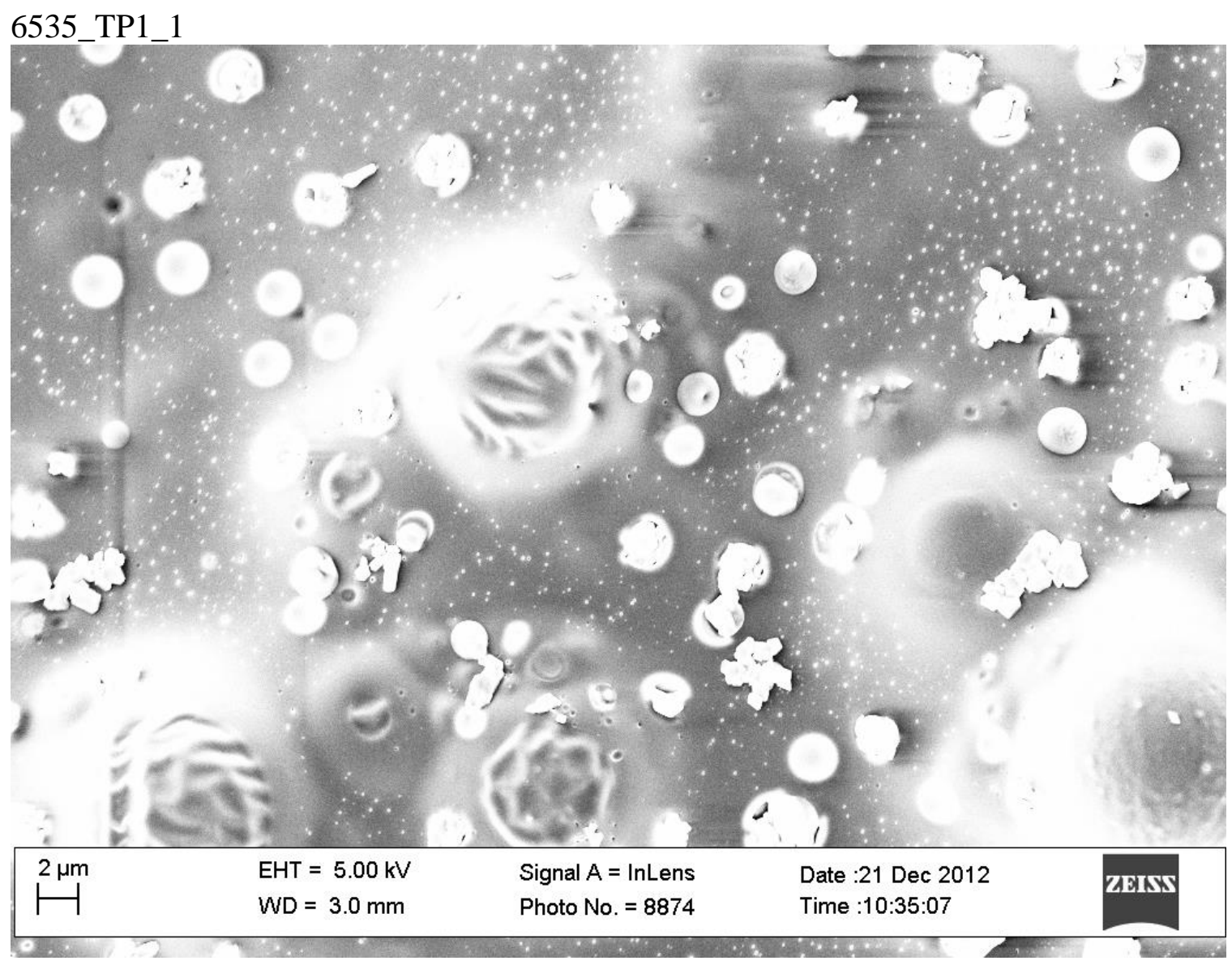




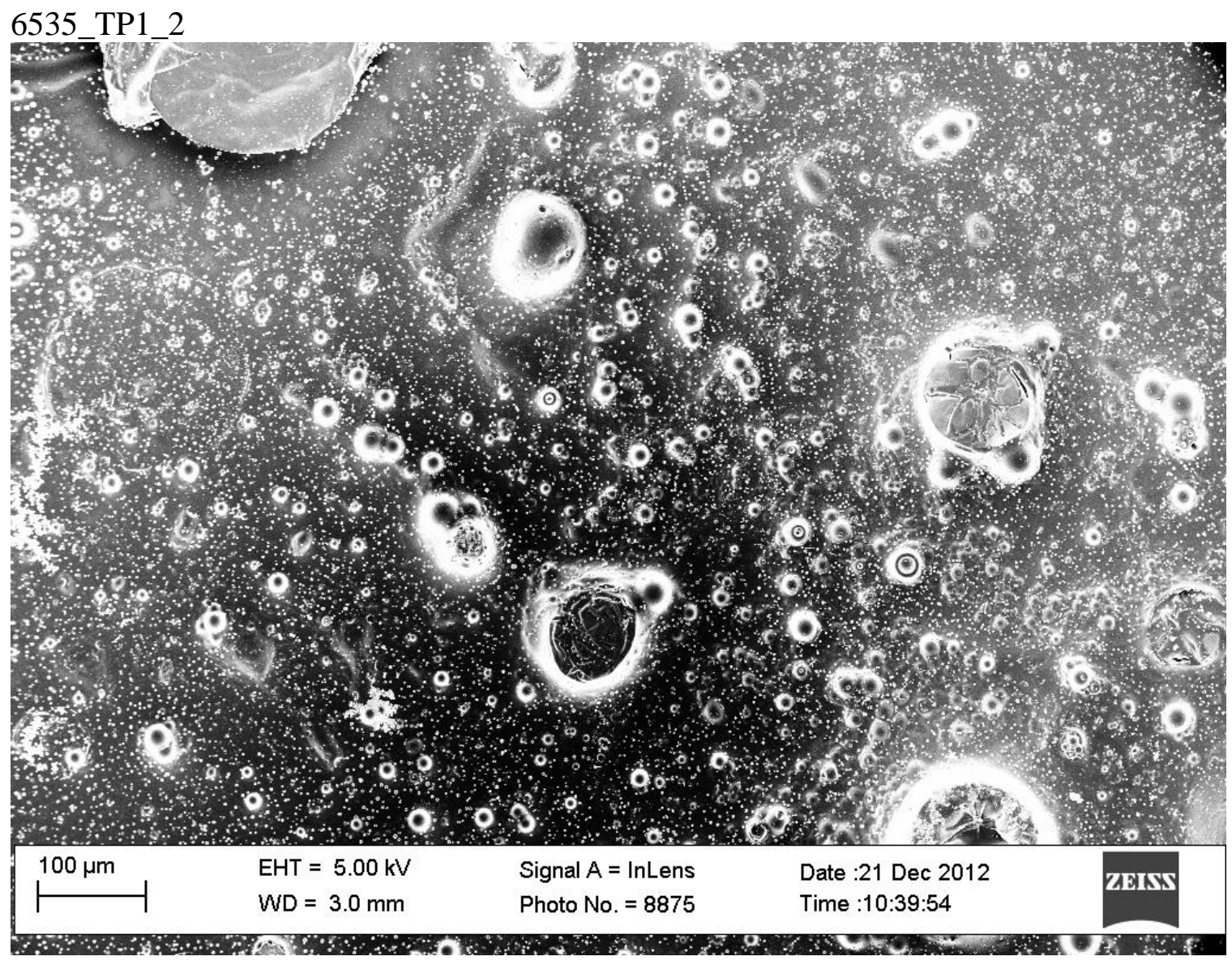




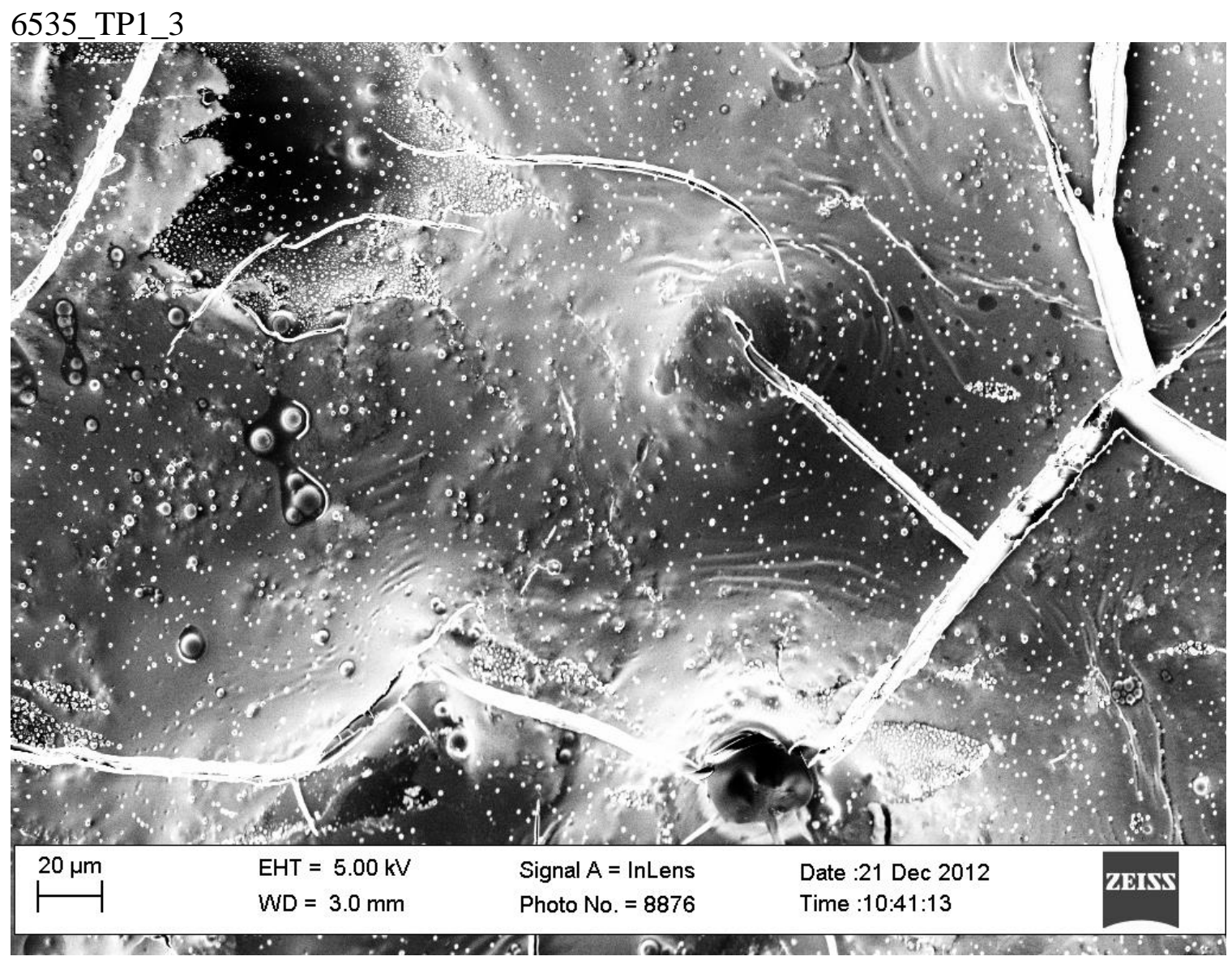




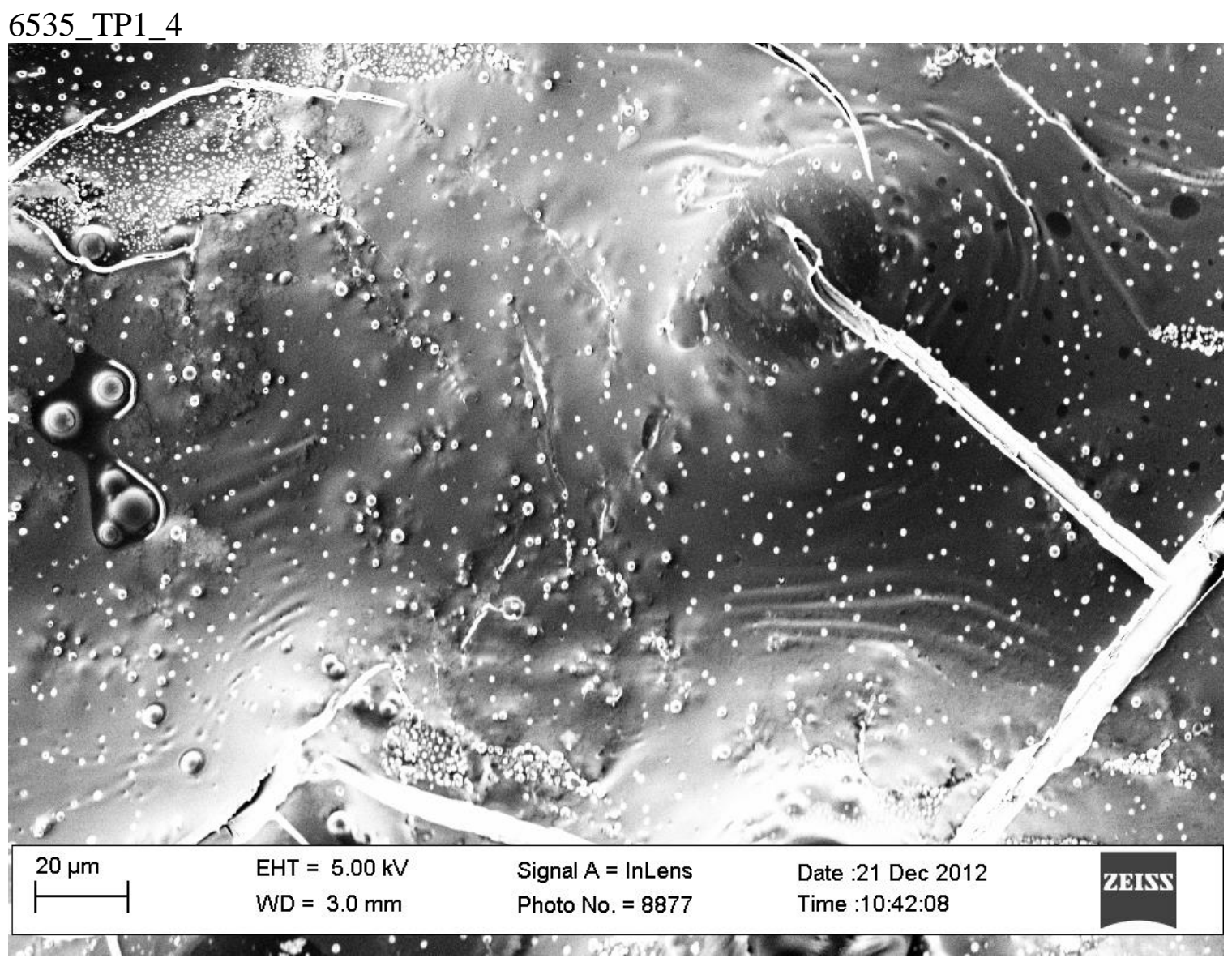




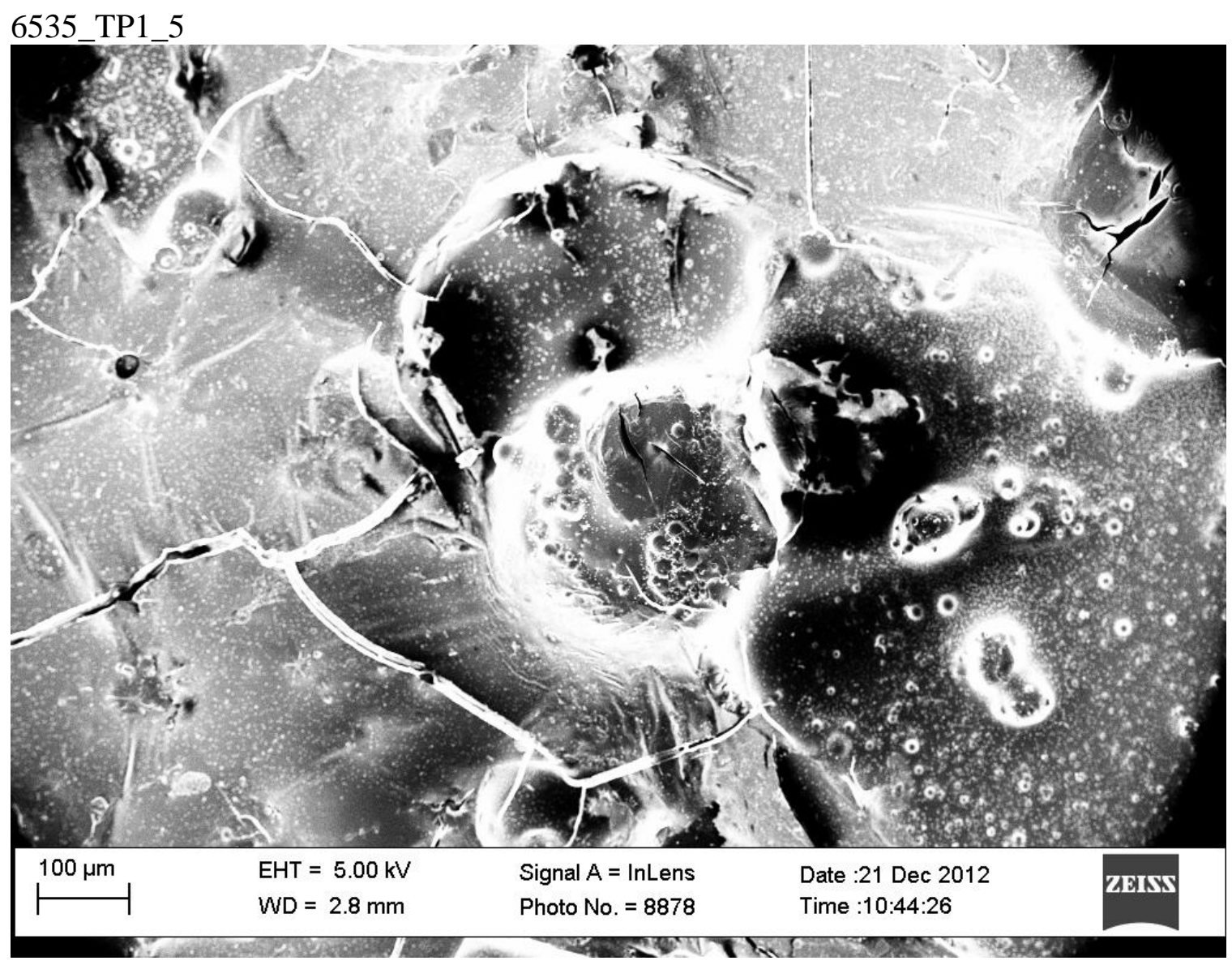




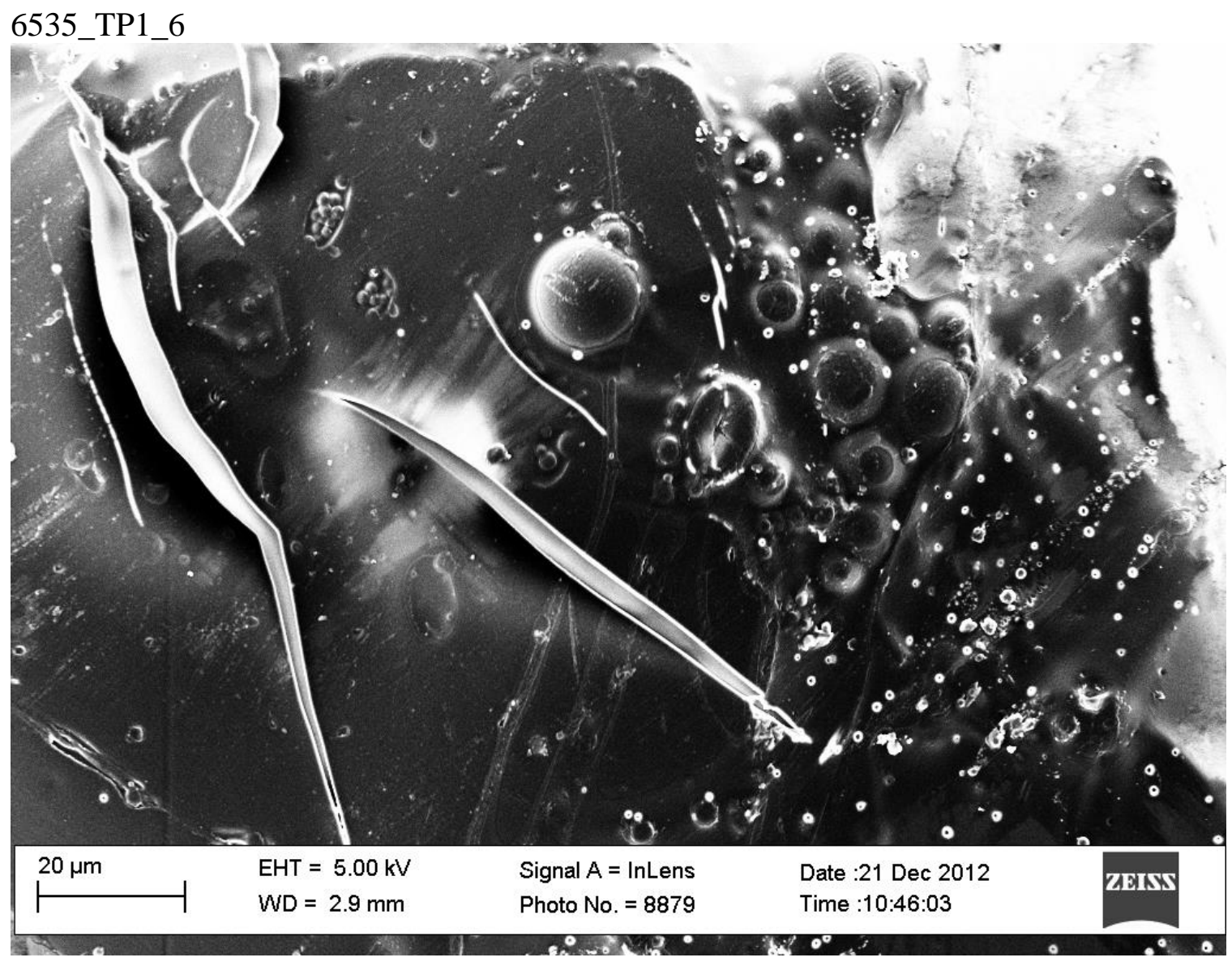




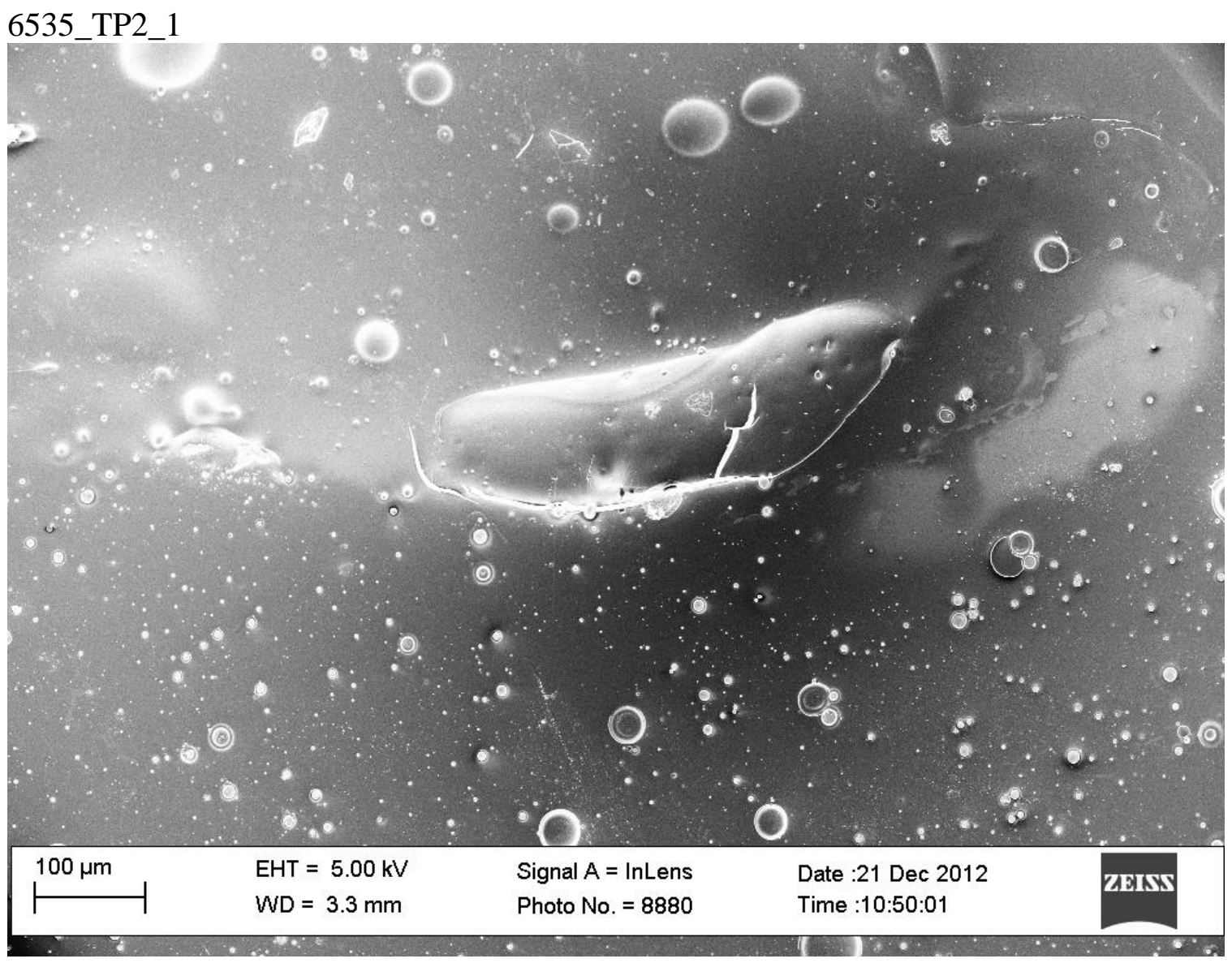




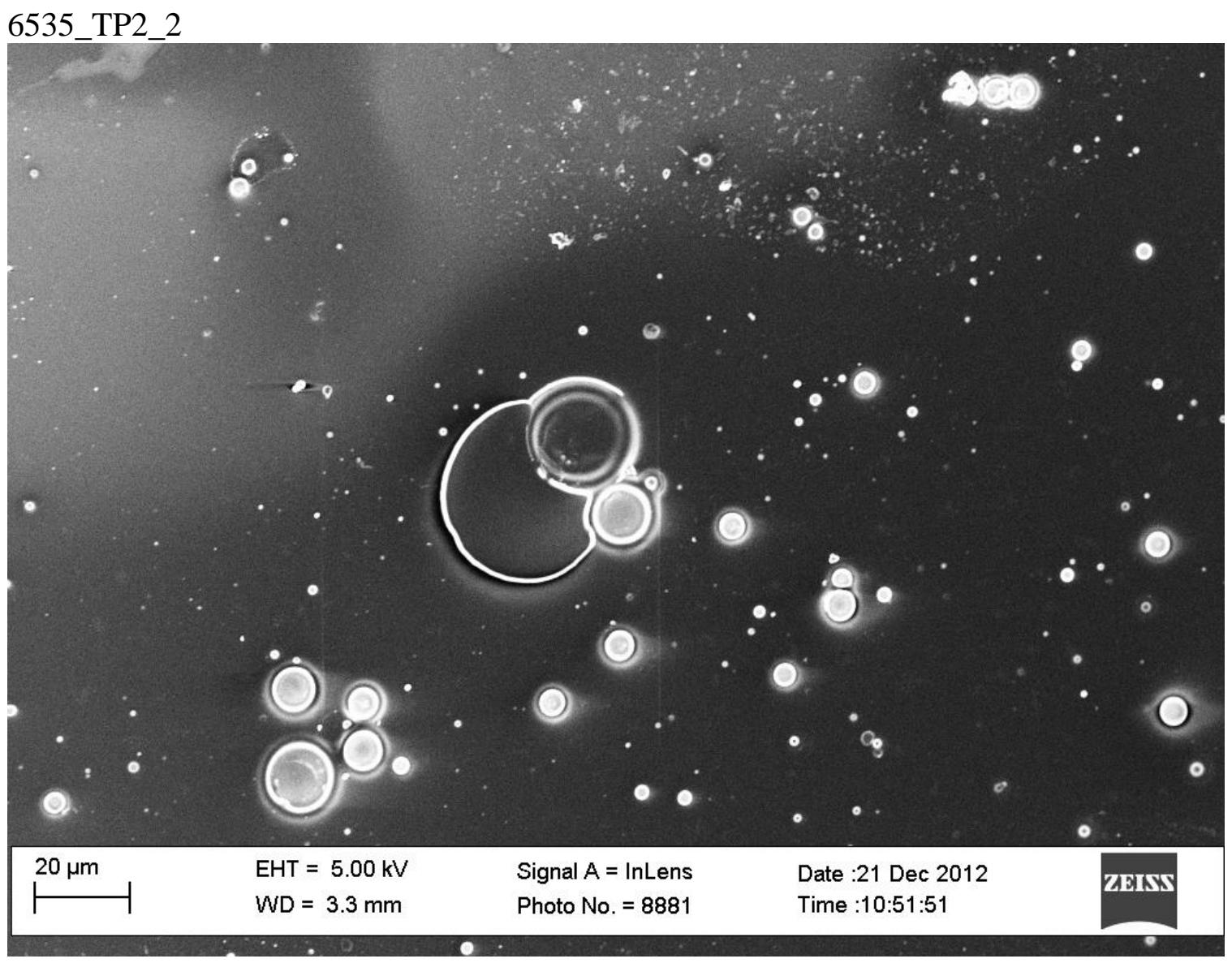




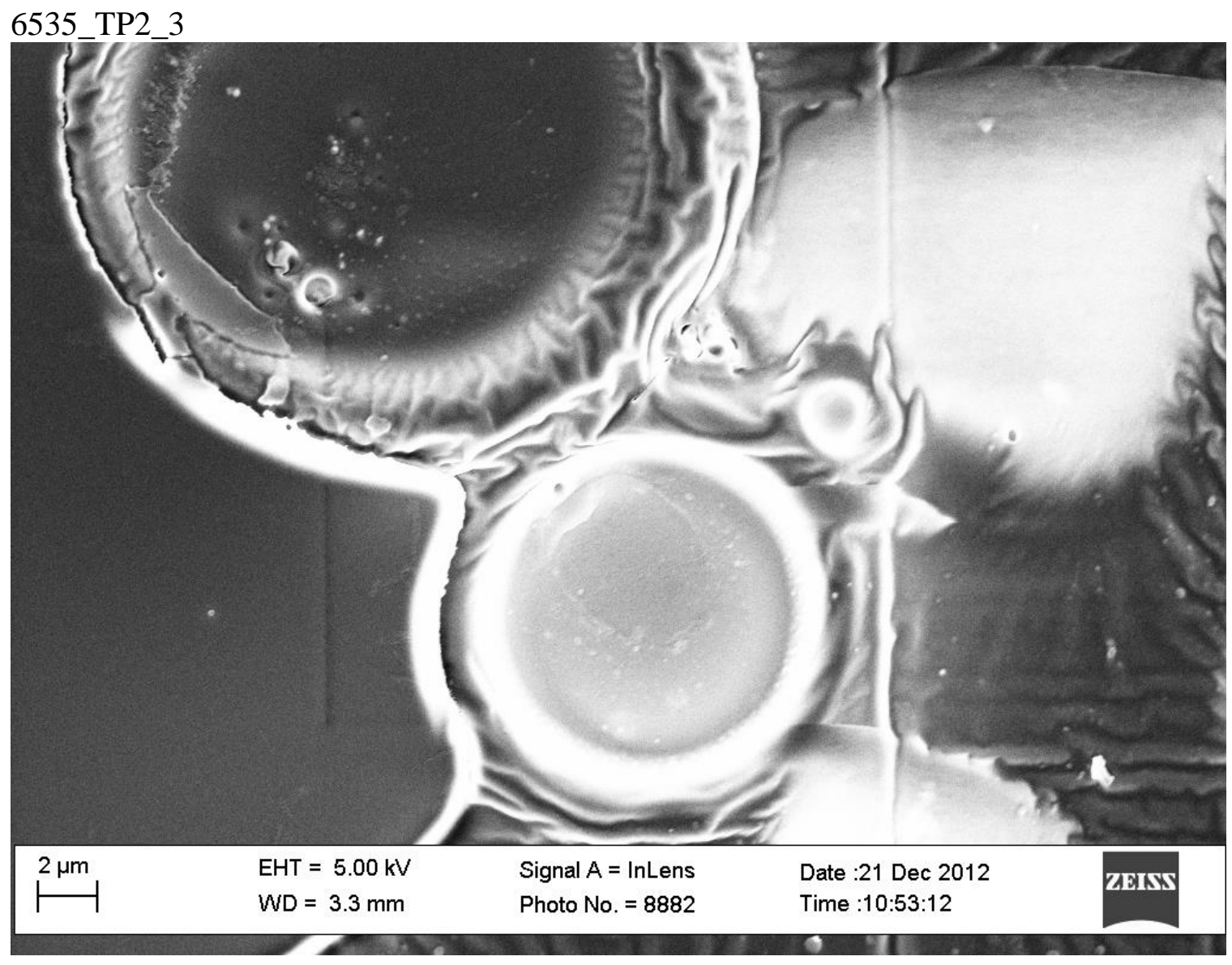




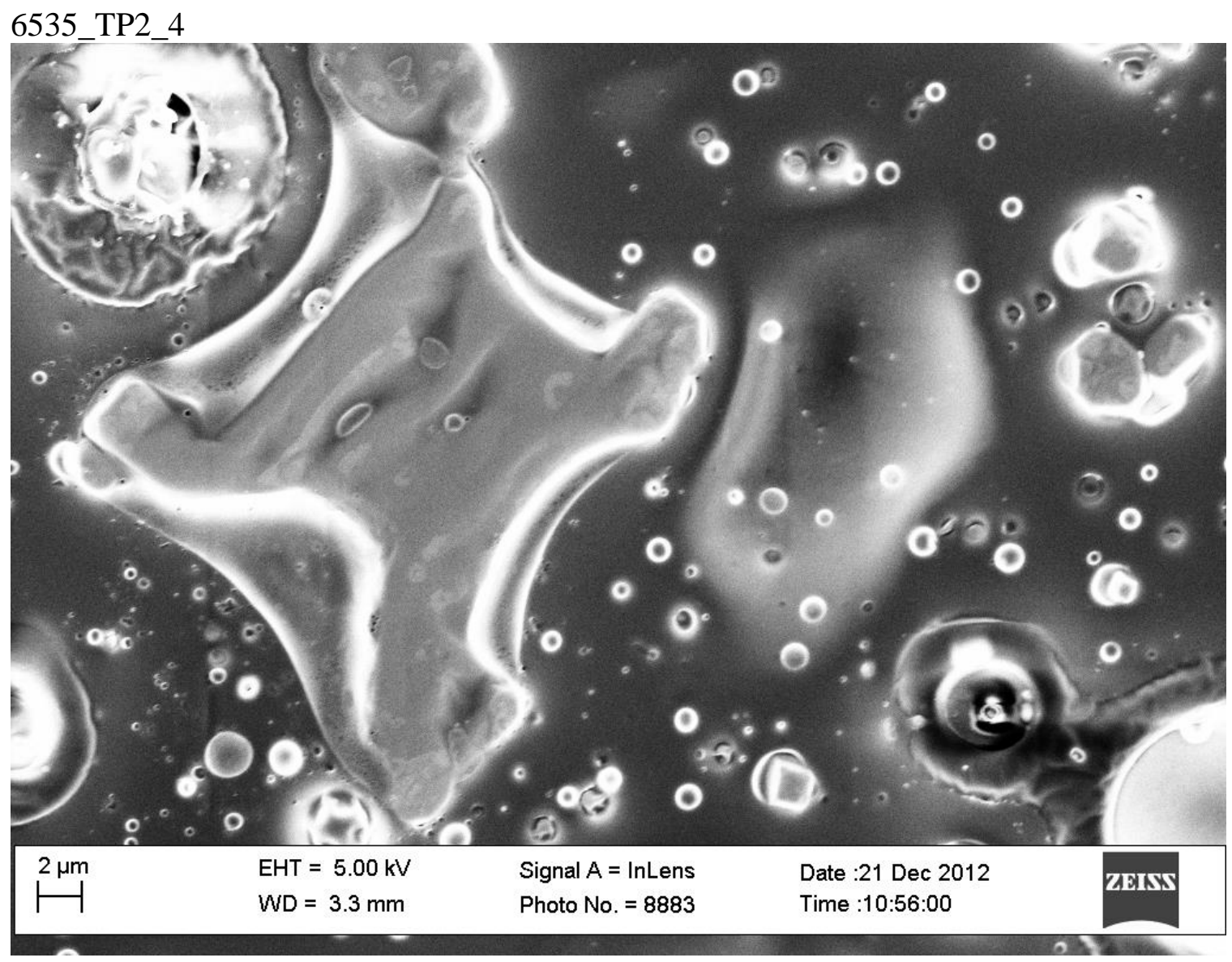




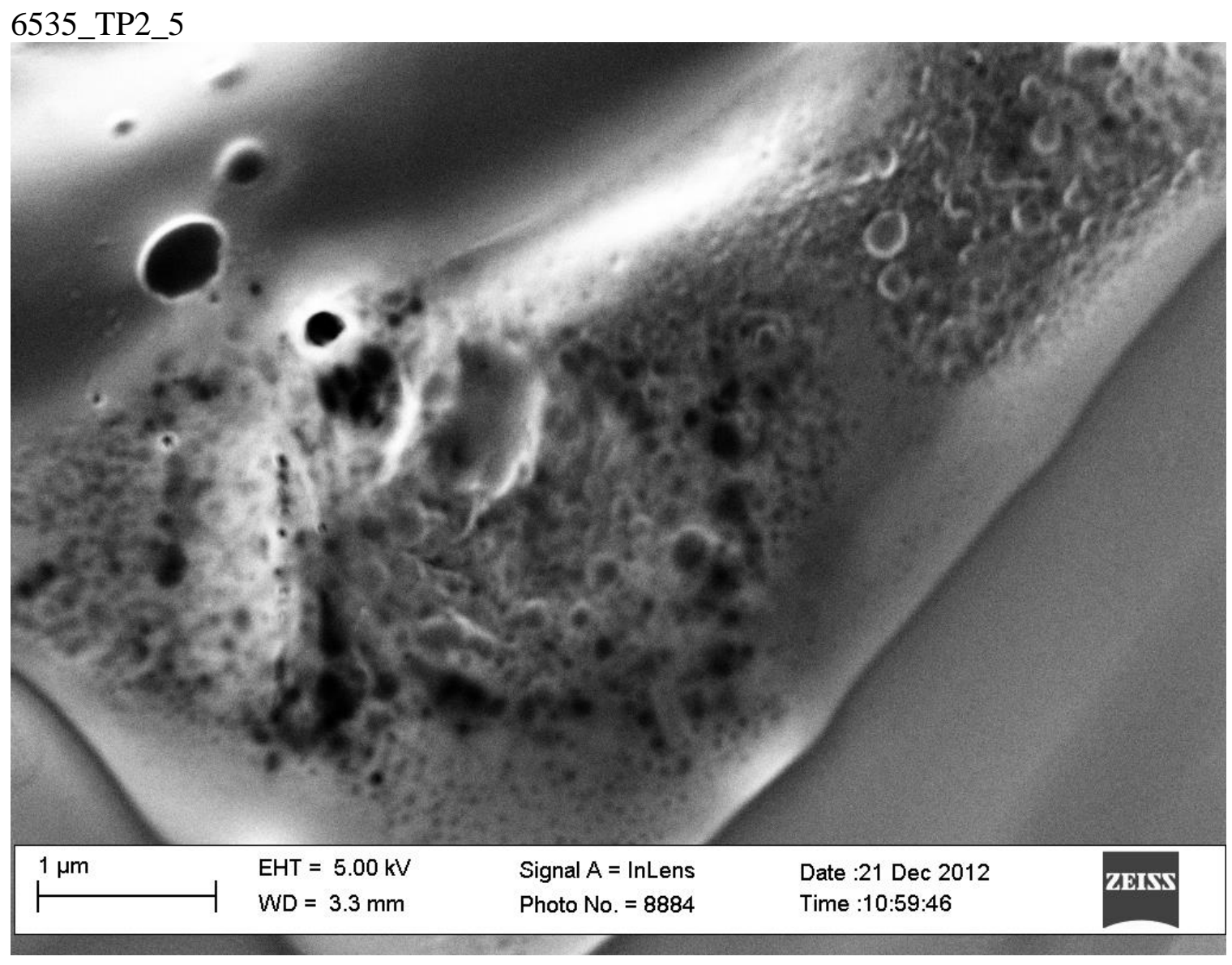




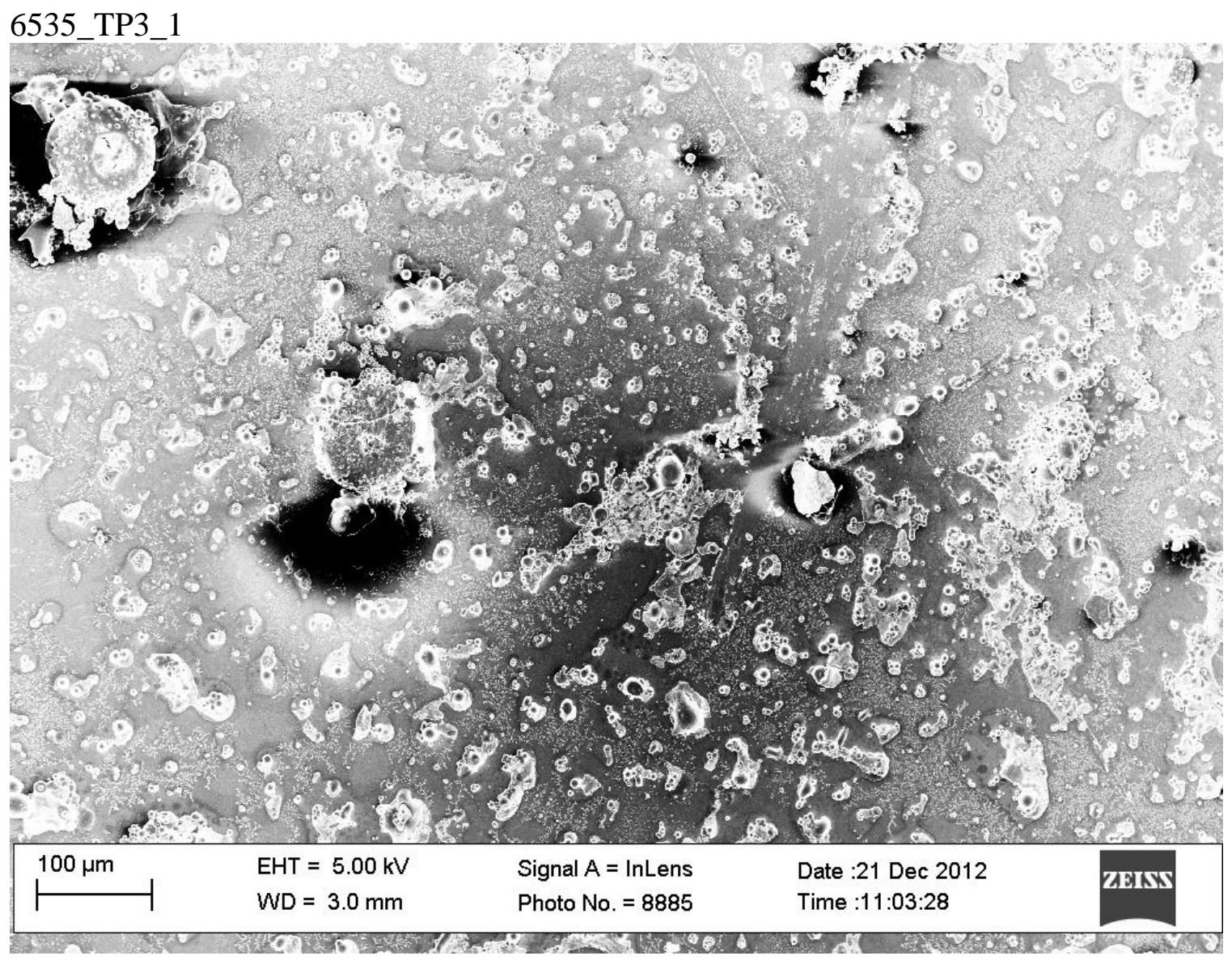




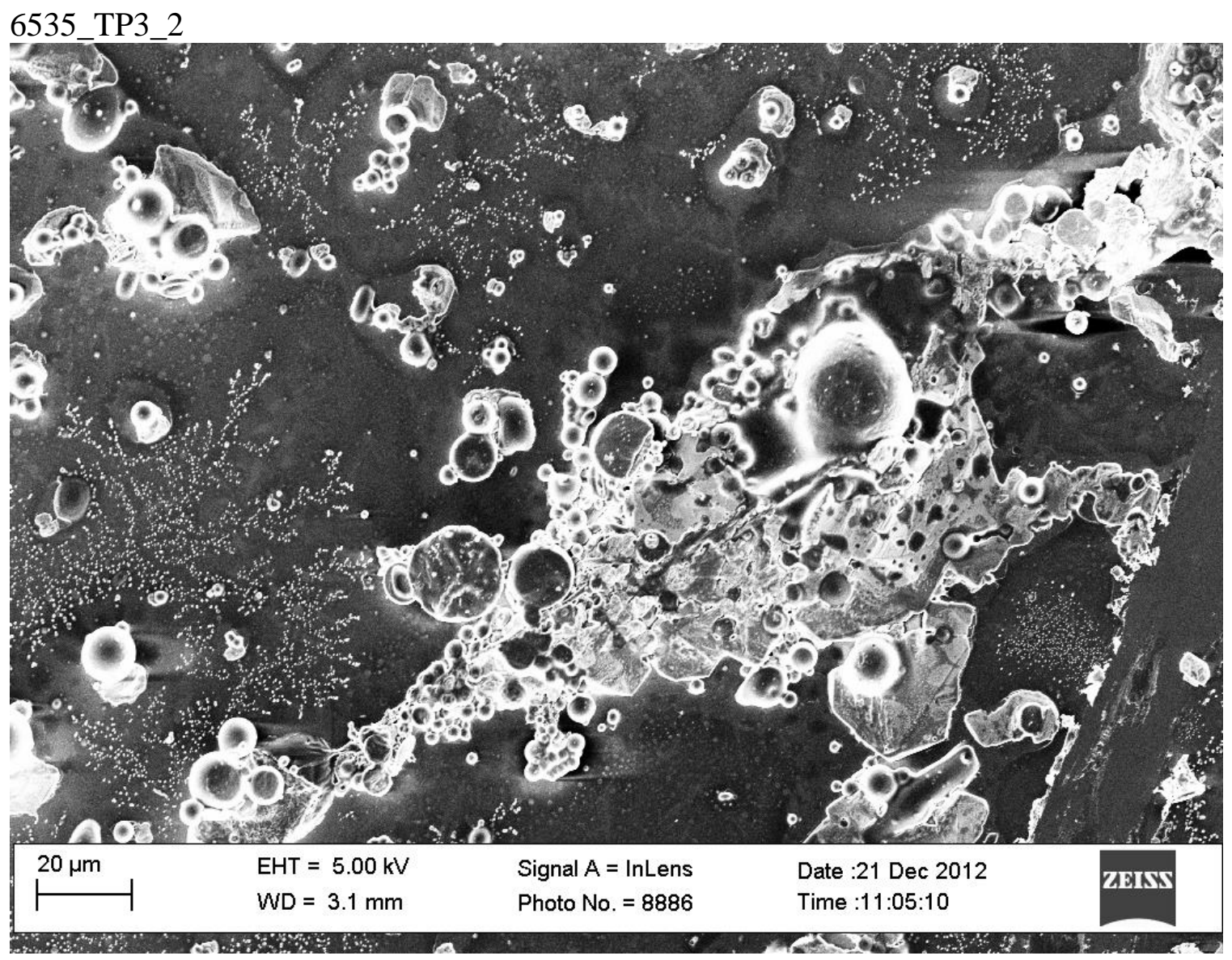


6535_TP3_3

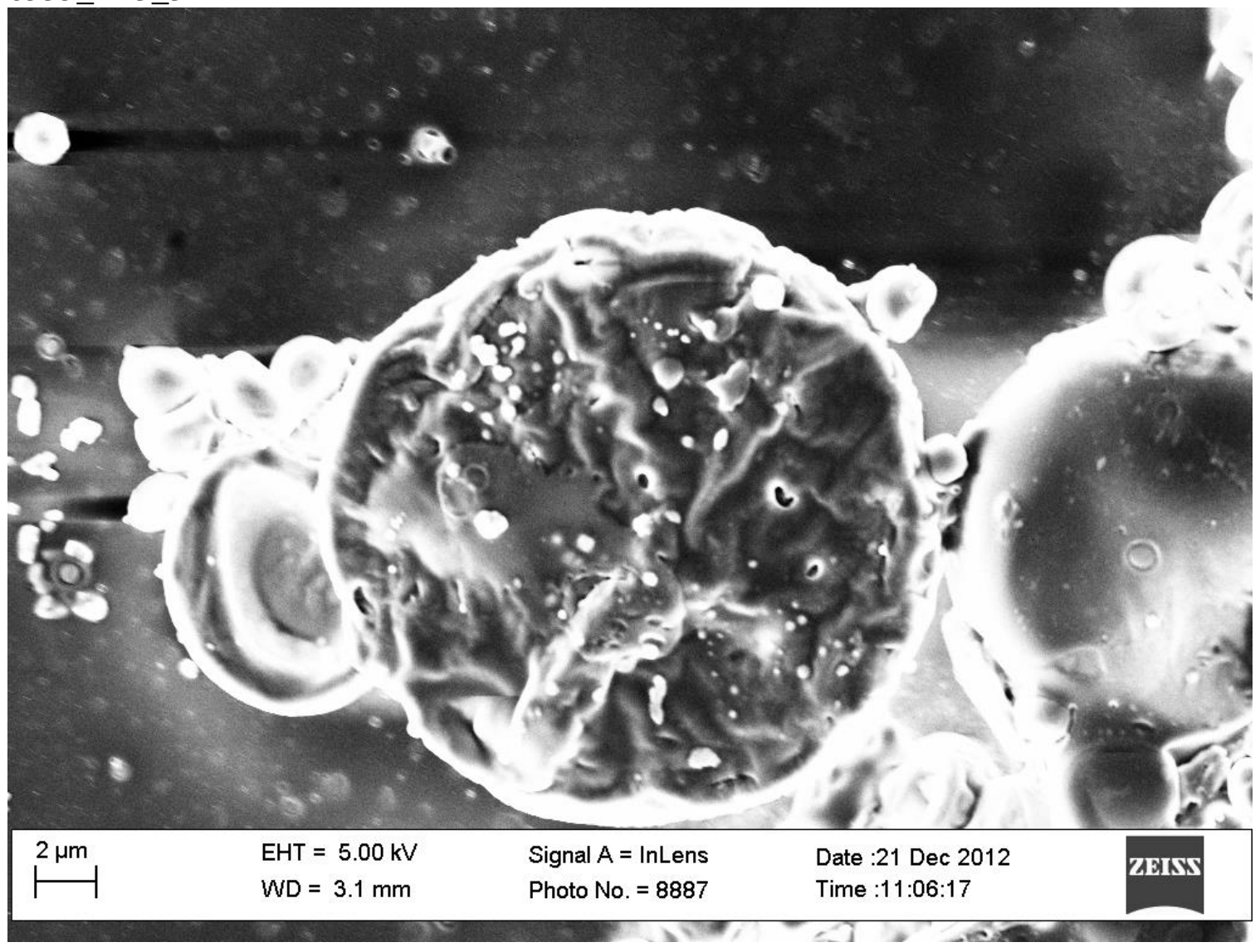




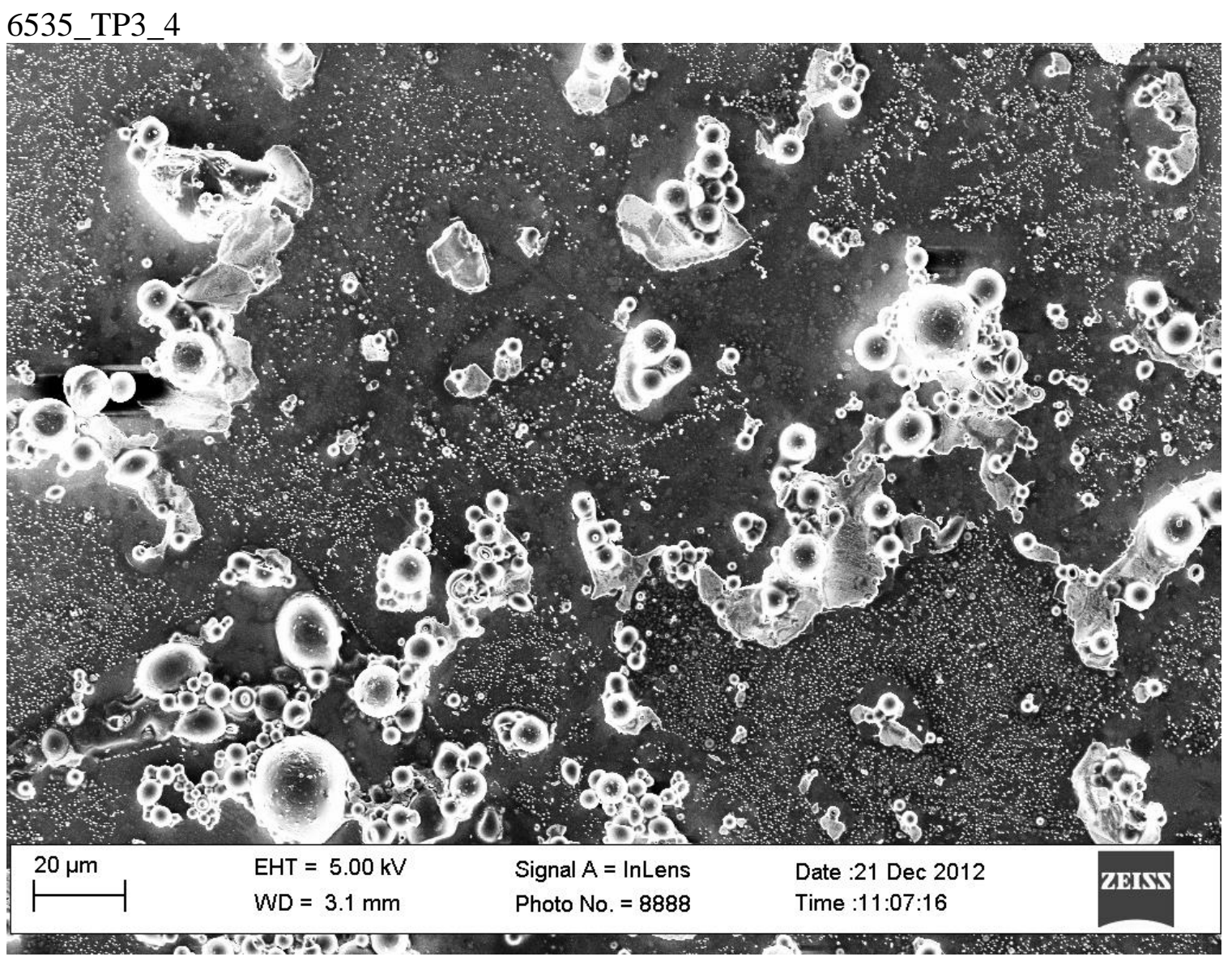




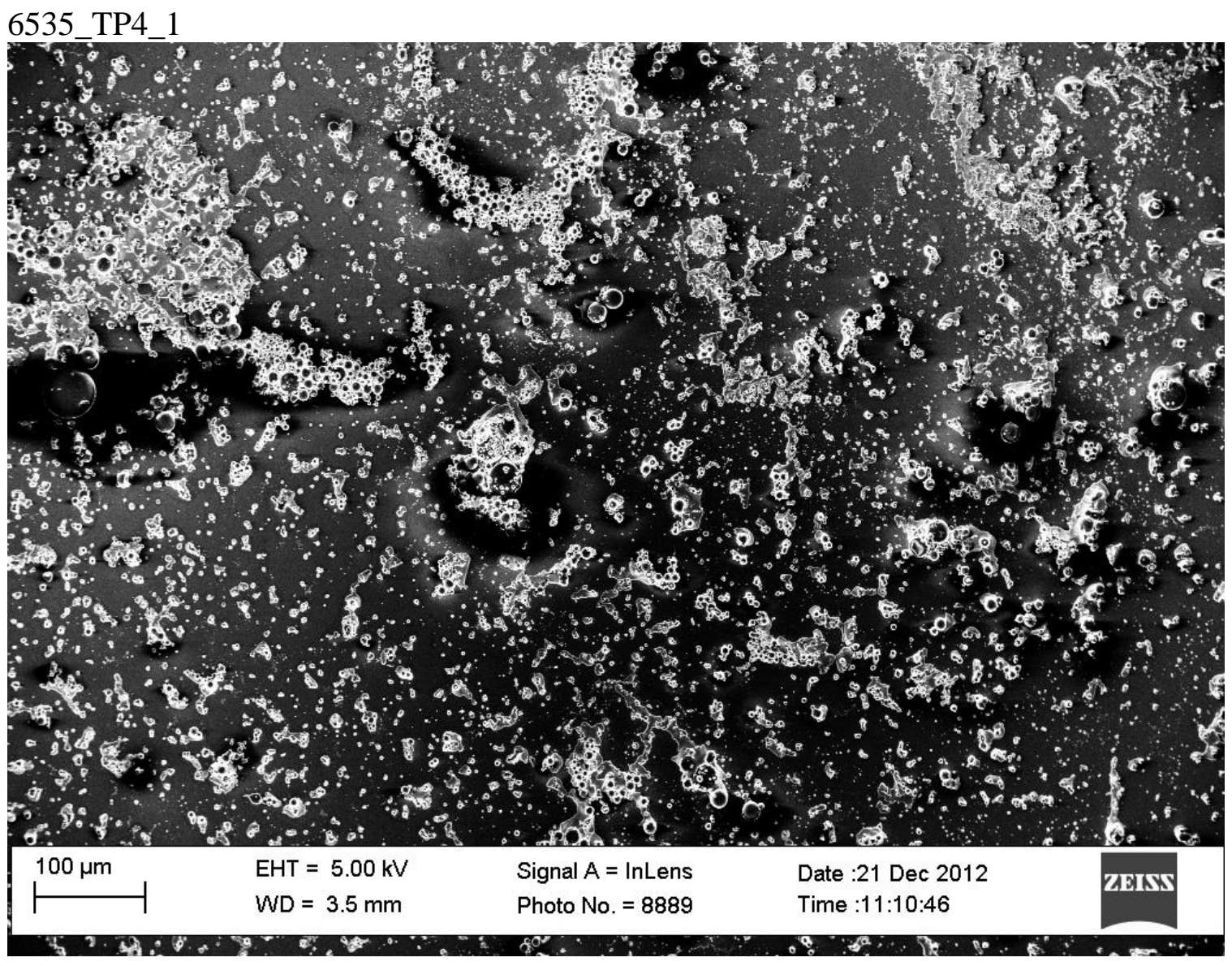




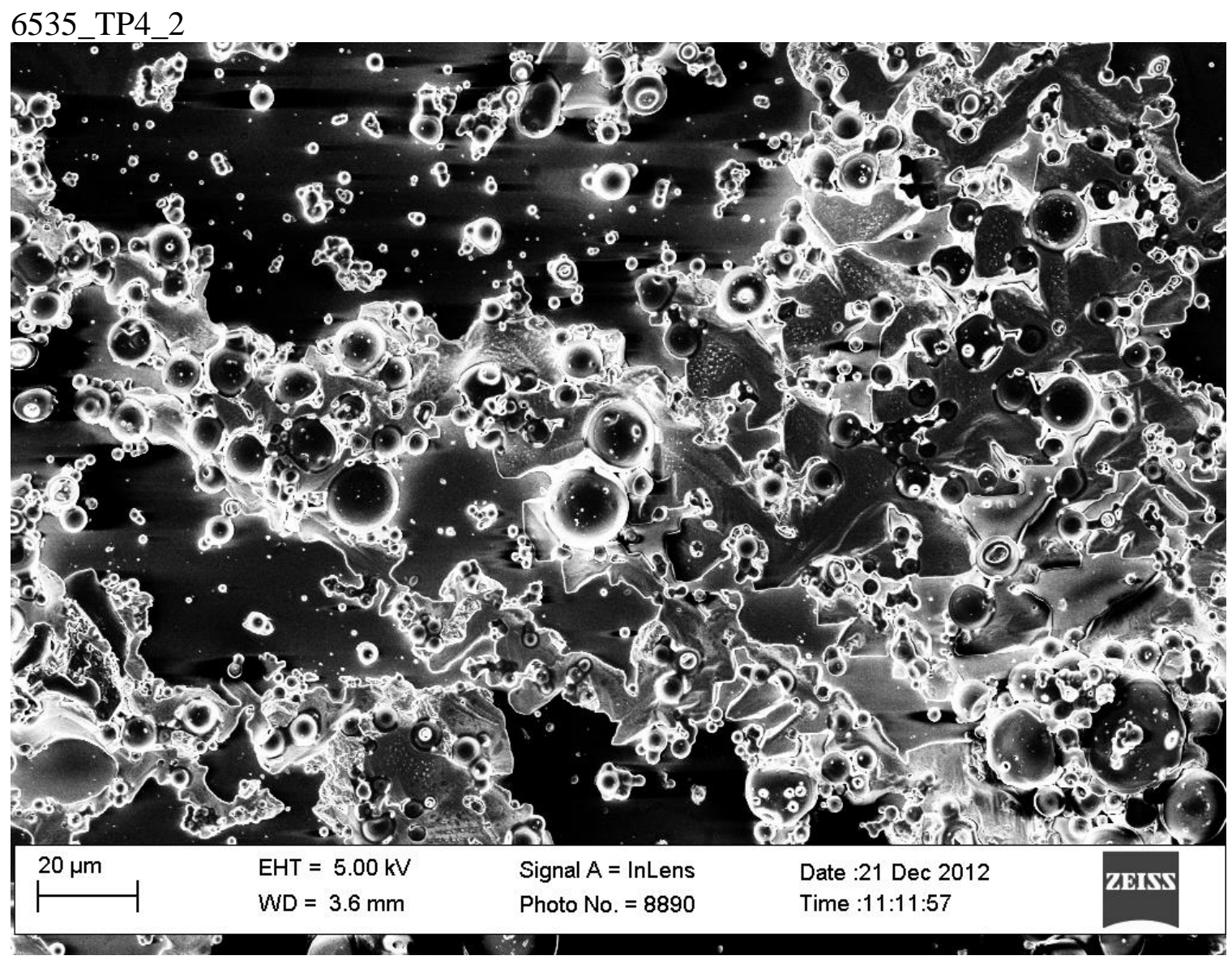




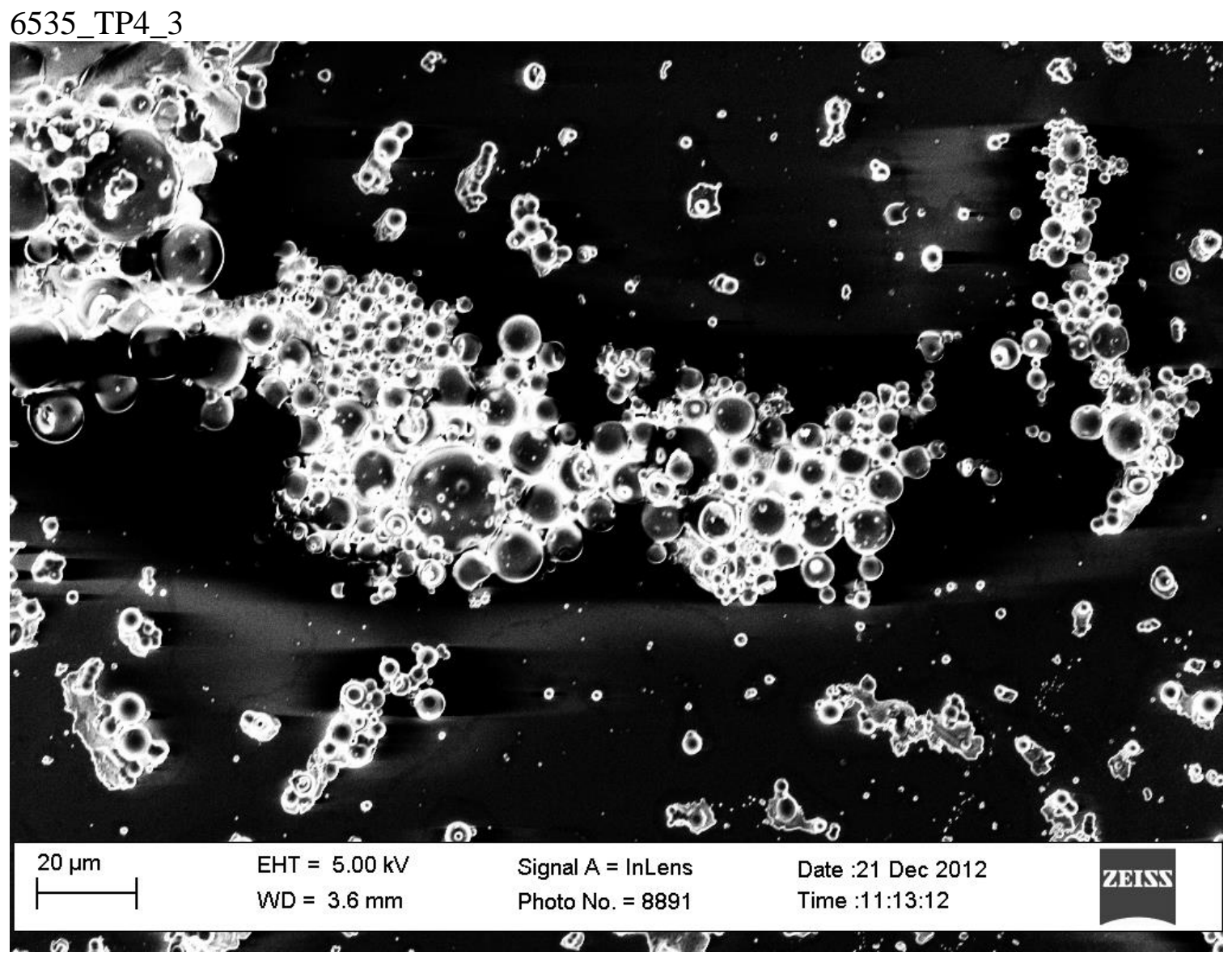




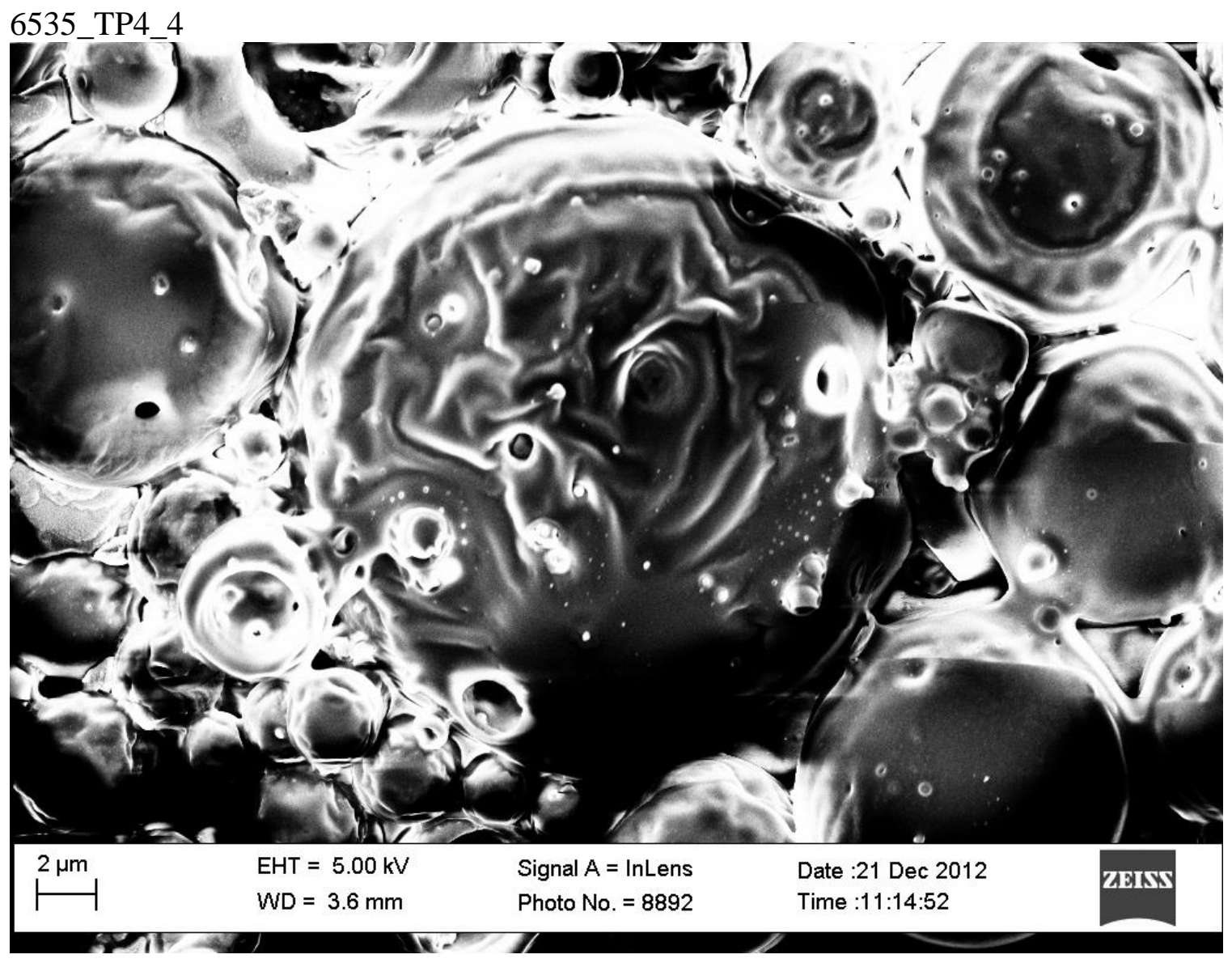




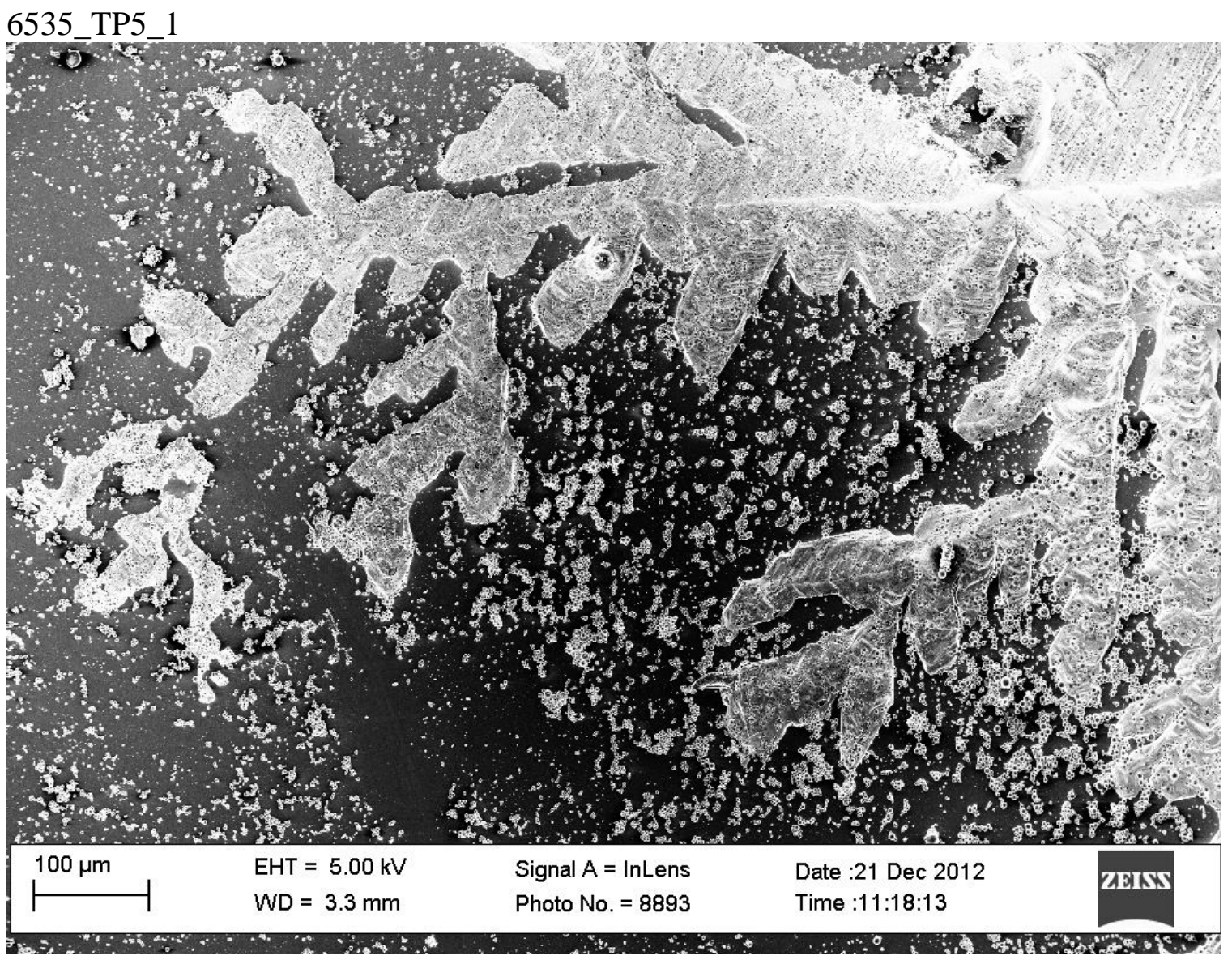




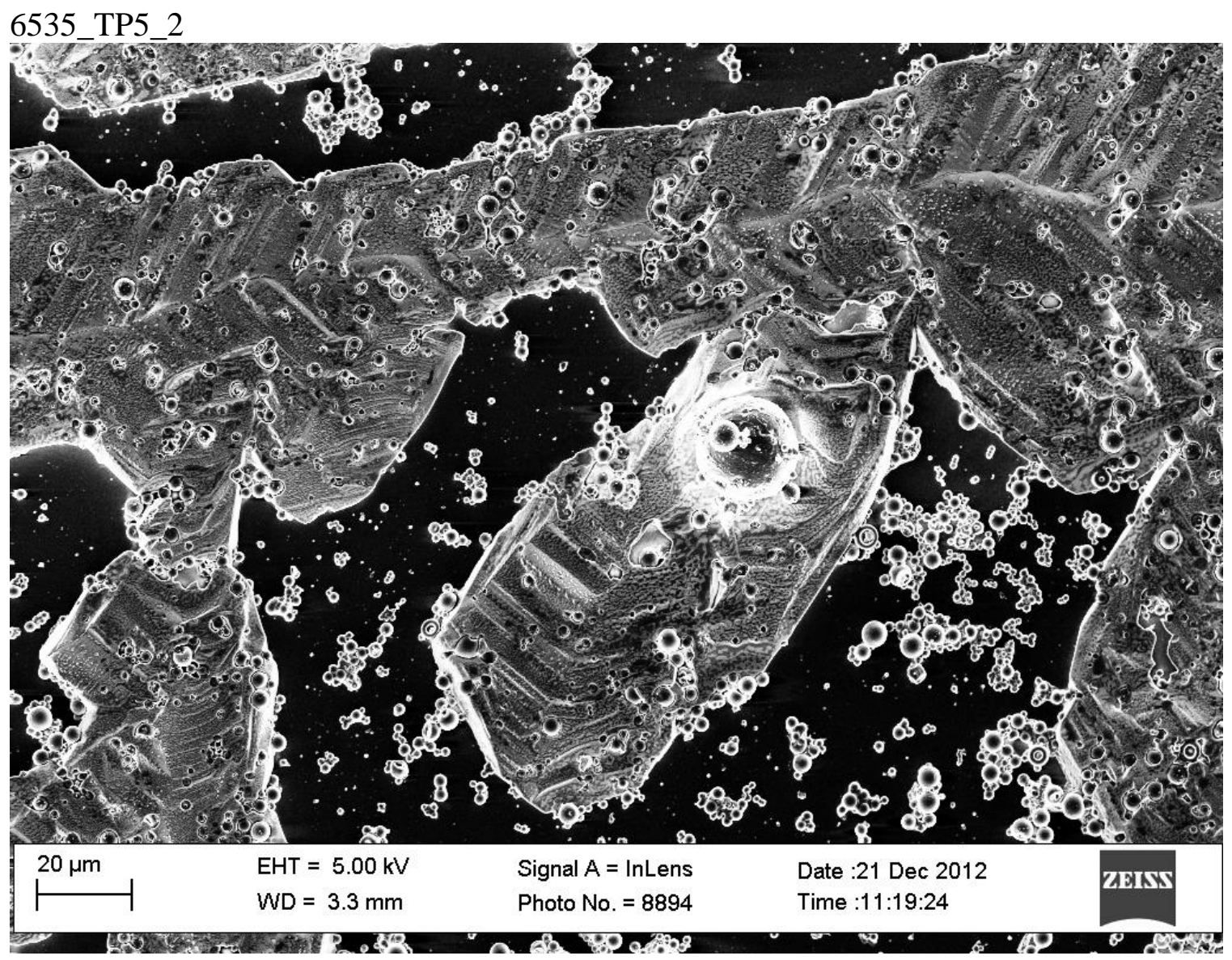




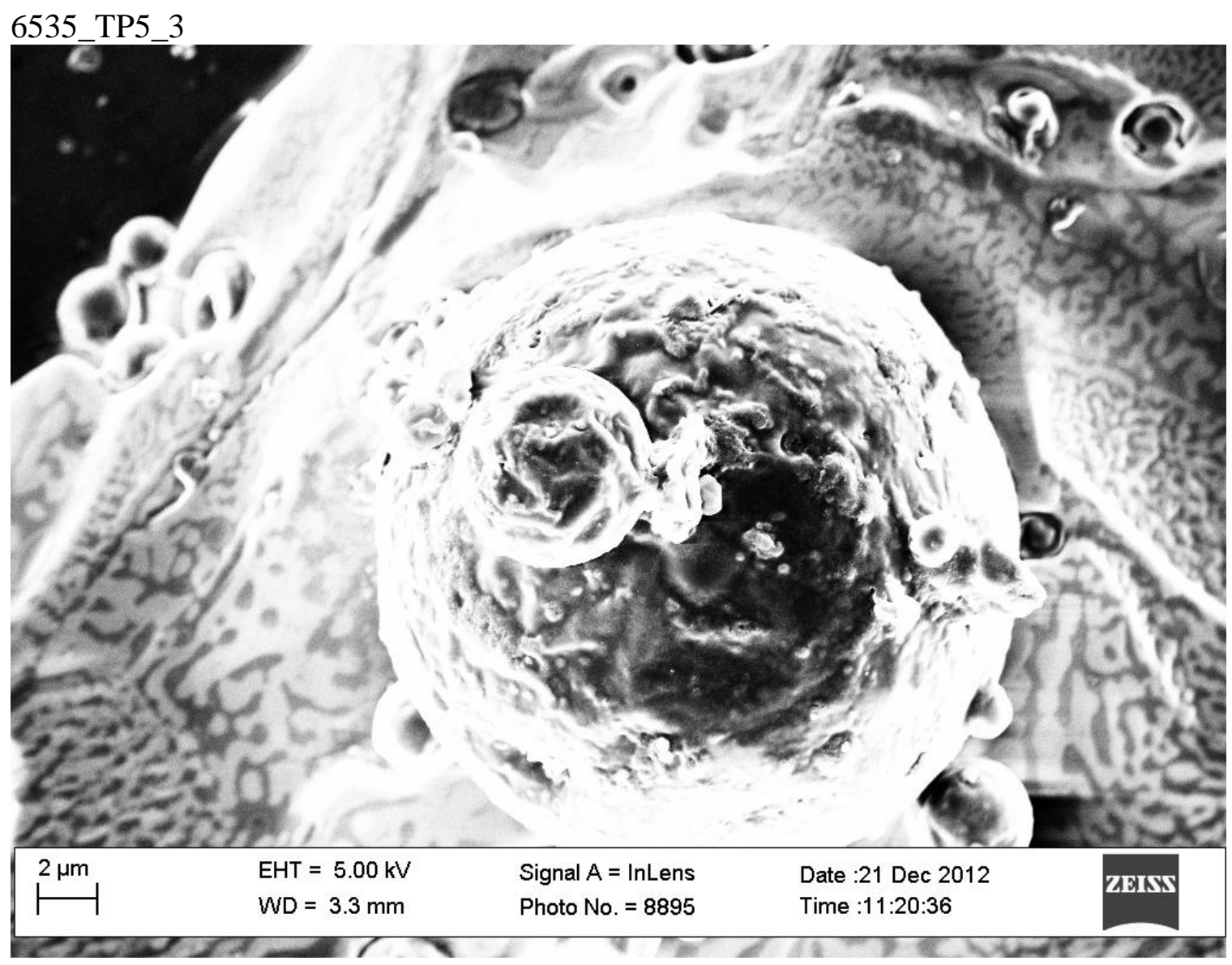




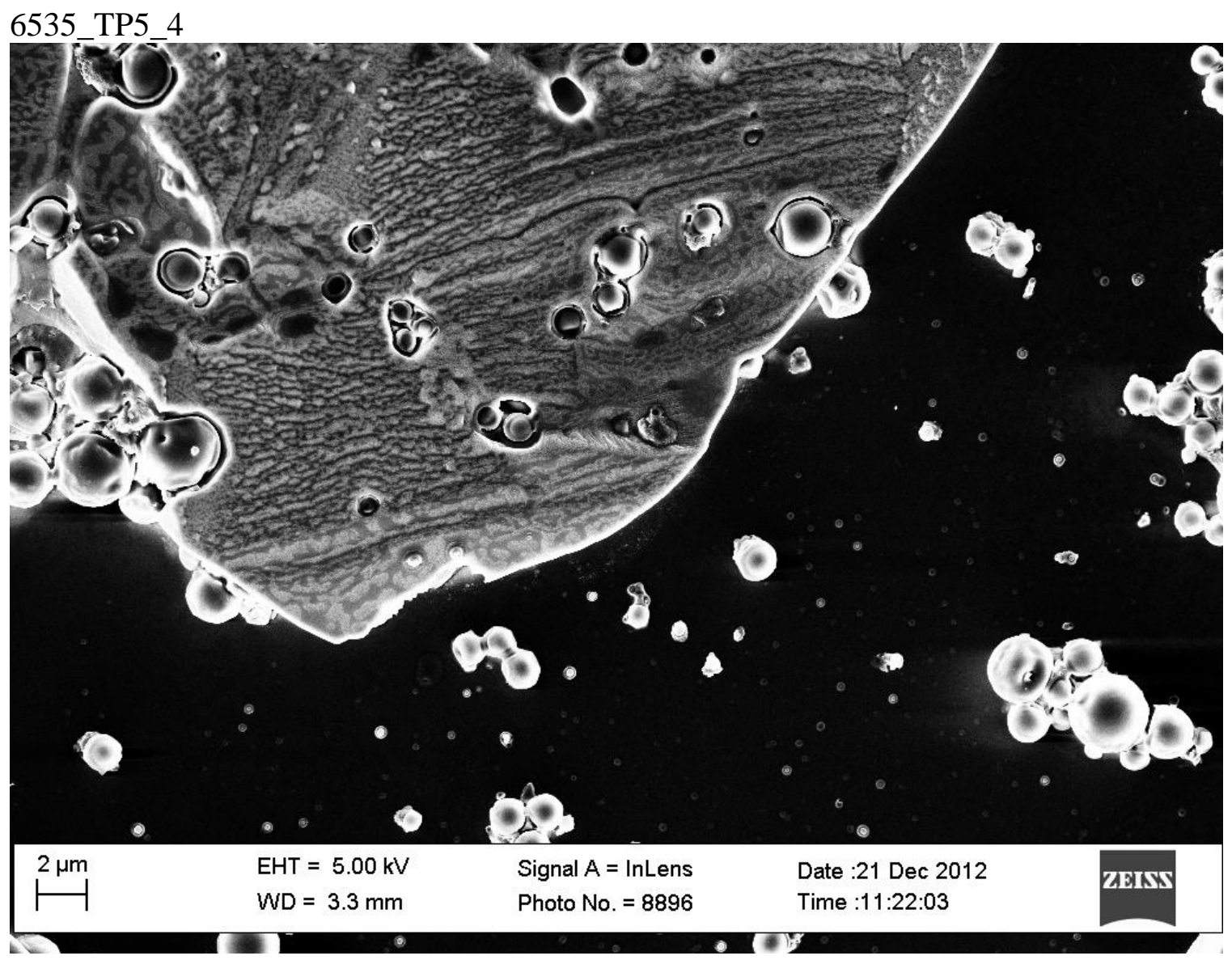




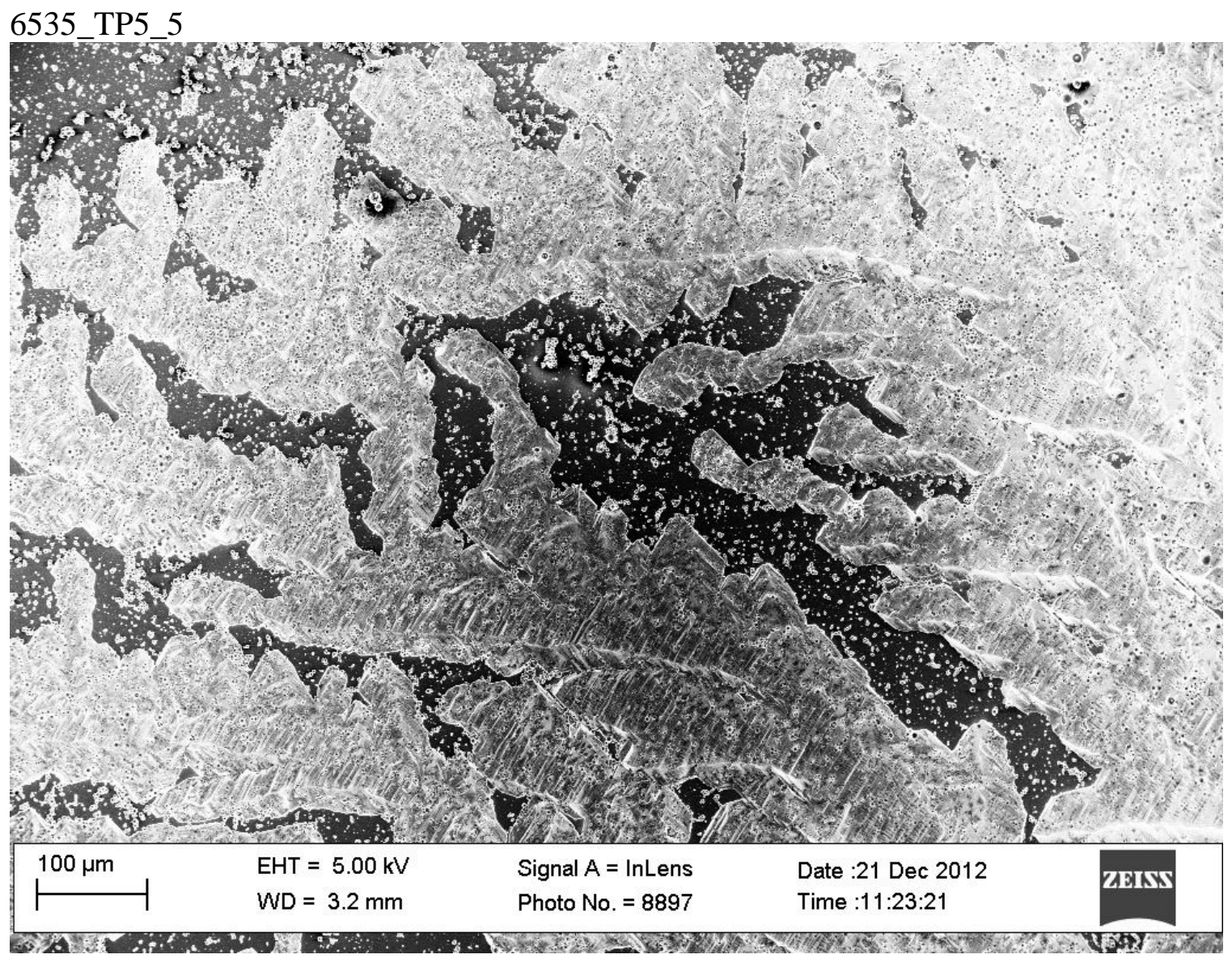




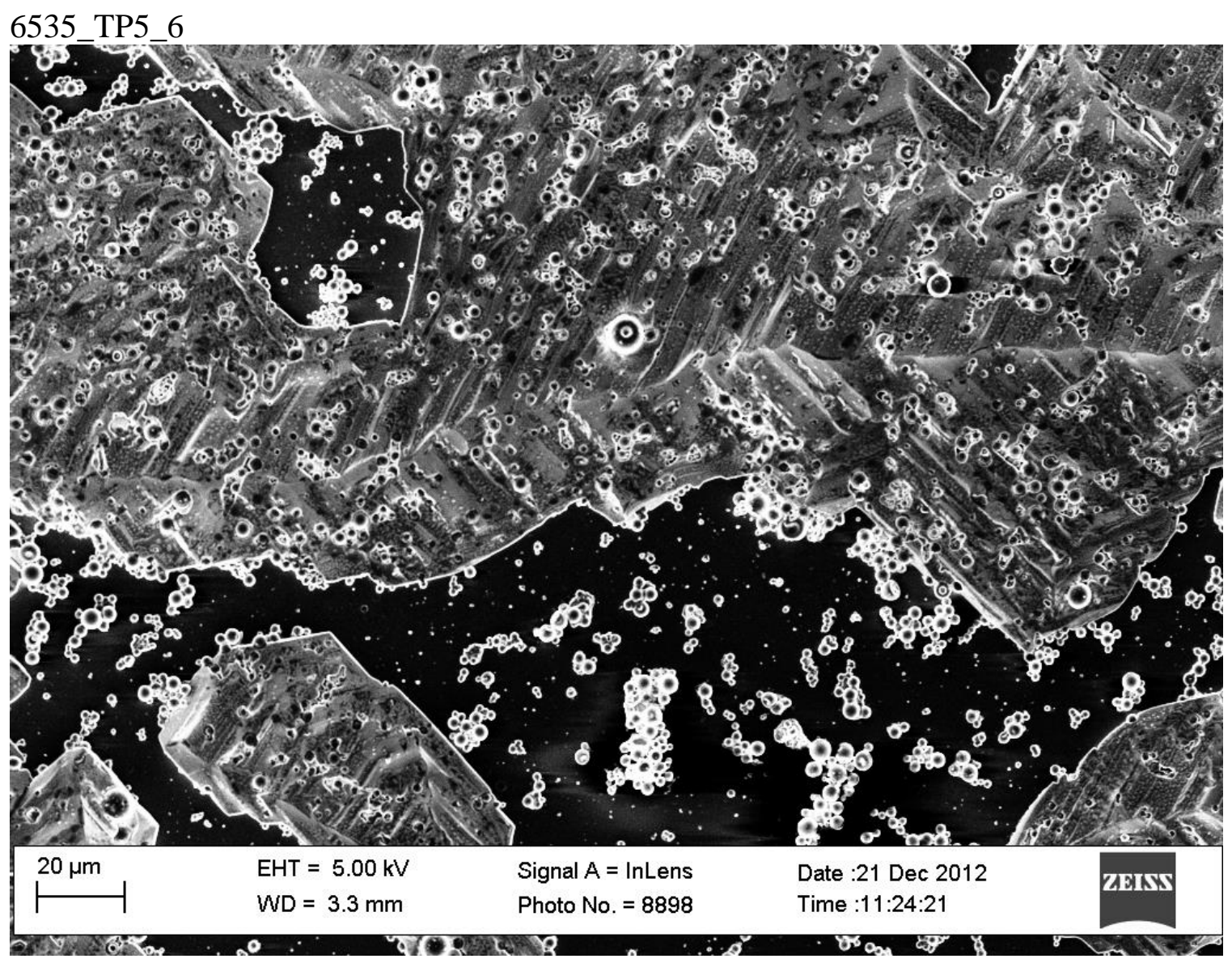




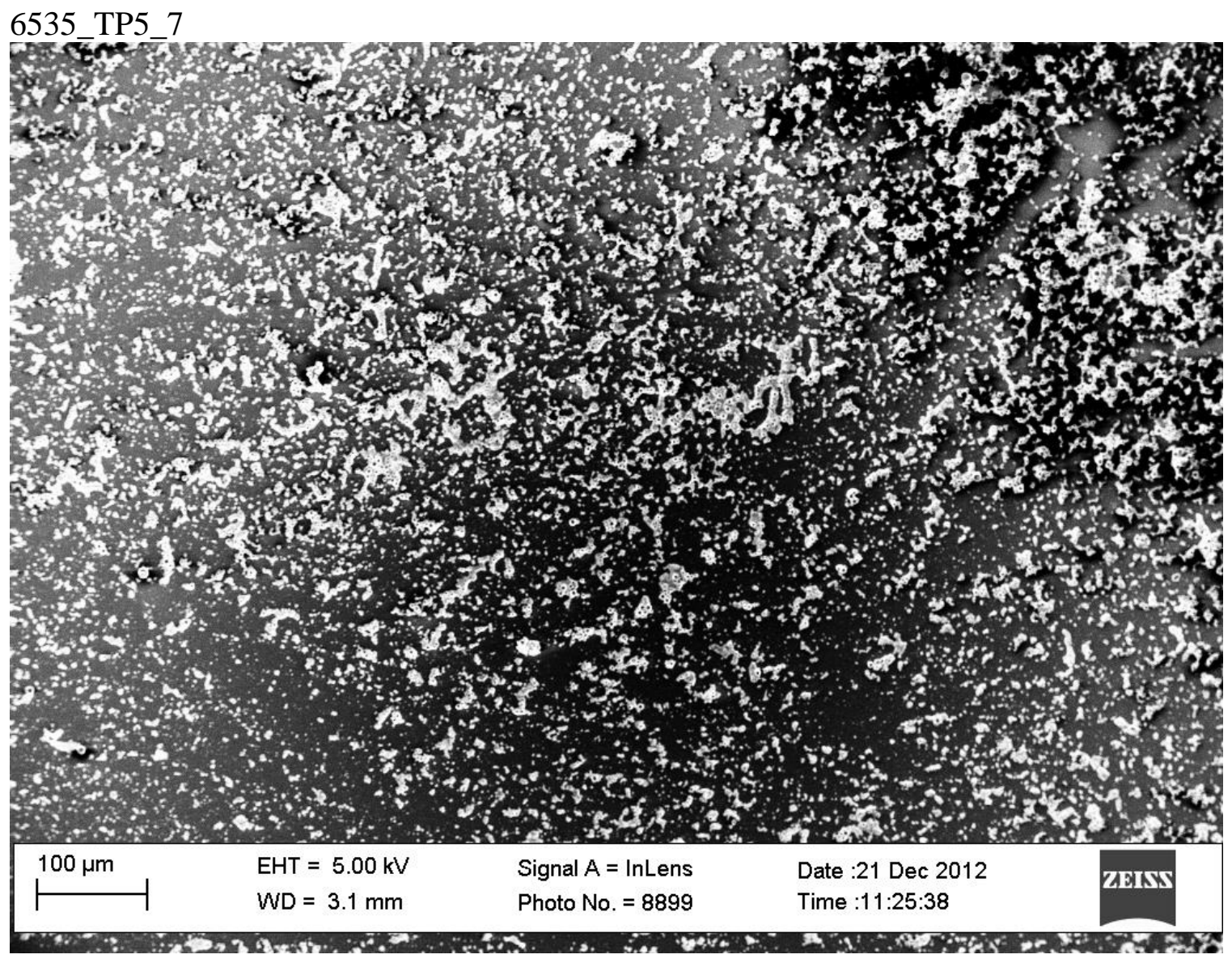




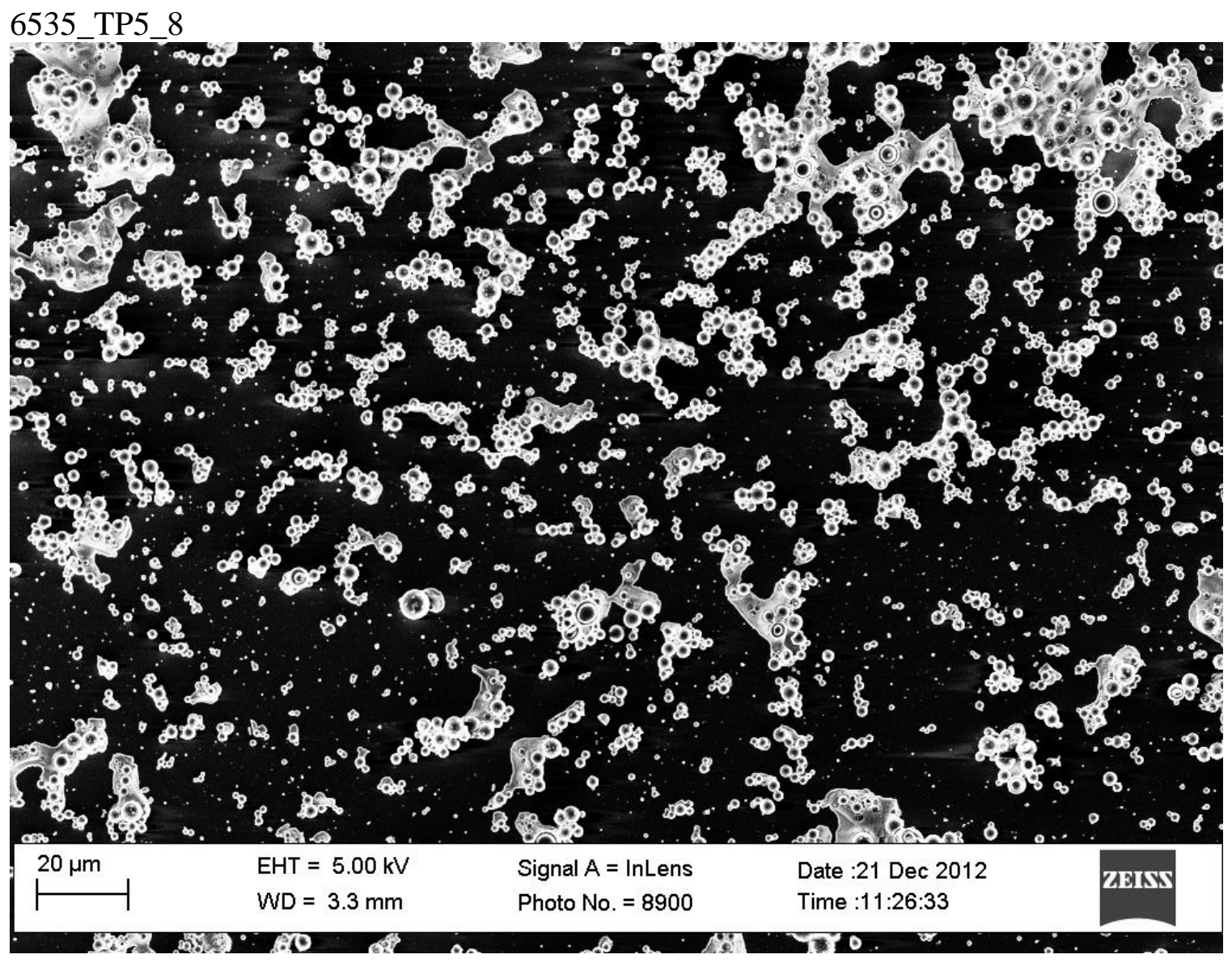




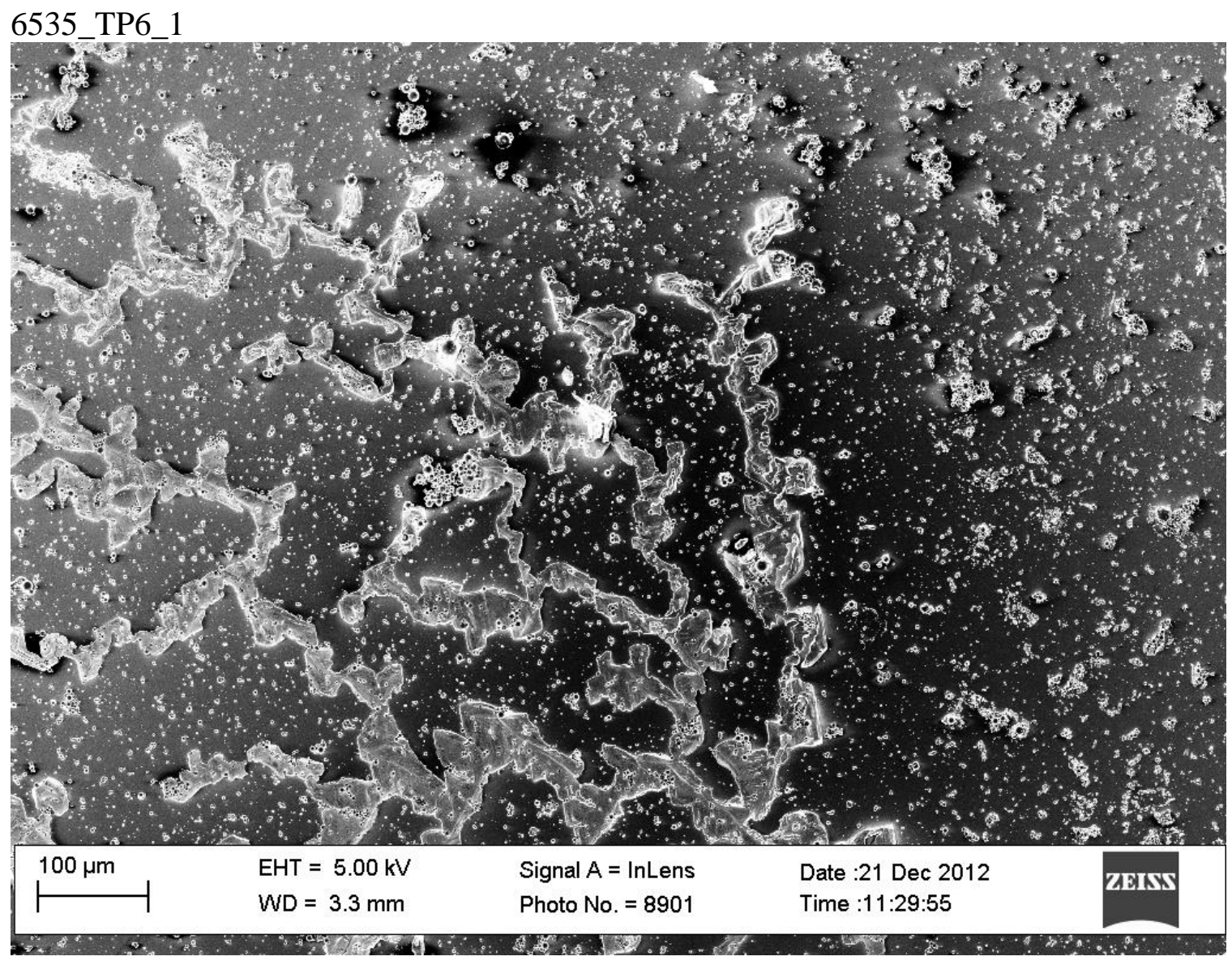




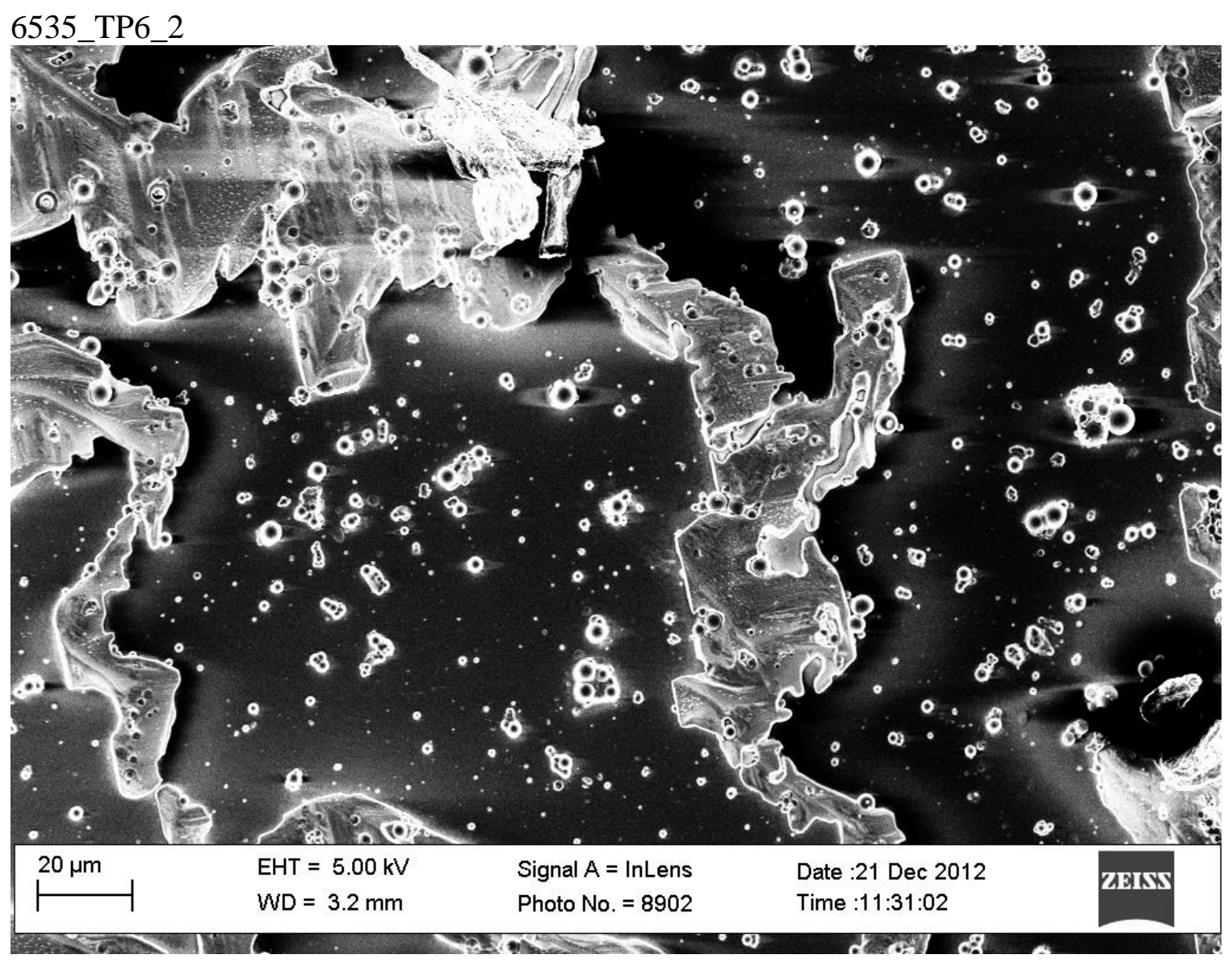




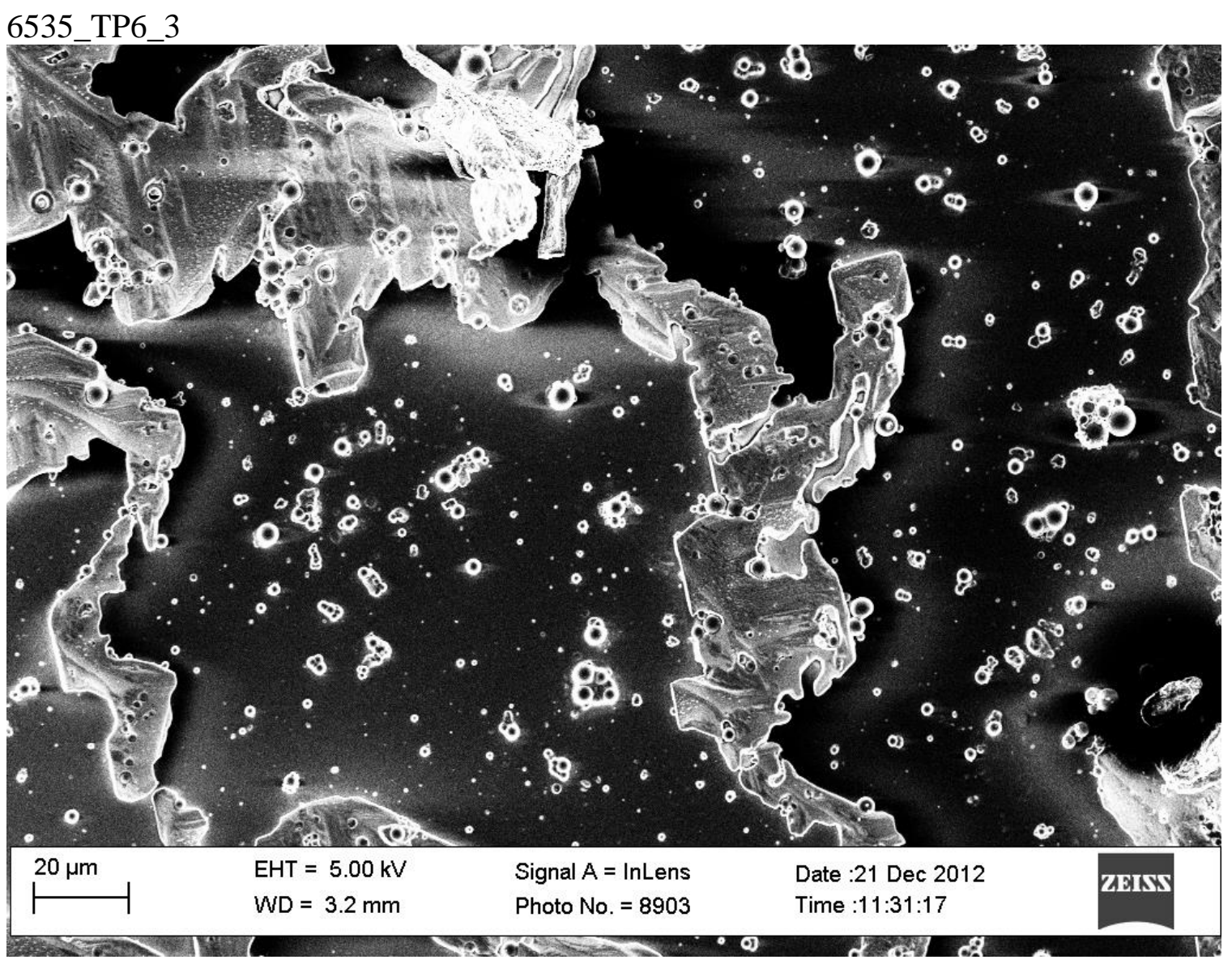




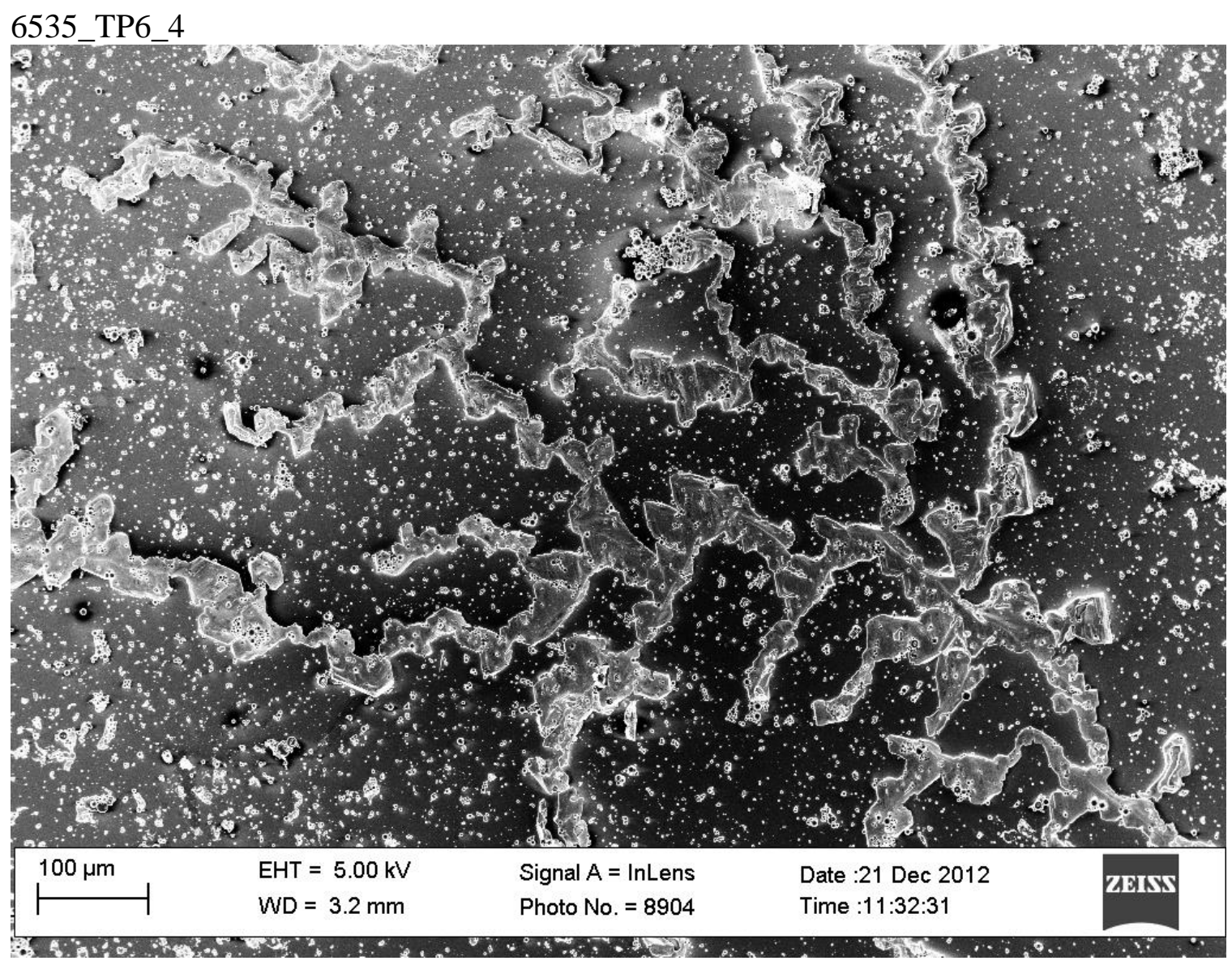




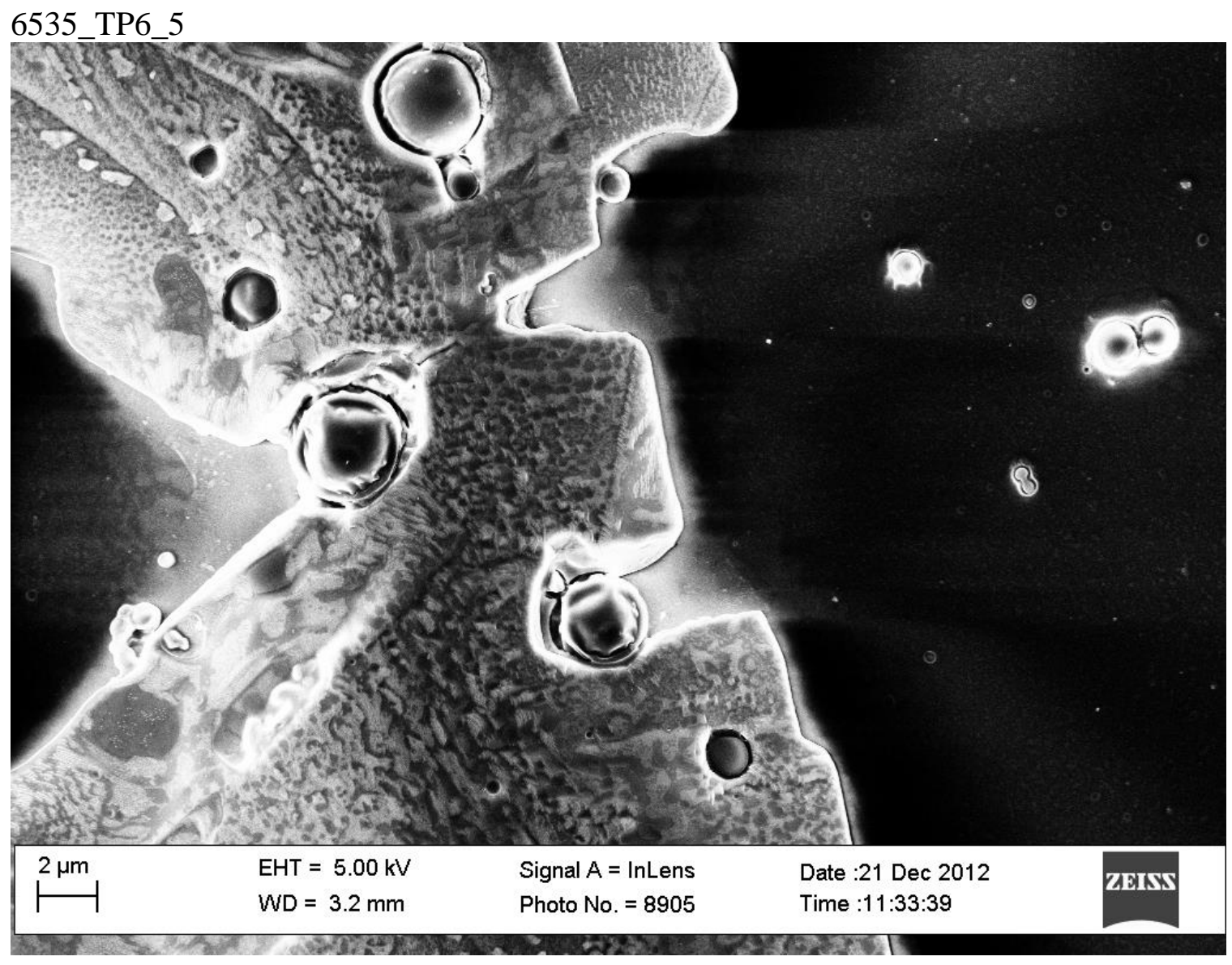




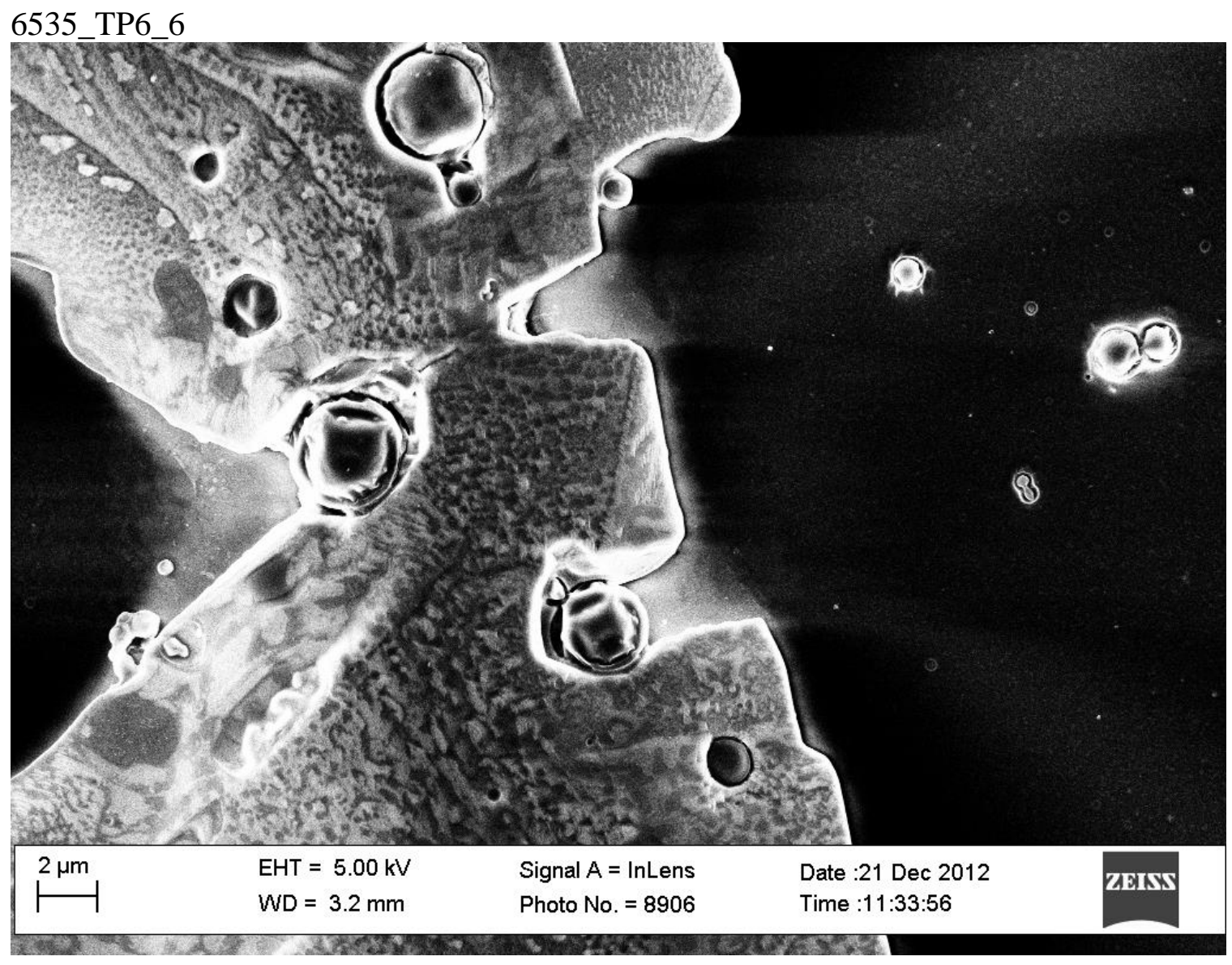

